AUTOMATED DETECTION OF AMPEROMETRIC SPIKES RESULTING FROM

QUANTAL EXOCYTOSIS AND ESTIMATION OF SPIKE AND PRE-SPIKE

FOOT SIGNAL PARAMETERS

\begin{tabular}{c} 
A Dissertation \\
presented to \\
the Faculty of the Graduate School \\
at the University of Missouri-Columbia \\
In Partial Fulfillment \\
of the Requirements for the Degree \\
Doctor of Philosophy \\
\hline SUPRYA BALAJI RAMACHANDRAN \\
Dr. Kevin D. Gillis, Dissertation Supervisor \\
MAY 2018
\end{tabular}


The undersigned, appointed by the dean of the Graduate School, have examined the dissertation entitled

\section{AUTOMATED DETECTION OF AMPEROMETRIC SPIKES RESULTING FROM QUANTAL EXOCYTOSIS AND ESTIMATION OF SPIKE AND PRE-SPIKE FOOT SIGNAL PARAMETERS}

presented by Supriya Balaji Ramachandran, a candidate for the degree of Doctor of Philosophy and hereby certify that, in their opinion, it is worthy of acceptance.

Kevin D. Gillis, D. Sc., Department of Bioengineering

Gary Yao, PhD, Department of Bioengineering

Liqun (Andrew) Gu, PhD, Department of Bioengineering

Lorin Milescu, PhD, Division of Biological Sciences 


\section{ACKNOWLEDGEMENT}

Several people contributed to this work. First, I thank my dissertation advisor Dr. Kevin D. Gillis for providing the opportunity to tackle this research problem. He is a polymath in his own right, with expertise in fields ranging from engineering, mathematics and physiology to scientific communication, management and leadership. There is much to be learnt from him and I consider myself incredibly lucky to be his student.

Second, I thank my dissertation committee members, Dr. Liqun (Andrew) Gu for offering his expert outlook on trends in biomedical research, Dr. Gary Yao for brainstorming methods to choose a research path and Dr. Lorin Milescu for his inputs on my algorithms and sharing methodologies from his forte in neuroscience.

Third, I thank our lab folks, Dr. Xin (Alice) Liu for generously sharing her know-how in amperometry, Dr. Andrew Winslow for his advice on signalprocessing, Syed Mehdi Orouji (Exocytronics LLC) for his handy tips on photolithography and manual-analysis of foot-signals. 


\section{TABLE OF CONTENTS}

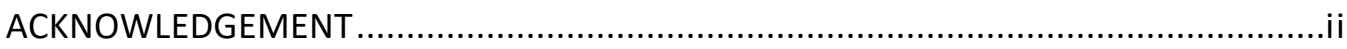

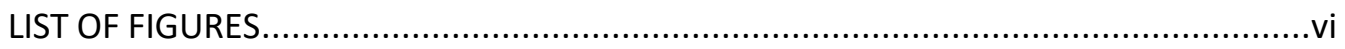

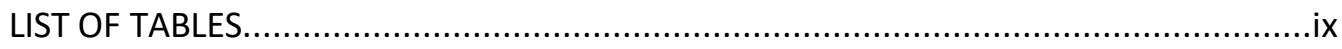

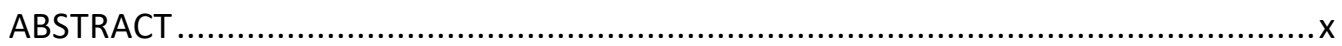

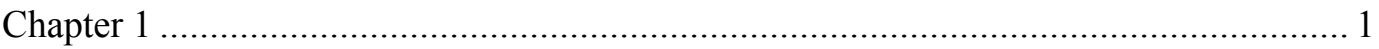

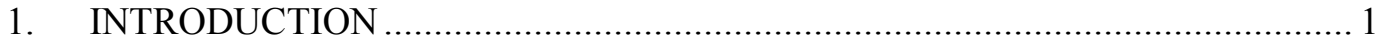

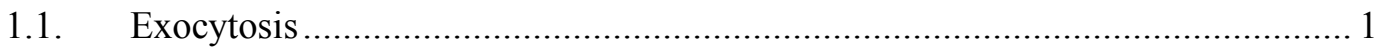

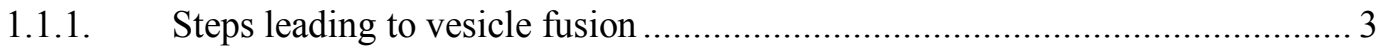

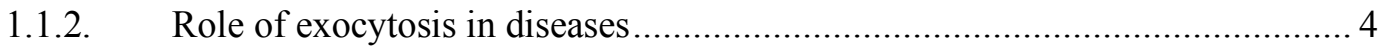

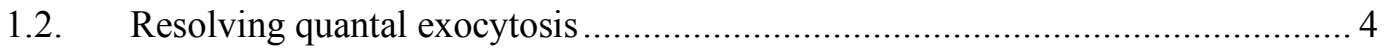

1.2.1. Electrochemical detection using amperometric measurements...................... 5

1.2.2. Temporal dynamics of a single-cell exocytotic event ................................... 6

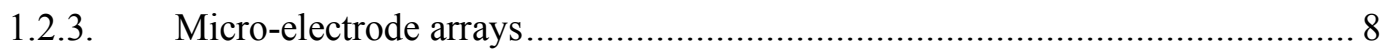

1.2.3.1. Limitations of Carbon Fiber amperometry ................................................ 8

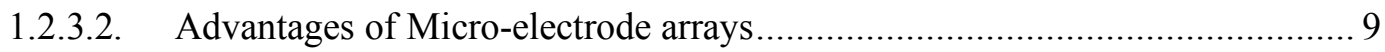

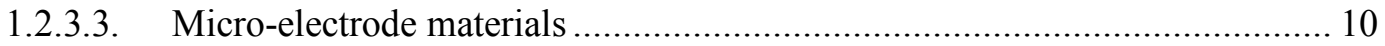

1.2.4. Different modes of fusion resolved in amperometry .................................. 12

1.2.5. Stages of fusion pore resolved in amperometry ….................................... 13

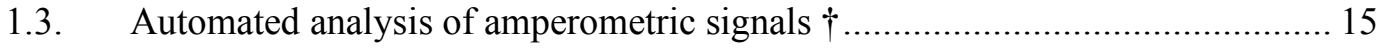

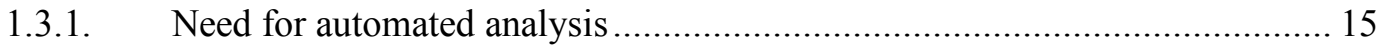

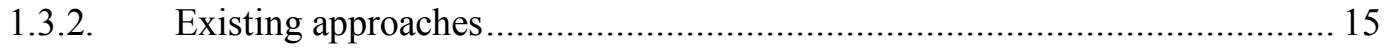

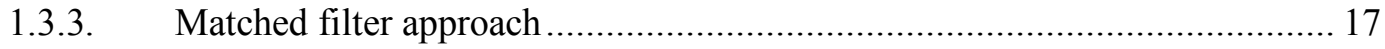

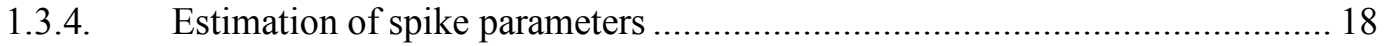

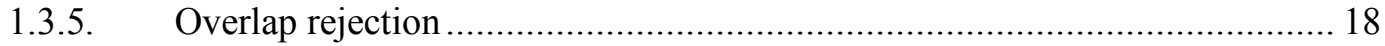

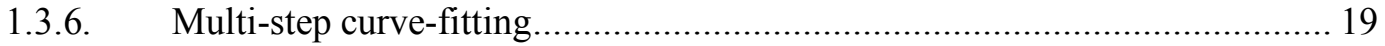

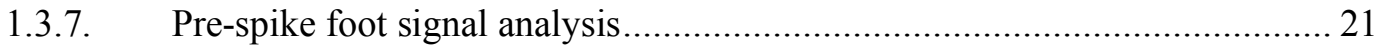

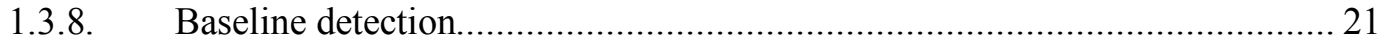

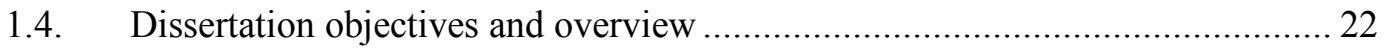

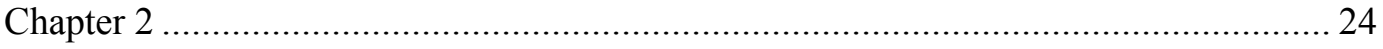

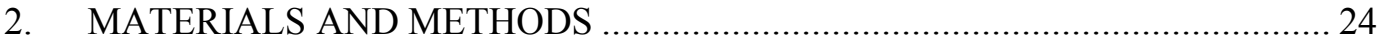

2.1. Fabrication of micro-electrode arrays ...................................................... 24

2.1.1. Electrode array Fabrication steps …..................................................... 24

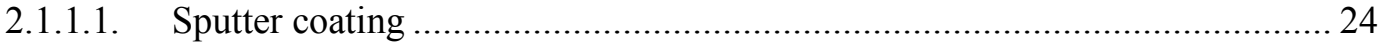




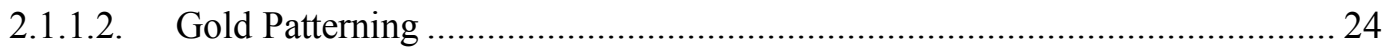

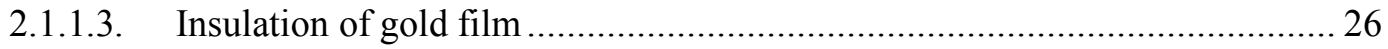

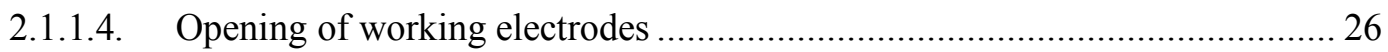

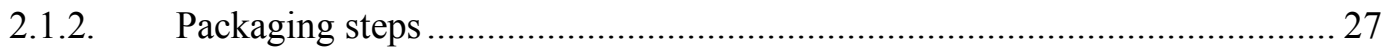

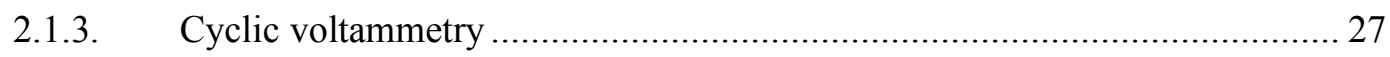

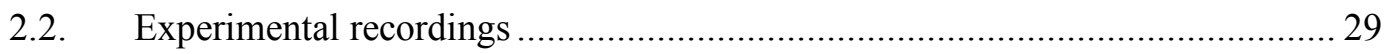

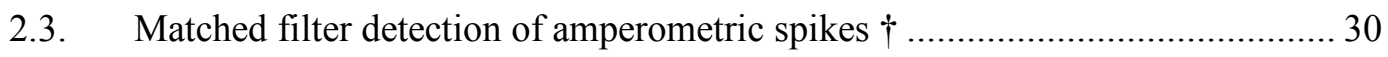

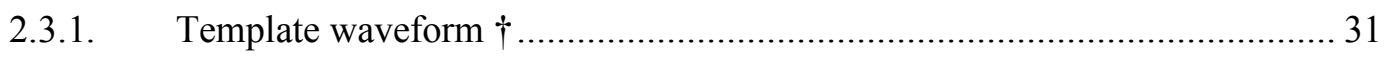

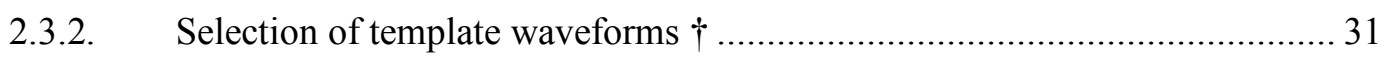

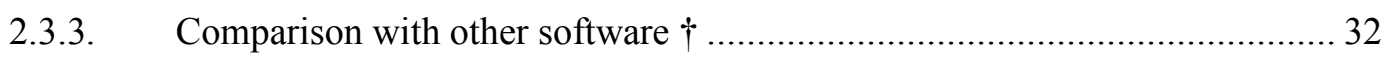

2.3.4. Quantification of detection performance using receiver operating characteristic plots $\dagger \quad 33$

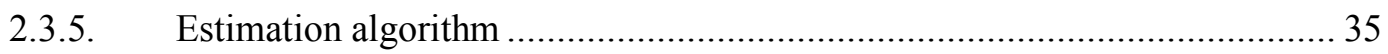

2.3.6. Rejection of overlapping spikes using EMF template parameters ................ 37

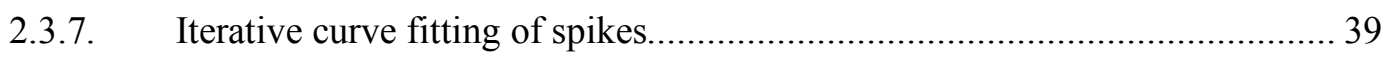

2.3.8. Baseline identification and pre-spike foot analysis .................................. 43

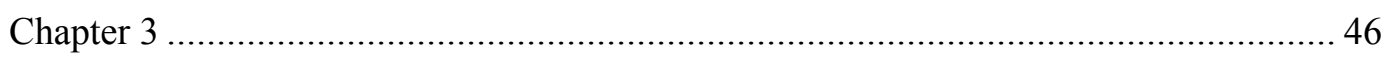

3. A MATCHED-FILTER ALGORITHM TO DETECT AMPEROMETRIC SPIKES

RESULTING FROM QUANTAL SECRETION † .................................................. 46

3.1. Automated detection of amperometric spikes .............................................. 46

3.1.1. Multiple templates are required to detect exocytotic spikes with varying time courses 47

3.1.2. Use of two thresholds reduces false-positives........................................ 49

3.1.3. The matched-filter approach is not very sensitive to filtering and can detect

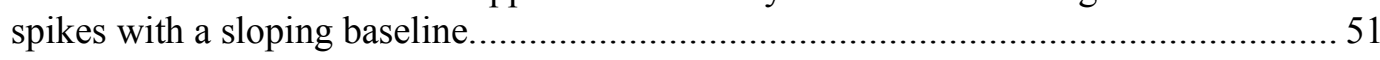

3.1.4. The matched-filter approach detects amperometric spikes with pre-spike

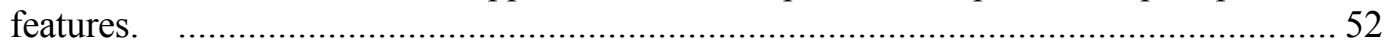

3.1.5. Extending the matched-filtering approach to detect spikes with a sum of two templates 54

3.1.6. The matched-filter approach outperforms the derivative-threshold approach .... 56

3.1.7. The matched-filter approach outperforms commercial event-detection software 58

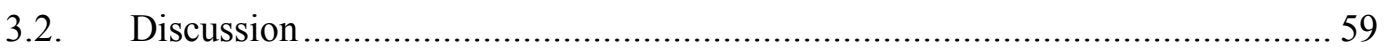

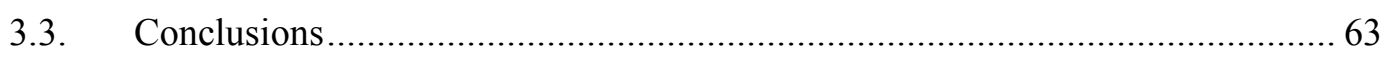

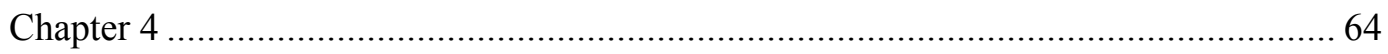


4. ESTIMATING AMPEROMETRIC SPIKE PARAMETERS RESULTING FROM QUANTAL EXOCYTOSIS USING CURVE FITTING SEEDED BY A MATCHEDFILTER ALGORITHM 64

4.1. Need for spike and pre-spike foot signal estimation..................................... 64

4.2. A two-criterion approach based on template fits successfully determines if

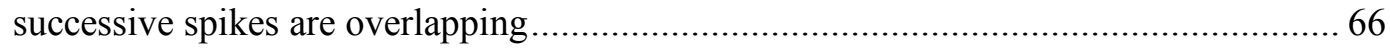

4.3. Matched filtering template parameters can reliably seed an iterative curve fit of

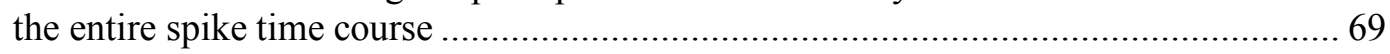

4.4. Iterative curve fits provide accurate parameters for computing Q, Imax and t50 ... 69

4.5. Use of the criterion score from matched filtering can accurately identify pre-

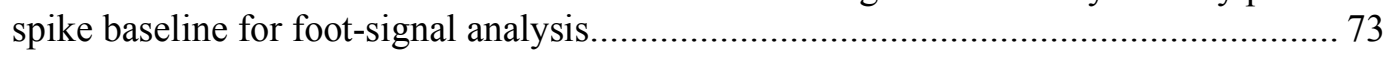

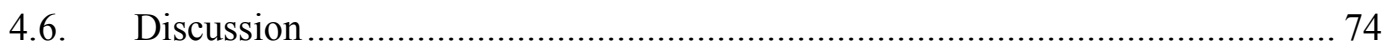

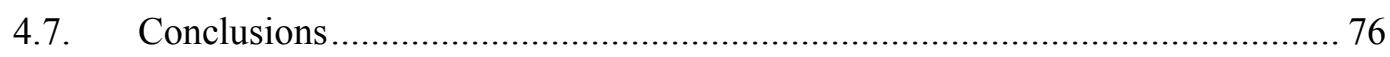

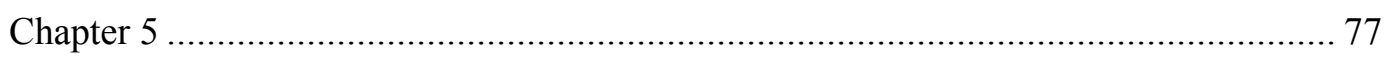

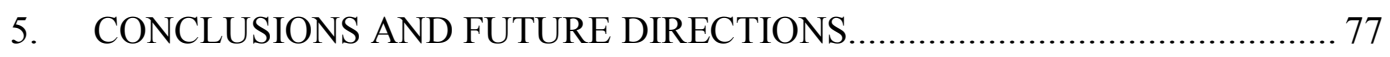

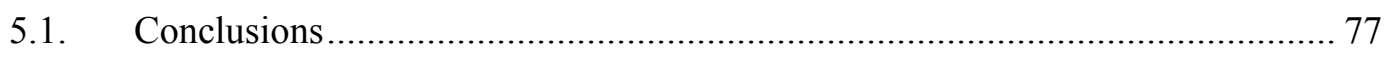

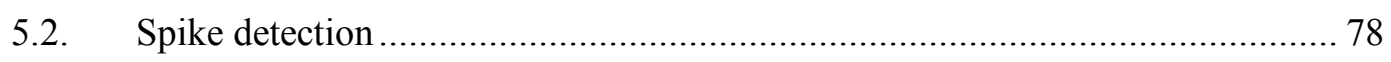

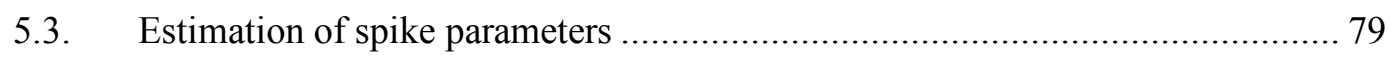

5.4. Detection and estimation of pre-spike foot signals................................... 80

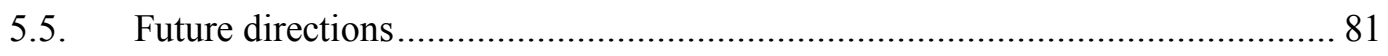

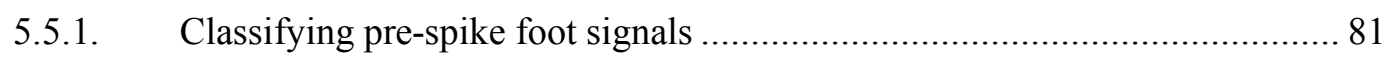

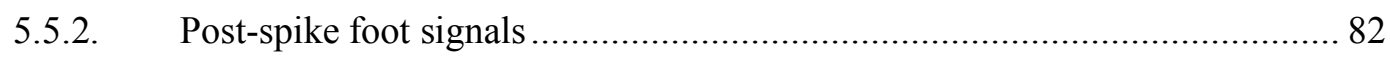

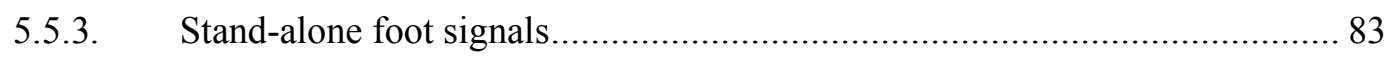

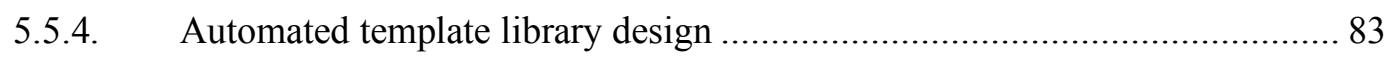

5.5.5. DT-MF hybrid spike detection approach............................................. 84

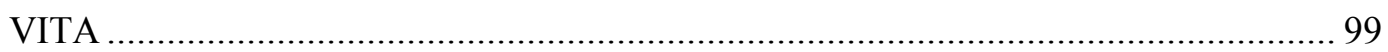




\section{LIST OF FIGURES}

Fig Page

Fig. 1.1. Cargo laden vesicles at two different stages of fusion at the cell membrane

Fig. 1.2. Experimental set-up of carbon fiber amperometry ............................... 5

Fig. 1.3. Three distinct phases in an exocytotic event...................................... 7

Fig. 1.4. Three distinct modes of fusion, full-fusion, kiss-and-run and kiss-andstay

Fig. 1.5. Different stages of fusion and corresponding amperometric trace (from

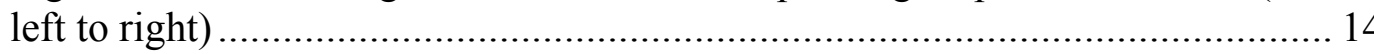

Fig. 1.6. Detection criterion for mini-synaptic events....................................... 16

Fig. 1.7. A typical amperometric trace preceded by a pre-spike foot signal ....... 17

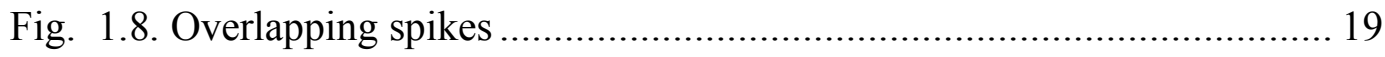

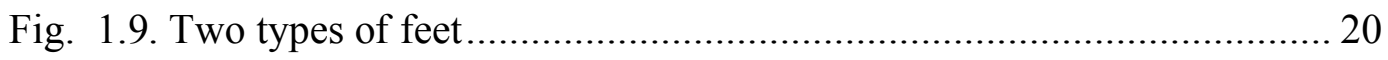

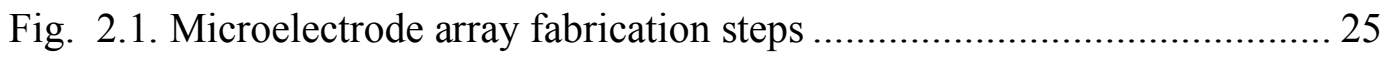

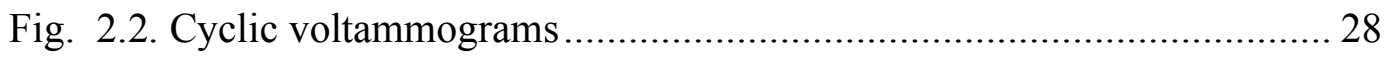

Fig. 2.3. Amperometric trace obtained from an electrode................................. 29

Fig. 2.4. Least squares can fit an amperometric spike with a template function and a receiving operator characteristic plot can quantify the performance of detection algorithms 
Fig. 2.5. Spike and pre-spike estimation algorithm.

Fig. 2.6. Overlapping spikes rejection criteria 38

Fig. 2.7. EMF template parameters provide seed values for a two-step non-linear curve fitting routine. 40

Fig. 2.8. Mf criterion scores can be used to determine baseline 44

Fig. 3.1. The heterogeneity of spike time courses necessitates the use of multiple templates for detection

Fig. 3.2. Filtering and baseline drift do not affect detection performance

Fig. 3.3. A two-threshold approach reduces false positives

Fig. 3.4. Foot signals do not affect detection of spikes

Fig. 3.5. The MF algorithm outperforms the DT approach in bovine chromaffin cell recordings

Fig. 3.6. The MF algorithm outperforms the DT approach in bovine chromaffin cell recordings

Fig. 3.7. Mf outperforms clampfit and minianalysis

Fig. 4.1. A two-criteria approach successfully rejects overlapping spikes for parameter analyses

Fig. 4.2. A sequential fitting process results in excellent fits of equation 12 to well-separated spikes

Fig. 4.4. Means of (a) Q, (b) $\mathrm{I}_{\max }$ and (c) $\mathrm{t}_{50}$ calculated using MF, Manual and $\mathrm{M} / \mathrm{S}$ (mosharov and sulzer, 2005) methods for amperometric recordings from mouse chromaffin cells. 
Fig. 4.3. Means of (a) Q, (b) $I_{\max }$ and (c) $t_{50}$ calculated using MF, Manual and $\mathrm{M} / \mathrm{S}$ (mosharov and sulzer, 2005) methods for amperometric recordings from bovine chromaffin cells ......................................................................... 70

Fig. 4.5. Histograms of spike parameters indicate the excellent performance of the new MF parameter-estimation algorithm................................................. 71

Fig. 4.6. The matched-filter based algorithm can accurately measure pre-spike

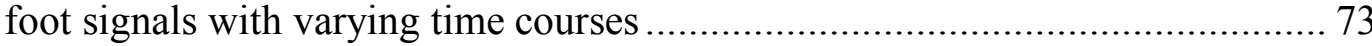

Fig. 4.7. Foot parameters of charge $\left(\mathrm{Q}_{\text {foot }}\right)$ and duration $\left(\mathrm{t}_{\text {foot }}\right)$ are shown as calculated by manual, $\mathrm{mf}$ and $\mathrm{m} / \mathrm{s}$ methods

Fig. 5.1. Classifying foot signals

Fig. 5.2. Post-spike foot signal 82

Fig. 5.3. Stand-alone-foot (SAF). 83 


\section{LIST OF TABLES}

Table

Page

Table 1.1. Comparison of four electrode materials for micro-electrode fabrication

Table 4.1. Medians of Q, Imax, and t50 computed for bovine and mouse chromaffin cell recordings using the new matched-filter based algorithm (MF), manual analysis, and the algorithm from (Mosharov and Sulzer, 2005) (M/S)... 71

Table 4.2. Comparison of centers $(\mu 1,2)$ and spreads $(\sigma 1,2)$ of bi-modal gaussian distributions

Table 4.3. Medians of foot parameters tfoot and Qfoot computed for bovine chromaffin cell recordings. 


\title{
AUTOMATED DETECTION OF AMPEROMETRIC SPIKES RESULTING FROM QUANTAL EXOCYTOSIS AND ESTIMATION OF SPIKE AND PRE-SPIKE FOOT SIGNAL PARAMETERS
}

\author{
Supriya Balaji Ramachandran
}

Dr. Kevin D. Gillis Dissertation Supervisor

\begin{abstract}
Electrochemical microelectrodes can detect single-vesicle release events as "spikes" of amperometric current. We developed a template based "matched-filter" approach that performs least squares fit of a library of templates to the data and identifies a spike when a detection criterion score given by the ratio of amplitude to the standard error exceeds a minimum threshold. This method outperformed existing approaches and detected $>95 \%$ of true spikes for a mere $2 \%$ false positive rate as evidenced by receiver operating characteristic plots of sensitivity vs specificity. The next step is estimation of spike parameters like peak amplitude (Imax), half-maximal width $\left(\mathrm{t}_{50}\right)$ and area under the curve (Q) which inform maximal flux, flux duration and charge respectively. Closely successive overlapping spikes are ambiguous to estimate as they may not decay back to baseline and should be rejected. Matched filter approach not only provided robust spike detection but also parameter seed values to reject overlapping spikes and also perform iterative curve fitting of spikes. The remaining well-separated spikes were iteratively fit in two phases, first by fitting rising and decaying phases separately and second by fitting the entire time course using seed values from the matched filter template parameters. Using curve-fit parameters, $I_{\max }, t_{50}$ and $Q$ were calculated. Histograms of these parameters had bi-modal Gaussian distributions with centers and spreads within $12 \%$ and $4 \%$ of histograms created using manually analyzed data. The pre-spike baseline was estimated using a novel application of the matched-filter criterion scores and the estimation of pre-spike foot signal parameters such as charge $\left(\mathrm{Q}_{\text {foot }}\right)$ and duration $\left(\mathrm{t}_{\text {foot }}\right)$ yielded means, and medians within $10 \%$ of manually computed parameters.
\end{abstract}




\section{Chapter 1}

\section{INTRODUCTION}

\subsection{Exocytosis}

Exocytosis is the fusion of intracellular vesicles with the plasma membrane, leading to extrusion of vesicle contents to the extracellular space. Vesicles are structures found within the cell and are composed of a lipid bilayer membrane enclosing cargo to be transported outside the cell. The extruded vesicular contents perform various vital functions. Cargo carried by these vesicles include

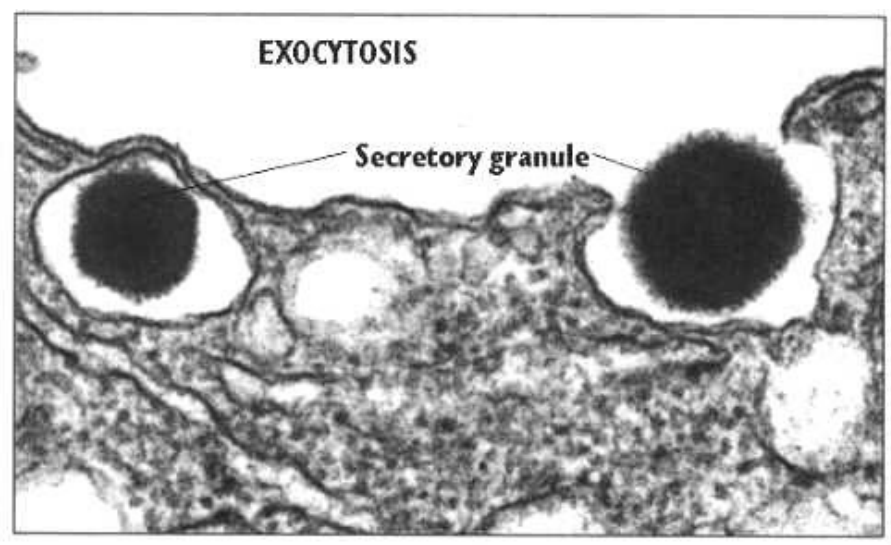

Fig. 1.1. Cargo laden vesicles at two different stages of fusion at the cell membrane

Reproduced from the "Molecular biology of the cell" ( $3^{\text {rd }}$ edition) (Alberts B) with copyright permission from John Wiley and sons.

neuropeptides (e.g., neuropeptide Y) (Thureson-Klein A Fau - Klein et al.), neurotransmitters (e.g., catecholamines such as dopamine, epinephrine, norepinephrine) (Biermann et al.), and hormones (e.g., vasopressin, oxytocin (Tobin et al., 2012), insulin (Jewell et al., 2010)) . Exocytosis is categorized into 
two types based on whether a stimulus is required to trigger the process (Alberts B, 2002). These types are (a) constitutive exocytosis - a stimulus-independent process that typically involves transport of proteins that are to be integrated with the plasma membrane and (b) regulated exocytosis - a calcium stimulusdependent process that involves release of hormones, neurotransmitters and cytokines among other small signaling molecules. The release of catecholamines from vesicles in adrenal chromaffin cells was evidenced through electron microscopy and biochemical analysis (Banks and Helle, 1965) of the released substances. In the following year, it was reported that catecholamines residing inside intracellular vesicles were extruded by a process called "reverse pinocytosis"(Kirshner et al., 1966), where pinocytosis (Doherty and McMahon, 2009 ) is the intake of extracellular fluid into the cell via vesicles. In 1963, De Duve (de Duve, 1963) coined the term "exocytosis" to describe the fusion mechanism. Early electron micrographs (Birks et al., 1960) did not strongly demonstrate the actual fusion of the vesicle with the cell membrane because there was no evidence of whether static images of vesicles represented vesicles exiting the cell or of new vesicles forming. Through intra-cellular micro-electrode recordings at the frog neuromuscular junction, of end plate potentials (EPP) elicited by acetylcholine release, and statistical analysis of EPP amplitudes, Bernard Katz (Fatt and Katz, 1952; Fatt and Katz, 1951) and colleagues concluded that the EPP consists of multiple quanta of cargo thereby formulating a "quantal hypothesis" of neurotransmitter release. It was later demonstrated through freeze fracture microscopy combined with 
electrophysiological recordings (Heuser et al., 1979) that vesicles indeed fuse with the cell membrane, releasing cargo in "quanta" or packets (Heuser et al., 1979). Such membrane fusion is facilitated via a complex mechanism of interaction between vesicular and membrane proteins.

\subsubsection{Steps leading to vesicle fusion}

During exocytosis, the vesicular bi-lipid membrane fuses with the plasma membrane, and then forms a fusion-pore, starting with a nanometer-scale (Chow et al., 1992a; de Toledo et al., 1993b) connection to the extra-cellular space, through which cargo is released. Furthermore, the fusion pore expands to rapidly release the vesicle contents. The entire fusion process at the release site occurs in a multi-step manner (Jahn and Fasshauer, 2012; Südhof, 1995), that is highly dependent on interactions between vesicle and membrane Soluble Nethylmaleimide-sensitive factor Attachment protein Receptor (SNARE) proteins (Davis et al., 1999; Zhou et al., 2015) as well as calcium sensitive (de Wit et al.; Man et al., 2015; Mohrmann et al., 2013) and chaperone proteins (Toonen and Verhage, 2007). The steps leading to release are: (a) docking - alignment and tethering of vesicle and plasma membranes in a fusion-ready state (Oheim et al., 1998; Rizo and Rosenmund, 2008; Verhage and Sørensen Jakob, 2008). (b) priming - priming makes the vesicle "fusion competent" (Liu et al., 2010; Voets et al.; Walter et al., 2013) in order to ensure that a calcium influx will lead to fast cargo release (Imig et al.; Jung et al., 2016). (c) fusion - a calcium binding vesicle-protein, synaptotagmin (Rizo et al.) regulates SNARE-dependent fusion of vesicle and plasma membranes and formation of a fusion pore, leading to the 
release of contained cargo. Release of the entire vesicle contents may occur, or,

alternatively, the fusion pore may re-close rapidly during endocytosis (Heuser and Reese, 1973). Modes of fusion are described in section 1.2.4.

\subsubsection{Role of exocytosis in diseases}

Early studies in pituitary adenomas reported the spatial misplacement of exocytosis sites (Horvath and Kovacs, 1974) in tumorous cells, hence potentiating diagnosis of the disease. Proteins implicated in exocytosis have been reported at lower levels in schizophrenics (Davidsson et al.). The drug Botox (Botulinum neurotoxin type-A) acts by inhibiting exocytosis and is in used clinically to treat numerous conditions (Aoki, 2004) such as gastrointestinal spasms (Brisinda et al., 2004) and post-stroke muscle over-activity (Esquenazi and Mayer) as well as cosmetic treatment of glabellar frown lines (Frampton and Easthope). Migraine prophylaxis has been attributed to drug induced blockade of exocytosis (RuizNuño et al., 2003). The role of a fusion protein, amisyn (Constable et al., 2005) has been recently attributed in the narrowing of fusion pore diameter, leading to reduced insulin secretion in Type II diabetics (Hastoy et al., 2017). Thus, the study of exocytosis offers the potential for advancing the medical treatment of various diseases.

\subsection{Resolving quantal exocytosis}

The fusion of a single vesicle and extrusion of its contents is referred to as an exocytotic event. Important features of an exocytotic event are (a) the time courses of membrane fusion and content release and (b) the amount (quanta) of cargo released during the release. Studying the variations in the aforementioned 
parameters upon perturbation of the cell with a pharmacological agent or genetic manipulation provides clues about how the drug or protein target affects exocytosis (Borges et al., 2006). Quantal secretion of electroactive transmitter from single vesicles can be resolved through electrochemical detection.

\subsubsection{Electrochemical detection using amperometric measurements}

Electro-chemical detection of transmitter molecules can be carried out using amperometric micro-electrodes (Chow; Chow et al., 1992a; Leszczyszyn et al.). A class of vesicle cargo called catecholamines (epinephrine, norepinephrine,

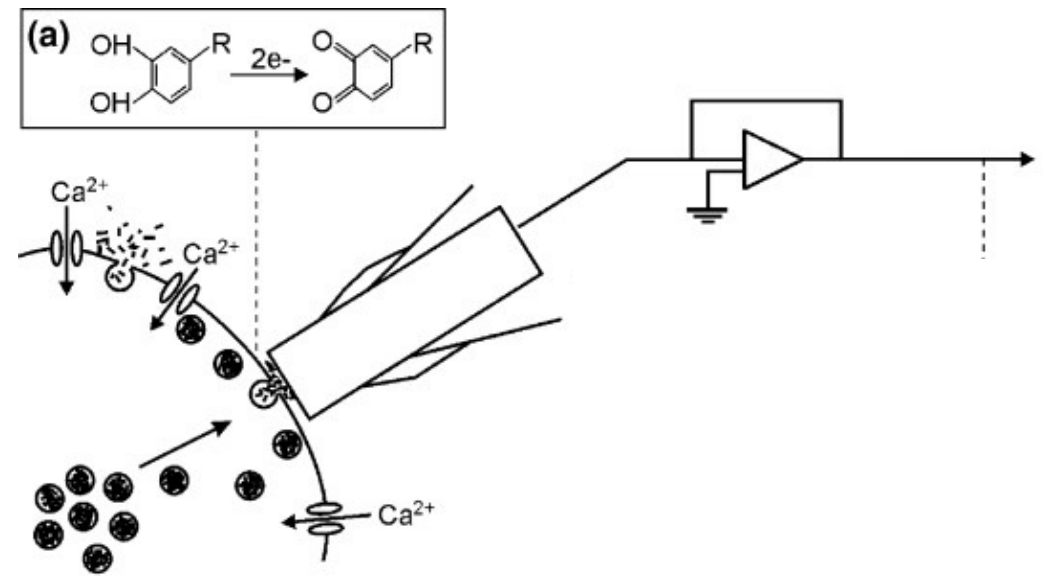

Fig. 1.2. Experimental set-up of carbon fiber amperometry

(a) oxidation reaction of a typical catecholamine (Westerink et al., 2006). Reproduced with copyright permission from John Wiley and sons.

dopamine) are readily oxidized by electrochemical electrodes. Detection of catecholamines from single cells using electrochemical electrodes was pioneered in the Wightman lab (Chow et al., 1992a; Finnegan and Wightman, 1995;

Wightman et al., 1991). In this approach a microelectrode is placed immediately adjacent to a single cell and held at a potential sufficient to oxidize catecholamines released from a single vesicle while recording the faradaic 
current (amperometry). Typically, two electrons are transferred for each catecholamine molecule that is oxidized on the electrode surface, therefore quantification of the faradaic current allows the time course and amount of catecholamine released from individual vesicles to be measured (Finnegan and Wightman, 1995; Travis and Wightman, 1998; Wightman et al., 1991). The earliest amperometric experiments were performed using carbon fiber electrodes (Chow et al., 1992a; Leszczyszyn et al.) pressed firmly on the surface of a cell that was stimulated to release neurotransmitter. Fig. 1.2. shows the experimental setup of single-cell amperometry using a carbon fiber electrode. Calcium signaling triggers exocytosis, the carbon fiber electrode is pressed against the cell membrane and records transmitter released from a single vesicle. Fig. 1.2. (a) shows the oxidation reaction of a catecholamine molecule. Owing to the high signal-to-noise ratio and high temporal resolution of amperometry, it is possible to resolve a very small number (as low as 600) of neurotransmitter molecules released (Wightman, 2015) with millisecond time resolution.

\subsubsection{Temporal dynamics of a single-cell exocytotic event}

In a typical amperometric recording, the electrode current assumes a baseline value until transmitter oxidizes on the electrode, following which, a rise in amperometric current can be observed as a "spike". Three distinct stages (depicted in Fig. 1.3.) have been reported within an exocytotic event recorded electrochemically from a single-vesicle, using carbon fiber electrodes (Finnegan and Wightman, 1995; Schroeder et al., 1996), (a) a small increase in current amplitude, corresponding to flux of transmitter via the fusion pore (pre-spike foot 
signal), (b) a rapid rise to a peak amplitude, corresponding to flux increase through an expanded fusion pore; (c) an exponentially decaying phase, attributed to depletion of transmitter from the vesicle. The rate of decay also depends on the rate of dissociation of catecholamine from the tightly packed intra-vesicular matrix or gel (Schroeder et al., 1996).

Common parameters measured from an amperometric spike include the time to

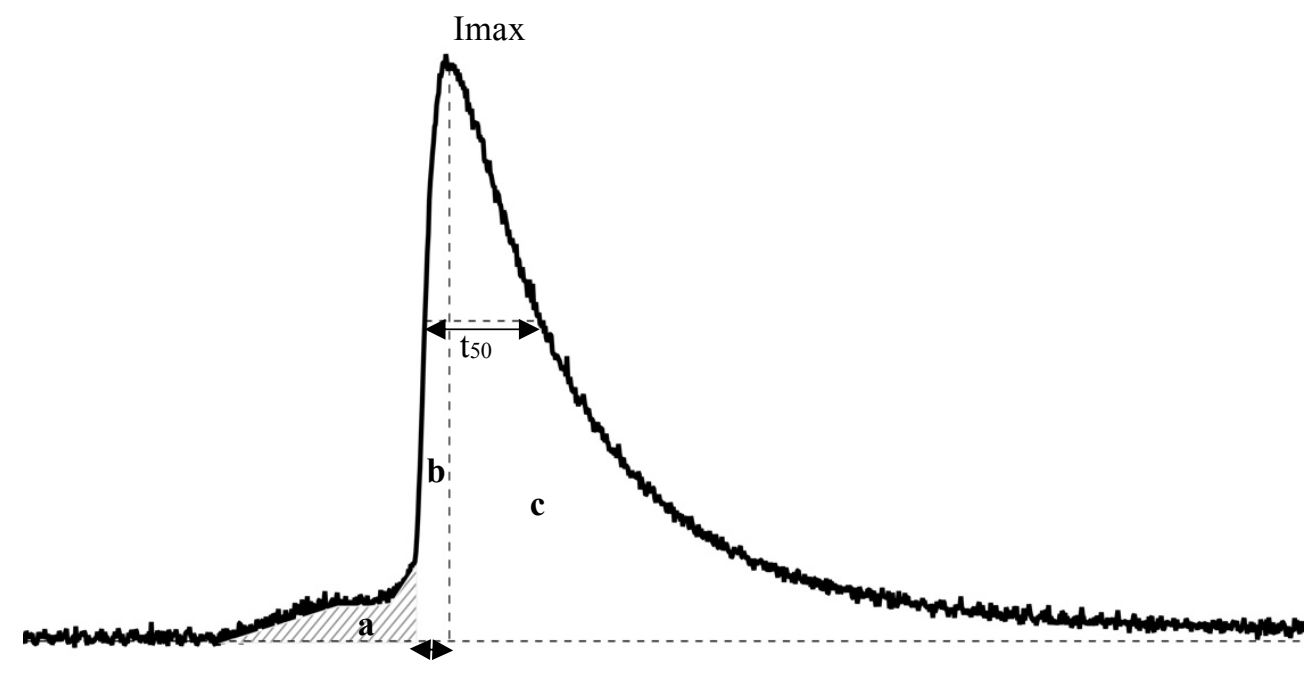

Fig. 1.3. Three distinct phases in an exocytotic event

(a) small rise in current(foot-signal) (b) rapid rise in current to a peak Imax (c) exponential decay of current. Full-width-half-maximum( $\left(t_{50}\right)$ is shown. The time to peak $t_{\text {peak }}$ is the duration of the rising phase (b).

peak $\left(t_{\text {peak }}\right)$ and full-width-half-maximum $\left(t_{50}\right)$. These temporal parameters signify the duration of the fusion pore expansion $\left(t_{\text {peak }}\right)$ and the duration of spike amplitude exceeding $50 \%$ of its maximum amplitude $\left(t_{50}\right)$. Other important parameters of interest are, charge (Q) which relates to amount of cargo released and can be computed as the area under the amperometric spike, and $\mathrm{I}_{\max }$ or peak amplitude, which is the maximal flux through the fusion pore. Qfoot, cargo 
released via the narrow fusion pore and $t_{\text {foot}}$, the duration of the narrow fusion pore can also be inferred from the foot-signal in the amperometric recording.

\subsubsection{Micro-electrode arrays}

\subsubsection{Limitations of Carbon Fiber amperometry}

Carbon was the first choice of electrode material to measure catecholamine release owing to its excellent electrochemical properties (Chow et al., 1992a; Wightman et al., 1991) (Finnegan and Wightman, 1995). In this method, carbon fibers are pressed onto the plasma membrane and held at a positive potential sufficient to oxidize released catecholamine molecules. CFEs have limitations in their capabilities for high-throughput measurements because CFEs can only be used to record amperometric currents from one cell at a time. Moreover, a micromanipulator is required in order to co-localize the electrode tip and the plasma membrane, requiring fine manipulation and considerable time consumption. Cellcell variability in quantal exocytosis necessitates repetition of CFE experiments for a large number of cells (Colliver et al., 2000), making it cumbersome and slow. Another disadvantage is that, CFEs only record from a fraction of the cell surface (12-15\%) (Chen et al., 2003) whereas exocytosis occurs over a significant fraction of the cell surface for chromaffin cells. Additionally, it is not possible to combine Total Internal Reflection (TIRF) microscopic imaging of individual vesicles with carbon fiber amperometric recording from the imaged vesicles since the electrodes are opaque and interfere with the imaging. 


\subsubsection{Advantages of Micro-electrode arrays}

Micro-electrode arrays provide several advantages over traditional CFE amperometry. Recordings can be made from several cells at the same time in a convenient manner hence enabling high-throughput measurement.

In order to account for cell-cell variability, each electrode should record from a single cell. To this effect, micro-fluidic cell chambers that trap single cells can be integrated onto micro-electrode arrays. For example, pico-liter sized wells to trap cells over working electrodes were implemented by the Gillis group (Liu et al., 2011). Another example, to automate the targeting of single cells to working electrodes, is a micro-fluidic trap designed and implemented by Gillis and Gangopadhyay groups using micro-systems fabrication techniques (Gao et al., 2009). In this device (Gao et al., 2009), cell solution was loaded through an inlet reservoir and allowed to exit through a parallel exit reservoir only after trapping cells in a narrow passage, orthogonal to both reservoirs, consisting of active docking sites where quantal release was measured on platinum micro-electrodes. In a typical amperometric spike, the maximal flux $\left(\mathrm{I}_{\max }\right)$ decreases with distance of the electrode from the cell. This is attributed to diffusional delay of transmitter reaching the electrode, and hence necessitates a reduction in electrode-cell distance. In traditional CFE amperometry, the electrode is pressed onto the cell membrane. In MEAs, the cell is localized to the electrodes and the cell-electrode adhesion can be further improved using surface-modification techniques. For example, the Gillis (Barizuddin et al., 2010) and Gangopadhyay (Liu et al., 2011) groups employed cytophilic materials to promote cell-adhesion to electrodes. 
Other advantages of MEAs are the fabrication of transparent electrodes to facilitate simultaneous Total Internal Reflection fluorescence(TIRF) microscopic imaging of individual vesicles tagged with fluorophores and multi-site detection on a single-cell for spatial localization of vesicle release sites.

\subsubsection{Micro-electrode materials}

One of the powerful features of micro-electrode arrays is the large choice of electrode-materials. Table 1 summarizes a comparison of various electrode materials including gold (Au), Indium-Tin-Oxide (ITO), Boron-doped-diamond (BDD) and Nitrogen doped Diamond-like-Carbon (DLC: N) (Gillis et al., 2017).

Desirable properties of electrode materials include, polarizability, low background current, high sensitivity, transmittance and ease of fabrication (Gillis et al., 2017). Polarizability, or the ability of an electrode material to redistribute charges when placed in an electrolyte solution, in such a manner as to create a strong electric field at the interface of the electrode and the solution, is important for amperometry. A lower background current that signifies better polarizability is observed in ITO, BDD (Kiran et al., 2012) and CFEs (Chow et al., 1992a) than in Au. Sensitivity, measured by the number of electrons transferred per catecholamine molecule is lower in ITO than the $\mathrm{Au}, \mathrm{BDD}$, and CFE electrodes (Kisler et al., 2012). CFEs have several good electrochemical features including sensitivity and low background current but carbon is not compatible with multi- 
Table 1.1. Comparison of four electrode materials for micro-electrode fabrication

\begin{tabular}{|c|c|c|c|c|c|}
\hline Property & Au & ITO & BDD & DLC:N & CFE \\
\hline $\begin{array}{l}\text { Background } \\
\text { current }\end{array}$ & $\begin{array}{l}\sim 0.1-0.3 \\
\mathrm{pA} / \mu \mathrm{m} 2\end{array}$ & $\begin{array}{l}\sim 0.01-0.03 \\
\mathrm{pA} / \mu \mathrm{m} 2\end{array}$ & $\begin{array}{l}\sim 0.01- \\
0.03 \\
\text { pA/ } / \mu \mathrm{m} 2 \\
\text { (Kiran et } \\
\text { al., } 2012 \text { ) } \\
\end{array}$ & $\begin{array}{l}\sim 0.02- \\
0.16 \\
\text { pA/ } / \mu \mathrm{m} 2 \\
\text { (Gao et al., } \\
2008)\end{array}$ & \begin{tabular}{|l|}
$\sim 0.01-$ \\
0.03 \\
$\mathrm{pA} / \mu \mathrm{m} 2$ \\
(Chow et \\
al., $1992 \mathrm{a})$ \\
\end{tabular} \\
\hline $\begin{array}{l}\text { Sensitivity } \\
\text { (number of } \\
\text { electrons } \\
\text { transferred per } \\
\text { catecholamine } \\
\text { molecule) } \\
\text { (Kisler et al., } \\
\text { 2012) }\end{array}$ & $2.0-2.7$ & $\sim 1.5$ & 1 & $2.0-2.7$ & $2.0-2.7$ \\
\hline $\begin{array}{l}\text { Transmittance } \\
\text { (Kisler et al., } \\
\text { 2012) }\end{array}$ & $\begin{array}{l}50-70 \% \text { was } \\
\text { at } 400 \mathrm{~nm} \text { for } \\
\text { a } 13-\mathrm{nm}- \\
\text { thick } \mathrm{Au} \text { film }\end{array}$ & $\begin{array}{l}\sim 80 \% \text { at } 360 \\
\mathrm{~nm} \text { and } \\
>90 \% \text { for } \\
\text { wavelengths } \\
\text { above } 400 \\
\text { nm for a } 100- \\
\text { nm-thick } \\
\text { ITO film }\end{array}$ & $\begin{array}{l}\sim 50 \% \text { at } \\
350 \mathrm{~nm} \text { for } \\
\text { a } 200 \mathrm{~nm} \\
\text { thick film }\end{array}$ & $\begin{array}{l}\sim 70 \% \text { at } \\
400 \mathrm{~nm} \text { for } \\
\text { a } 40 \mathrm{~nm} \\
\text { thick DLC } \\
\text { on top of a } \\
100 \mathrm{~nm} \\
\text { ITO film }\end{array}$ & $\begin{array}{l}\text { Not } \\
\text { transparent }\end{array}$ \\
\hline $\begin{array}{l}\text { Ease of micro- } \\
\text { electrode array } \\
\text { fabrication }\end{array}$ & $\begin{array}{l}\text { Straightforw } \\
\text { ard through } \\
\text { photolithogra } \\
\text { phy }\end{array}$ & $\begin{array}{l}\text { Straightforw } \\
\text { ard through } \\
\text { photolithogra } \\
\text { phy }\end{array}$ & $\begin{array}{l}\text { Specialize } \\
\mathrm{d} \\
\text { equipment } \\
\text { to deposit } \\
\text { via hot } \\
\text { filament or } \\
\text { microwave } \\
\text { plasma } \\
\text { chemical } \\
\text { vapor } \\
\text { deposition } \\
\text { (Macphers } \\
\text { on, 2015) }\end{array}$ & $\begin{array}{l}\text { Care } \\
\text { required to } \\
\text { avoid } \\
\text { removal of } \\
\text { DLC layer } \\
\text { by plasma } \\
\text { etching } \\
\text { (Gao et al., } \\
2008 \text { ) }\end{array}$ & $\begin{array}{l}\text { Typically, } \\
\text { incompati } \\
\text { ble with } \\
\text { batch } \\
\text { processing } \\
\text {. }\end{array}$ \\
\hline
\end{tabular}

array batch processing techniques even when attempted with forms like carbon paste (Martin et al., 2001) (Rossier Joël et al., 1999) or screen printed carbon (Wang and Pumera, 2002) (Wang et al., 2001b). Moreover, carbon electrodes are 
not transparent. Indium tin oxide (ITO) and nitrogen doped diamond like carbon (DLC:N) deposited on ITO electrodes exhibit excellent transmittance (Kisler et al., 2012) and are hence suitable for combination with fluorescence imaging experiments (Amatore et al., 2006. Gold electrodes can also be transparent when fabricated as thin layers. However, such thin fabrication would compromise the robustness of the electrode. Another advantage of MEAs is the capability to surround cells with four or more electrodes(Andrew et al., 2002), in order to spatially localize the release site. This technique, when combined with fluorescence imaging experiments (Amatore et al., 2006; Zhao et al., 2013), can enable exact correlation of an amperometric event with a single vesicle release.

\subsubsection{Different modes of fusion resolved in amperometry}

Different modes of fusion (van Kempen et al., 2011) have been reported in bovine, pc-12 and mice chromaffin cells through amperometry, patch-clamp and fluorescence experiments, namely (a) full fusion (Albillos et al., 1997; de Toledo et al., 1993a; Perrais et al., 2004; Taraska et al., 2003) - where the vesicle membrane fuses with the cell membrane and releases the entire cargo observable as a typical tall spike in amperometry (b) kiss-and-run exocytosis (1985;

Ceccarelli et al., 1973; Fesce et al., 1994; Klyachko and Jackson, 2002) - a type of partial fusion involving brief fusion followed by abrupt endocytosis, observable as small and fast flickers in amperometric currents. (c) kiss-and-stay exocytosis - In this mode, the vesicle fuses and stays open at a narrow diameter, slowly releasing its contents until it decays without further expansion. The observation of almost rectangular shaped, small amplitude long duration "stand- 
alone foot" signals is attributed to kiss-and-stay exocytosis. Fig. 1.4. Illustrates the three major modes of exocytosis and the corresponding signals. Some cells exhibit a phenomenon known as compound exocytosis (1990; Ichikawa, 1965; Pickett and Edwardson, 2006; Scepek and Lindau, 1993) wherein vesicles fuse with each other to release a larger cargo to the extracellular space. These events have been reported as large amplitude amperometric spikes (Bokvist et al., 2000)

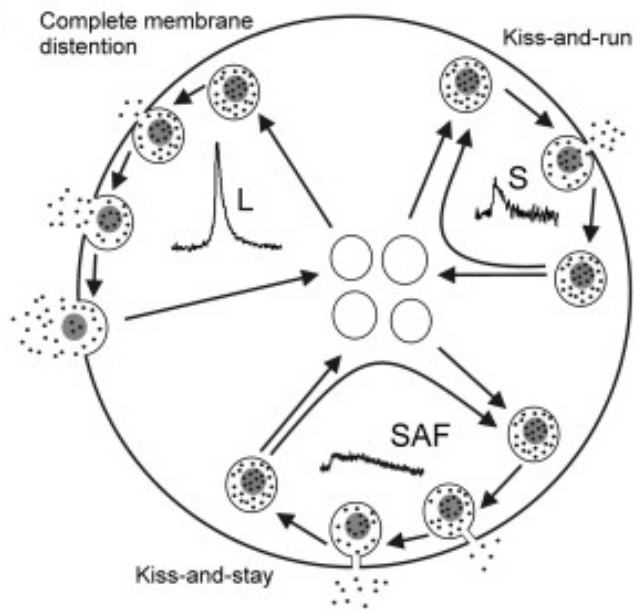

Fig. 1.4. Three distinct modes of fusion, full-fusion, kiss-and-run and kissand-stay

Reproduced from (van Kempen et al., 2011) with copyright permission from Elseiver

in mouse pancreatic $\beta$ cells.

\subsubsection{Stages of fusion pore resolved in amperometry}

A typical amperometric recording consists of a wavering baseline current interspersed with "spikes" of current corresponding to "bursts" or quanta of neurotransmitter released. Exocytosis occurs in steps such as (a) docking (b) priming and (c) fusion. During docking and priming only a baseline current is observed in amperometry. When fusion of vesicle and cell membranes occurs, two distinct stages are observed (i) fusion pore formation- when a nano-metric 
channel connecting the vesicular contents to the extracellular region, is formed. When the fusion pore begins to form, a small current increase is observed, which can plateau for the duration the pore diameter stays narrow. (ii) fusion pore expansion - when the fusion pore expands, to release the cargo quickly. During fusion and release of neurotransmitter, a typical spike consisting of a fast

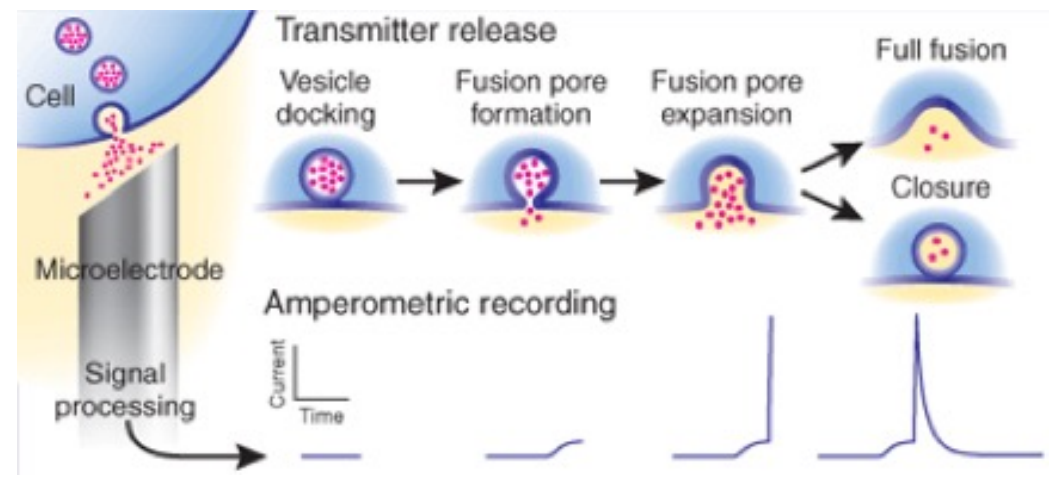

Fig. 1.5. Different stages of fusion and corresponding amperometric trace (from left to right)

The corresponding amperometric currents are shown below. Image reproduced from (Evanko, 2005) with copyright permission from Nature Methods

exponential rise in the current followed by a slower exponential decay. In fast rising, tall spikes, the exponential decay sometimes follows a double exponential course (Wang et al., 2001a). Fig. 1.5. Shows the amperometric traces corresponding to different stages of fusion.

Bovine chromaffin cells have an abundance of secretory vesicles (Winkler et al., 2006) and were hence chosen for amperometry experiments in this study. A secretagogue such as high concentration of $\mathrm{K}^{+}$solution (Yang et al., 2007) was perfused into the cells to depolarize cells and induce exocytosis and gold microelectrode arrays were used to measure the amperometric current. 


\subsection{Automated analysis of amperometric signals $\uparrow$}

\subsubsection{Need for automated analysis}

Spike frequency, which corresponds to the rate of exocytosis, is a useful metric that can be determined by analyzing amperometric recordings. Manual analysis of amperometric currents is the gold standard for locating spikes but is time consuming and subjective. Automated detection of exocytotic spikes is hence necessary for improved speed and accuracy. There are four challenges in the automated temporal identification of exocytotic spikes (a) exocytotic spikes exhibit variation in the rise and decay time constants that depend upon the rate of fusion pore expansion and rate of dissociation of neurotransmitter from the intravesicular matrix respectively; (b) the spikes also exhibit diverse amplitudes, which are governed by the flux of the neurotransmitter traversing the fusion pore; (c) the baseline current is not steady, and tends to increase when secretagogues are perfused into the cells; (d) Successive spikes occurring closely in time, known as "overlapping" spikes are ambiguous to resolve.

\subsubsection{Existing approaches}

The most widely used automated spike detection algorithms for this application detect an event when the derivative of the trace exceeds a set threshold (Mosharov and Sulzer, 2005; Segura et al., 2000). This classical approach is quick and computationally efficient. However, there are two main disadvantages in such an approach. Firstly, data smoothing is required both before and 
after computing the derivative in order to prevent spurious detection. Excessive smoothing may lead to spikes being undetected whereas insufficient smoothing can result in detection of noisy transients as spikes (false positives). The onus is on the user to determine optimal filtering parameters. Secondly, the derivative threshold method is biased against slowly rising spikes, which can go undetected in this approach. Moreover, this method only uses information from a brief interval in the spike, the rapidly rising phase, and does not exploit information from the bulk of the spike time course when the amplitude is declining. An alternative approach to detect exocytotic spikes is to subtract the time-varying baseline from the signal and then detect spikes that exceed amplitude thresholds (Friedrich and Ashery, 2010). However, tracking the time-varying baseline with pA precision requires that the baseline be stable over the averaging time window and relies on user input of the noise level. Also, in an amplitude-based algorithm

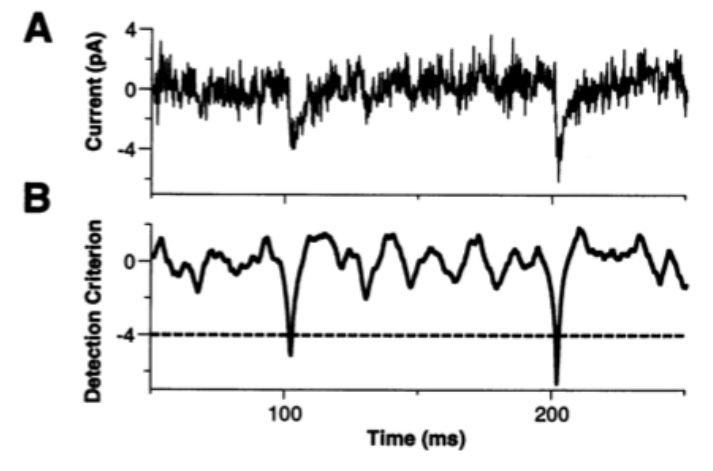

Fig. 1.6. Detection criterion for mini-synaptic events

(A) Two spontaneous mini events (B) corresponding detection criterion. Reproduced from Clement and Bekkers (Clements and Bekkers, 1997) with copyright permission from Elseiver. small-amplitude flickers may be mistaken as spikes (Friedrich and Ashery, 2010), whereas MF approach rejects such flickers because their time courses do not match the templates. Avoiding false positives inevitably makes amplitude-based algorithms more biased against small-amplitude events than template-based 
algorithms.

\subsubsection{Matched filter approach}

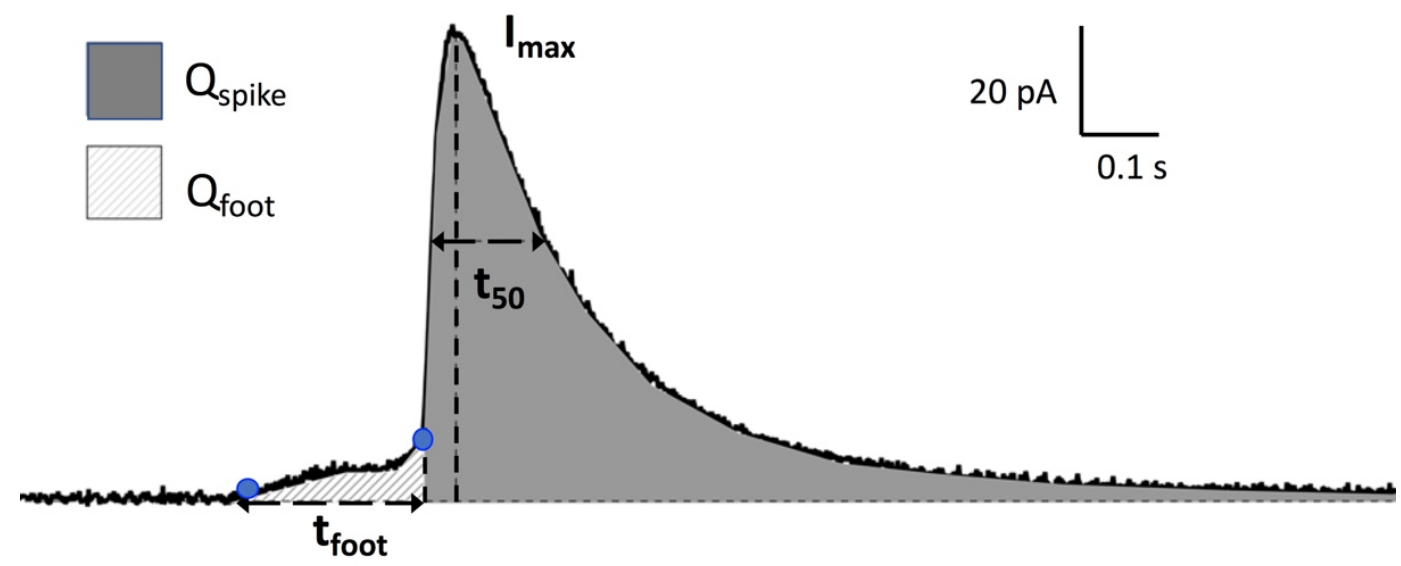

Fig. 1.7. A typical amperometric trace preceded by a pre-spike foot signal

Foot signal (between the blue circles). Spike parameters $\mathrm{t}_{50}, \mathrm{I}_{\max }, \mathrm{Q}_{\text {spike }}$ and foot parameters of $t_{\text {foot }}$ and $\mathrm{Q}_{\text {foot }}$ are shown

Event detection is a common problem in signal processing with a rich literature (Bell, 1993b; Poor, 2013). A powerful approach to detect events in a noisy background is to exploit a priori knowledge about the event time course through use of an idealized event template. Matched filtering refers to the process of correlating the measured signal with the idealized event template in order to detect events when the correlation exceeds a threshold. Whereas matched filtering has been used in detection applications such as radar (Bell, 1993a) and seismic detection (Shearer, 1994) for many years, its use for detecting quantal exocytosis via amperometry has not been systematically studied. Examples of biological signals that have been detected using template-based approaches are extracellular action potentials (Kim and McNames, 2007; Nakamura et al., 2005; Szymanska et al., 2014), electroencephalograph (EEG) signals (Stamoulis and Chang, 2009) 
(Mesbah et al., 2007; Stamoulis and Chang, 2009) and spontaneous post-synaptic electrophysiological events ("minis") (Clements and Bekkers, 1997). In the approach described by Clement and Bekkers, a template is used that represents an ideal postsynaptic mini event. The template is correlated point-by-point throughout the recording and a score is calculated for each time point. Events are detected when the score exceeds a threshold value. Fig. 1.6. Shows a trace containing mini-synaptic events and the corresponding criterion scores. However, detection of amperometric spikes is a more challenging problem than minis because amperometric spikes vary widely in amplitude and time course. This study describes the application of matched-filtering(MF) approach to detect amperometric spikes.

\subsubsection{Estimation of spike parameters}

Another objective of this study was to estimate spike parameters that describe the physiological nature of the release mechanism. The area under the spike $(\mathrm{Q})$ can be used to determine the amount of neurotransmitter released, full width at half maximum $\left(\mathrm{t}_{50}\right)$ can be used to determine flux-duration and the peak current $\left(\mathrm{I}_{\max }\right)$ corresponds to the maximum flux through the fusion pore (Jankowski et al., 1993; Wightman et al., 1991). Fig. 1.7. Shows an amperometric spike with parameters denoted.

\subsubsection{Overlap rejection}

The first step in spike estimation is the rejection of closely successive, overlapping spikes (shown in Fig. 1.8.) which do not decay to baseline and hence their start and end points are ambiguous to estimate. Existing approaches either 
rely on the user to determine the extent of overlap tolerable (Mosharov and Sulzer, 2005) or use arbitrary thresholds on the ratio between amplitudes of adjacent spikes (Friedrich and Ashery, 2010) to determine if a spike is wellseparated or overlapping. We demonstrate a user-independent algorithm to analyze pairs of adjacent spikes and accept or reject them based on a twocondition approach. The first condition rejects the second spike if it occurs prior to the decay of the first spike to a minimum amplitude.

The second condition rejects the first spike if it did not decay to 3 decay time constants. MF template parameters provide accurate seed values for the decay time constants, to help reject overlapping spikes.

\subsubsection{Multi-step curve-fitting}
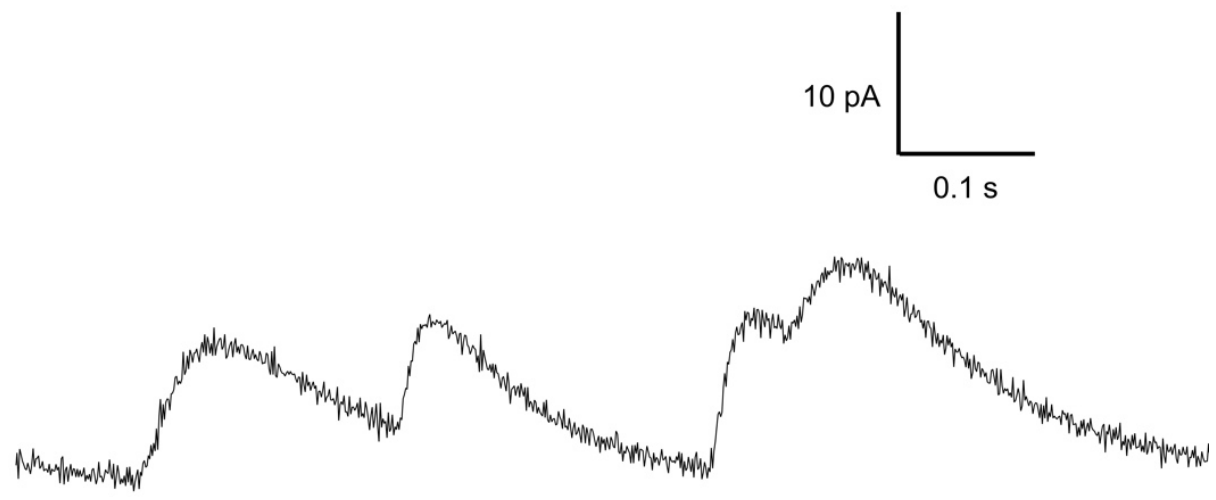

Fig. 1.8. Overlapping spikes

The next step in spike estimation is curve-fitting of spikes so as to obtain "goodfits" to spikes that can be used to calculate spike parameters. Linear least squares curve fitting performed in the MF approach provided excellent spike detection, but for accurate spike estimation, finely tuned non-linear curve fits are required. 
Iterative non-linear curve fitting is highly sensitive to the initial guesses of parameters. Existing spike estimation algorithms rely on line-fitting to an arbitrary segment of the rising phase and auto-seeded non-linear curve fitting of the decaying phase of the spike. We demonstrate that MF template parameters provide good initial seed values for non-linear curve fitting of spikes to an exponential rise followed by multi-exponential decay spike function. Moreover, in order ascertain a high accuracy of curve-fits, we first performed MF-parameterseeded exponential rise fits to the rising phase of the spike and MF-seeded

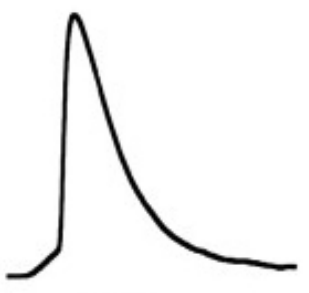

With a "ramp" foot

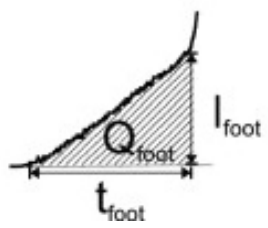

Fig. 1.9. Two types of feet

(Amatore et al., 2009) show, ramp and a ramp + plateau. Reproduced with copyright permission from Elseiver. double/single exponential decay fits to the decaying phase of the spike. The parameters obtained from these two fits were then fed into an overall spike function to obtain "good fits" to spikes. Using spike fit parameters like $\tau_{\mathrm{r}}$ (rise time constant), $\tau_{\mathrm{d}}$ (decay time constant), $\mathrm{a}_{1,2}$ (amplitudes of two decays), $\mathrm{t}_{0}$ (spike offset time) and $\mathrm{b}$ (baseline) spike parameters including $\mathrm{Q}, \mathrm{t}_{50}$ and $\mathrm{I}_{\max }$ were calculated. Histogram of spike parameters were compared against those computed using existing and manual approaches to determine the performance of the algorithm. 


\subsubsection{Pre-spike foot signal analysis}

Some exocytotic events exhibit a small pre-spike feature (Amatore et al.; Amatore et al., 2007; Chang et al., 2017; Chow et al., 1992a; de Toledo et al., 1993a) termed as "foot signal". It appears as a small increase in the current which could then take on the shape of a ramp, a plateau or a ramp followed by a plateau (Amatore et al., 2009).

Through patch-amperometry (Albillos et al., 1997) (combining patch-clamp with amperometry) experiments Chow et al. correlated the occurrence of foot signals to a slow leak of molecules through the narrow fusion pore in the early stages of exocytosis (Chow et al., 1992a).

\subsubsection{Baseline detection}

In order to analyze pre-spike foot signals, the pre-spike baseline current should first be established. Existing approaches (Mosharov and Sulzer, 2005) determine the baseline by performing a steady state search on a filtered amperometric trace and then apply an amplitude threshold of 2.5 times standard deviation of background noise to detect foot beginning points, where the background noise is determined using a "spike-free" segment of the data, indicated by the user. We demonstrate an algorithm that uses two pieces of information obtained from MF spike detection namely (a) spike start time and (b) criterion score to analyze prespike activity. The MF detection approach provided accurate spike start times, which was useful for location and analysis of the pre-spike segment. For spike detection, the MF approach provided a criterion score to describe the extent of 
template-data match. However, this score reaches zero when the least squares amplitude is zero. Hence, the criterion score zero can be used to determine the region of baseline in every pre-spike segment. Once baseline was determined, an amplitude threshold was then applied to detect the beginning of pre-spike foot

signal. The point $\mathrm{t}=\mathrm{t}_{0}$ of the exponentially rising spike was identified to be the end of the pre-spike foot signal. The area under the foot and lifetime of the foot are measures of charge and lifetime of narrow fusion pore and were determined using the above detected foot signal. Mean and median foot-signal parameters were compared with those computed by existing and manual approaches to measure performance.

\subsection{Dissertation objectives and overview}

The three objectives of this study are (a) automated detection of amperometric spikes (b) estimation of spike parameters (c) detection and estimation of foot signals.

This dissertation is composed of five chapters

Chapter 1 provides an introduction to exocytosis mechanisms, electrochemical methods of detection, existing algorithms that analyze amperometric signals and an overview of the new approaches developed in order to study amperometric signals.

Chapter 2 describes the materials and methods used for this study, including bovine chromaffin cell preparation, microelectrode array fabrication, amperometry, matched filter, least squares fitting, receiver operating 
characteristics, overlap rejection, multi-step non-linear spike fitting, baseline detection and pre-spike foot analysis.

Chapter 3 consists of the results of matched filtering for spike detection. ROCs comparing the MF approach to existing algorithms such as derivative threshold and other commercial applications are presented. The MF approach outperforms existing approaches by detecting $40 \%$ more events than traditional approaches (Balaji Ramachandran and Gillis, 2018) and $>95 \%$ of the events for $<5 \%$ false positive rate.

Chapter 4 describes the approaches undertaken to estimate spike parameters such as $\mathrm{t}_{50}, \mathrm{I}_{\max }$ and $\mathrm{Q}$. Histograms of manual and algorithmic estimates are compared. The analysis and estimation of foot signals, foot parameters such as lifetime( tfoot$)$ and charge $\left(\mathrm{Q}_{\text {foot }}\right)$ are presented.

Chapter 5 discusses the outcomes of the MF Detection and Estimation algorithms. Future directions include machine learning approaches to automatically design a template library, analysis of stand-alone foot signals and post-spike foot signals.

†Partially reproduced from Balaji Ramachandran et al(Balaji Ramachandran and Gillis, 2018) 


\section{Chapter 2}

\section{MATERIALS AND METHODS}

\subsection{Fabrication of micro-electrode arrays}

As discussed in Chapter 1 (Section 1.2.1), single-cell amperometry is a powerful technique for measuring quantal exocytosis. In this study, amperometric measurements were carried out using gold multi-electrode arrays. The following sections describe the fabrication and testing of gold micro-electrode arrays.

\subsubsection{Electrode array Fabrication steps}

Gold, a polarizable electrode material with fast electron-transfer kinetics (Zachek et al., 2008) and was used for electrode fabrication. The following were the steps for fabricating a device consisting of 16 electrodes (Liu et al., 2011).

\subsubsection{Sputter coating}

Microscope slides (25 X 75 X 1) mm, (Fisher brand, Fisher Scientific, Pittsburgh, PA, USA (Martin et al., 2001)) were cleaned in four steps a) soaked in acetone for

5 min b) washed with methanol c) washed with isopropanol d) washed with DI water. The cleaned slides were air dried. A 2nm Ti layer was first sputter deposited on the slides following which $\sim 30 \mathrm{~nm}$ thick gold film was sputter deposited. The Ti layer acts as an adhesion film for the Au layer.

\subsubsection{Gold Patterning}

The Au film was patterned using photo-etching processes with S1813 photoresist (Rohm and Haas electronic materials, Philadelphia, PA, USA (Rohm)) as a masking layer. First, the conductor-coated slides were cleaned by sonication in acetone for $10 \mathrm{~min}$ followed by exposure to air plasma (PDC-32G, Harrick 


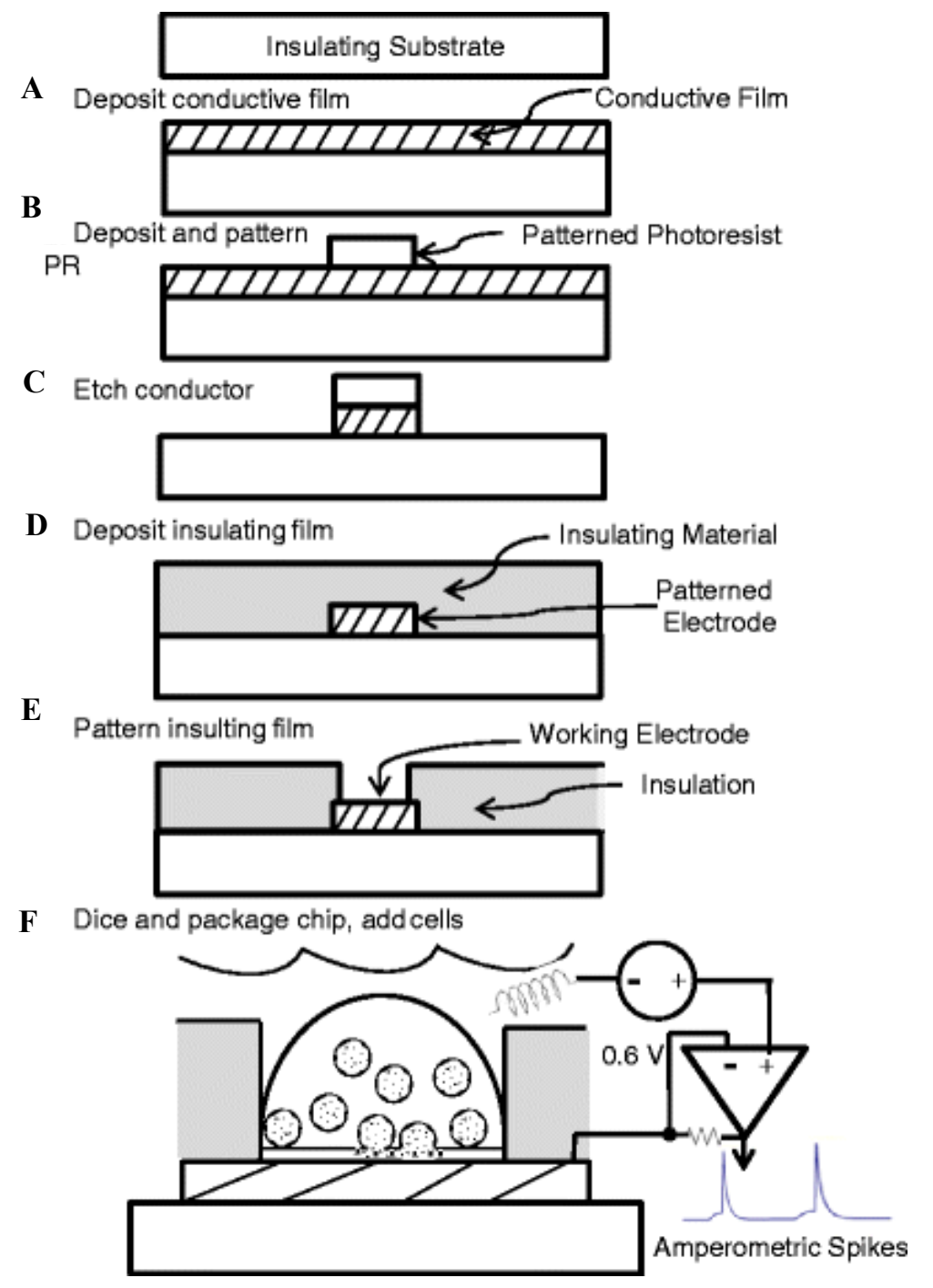

Fig. 2.1. Microelectrode array fabrication steps

A) Gold film sputter deposited on glass substrate. S1813 layer spin coated on gold film and exposed to UV through photomask 1 (B) Patterned photo-resist layer C) Etching of the gold pattern D) SU8 layer spin coated on the sample. UV exposure through Photomask 2 E) A well for trapping the cell is patterned and developed. Reproduced from (Gillis et al., 2017) with copyright permission from Springer Berlin Heidelberg
Scientific Corp.,

Pleasantville, NY,

USA (HarrickSci))

for $1 \mathrm{~min}$ at medium

RF power level.

S1813 photoresist

was then spin coated

(Laurell

Technologies Corp.,

North Wales, PA,

USA (Laurell)) onto

the coated slide at

$2500 \mathrm{rpm}$ for $60 \mathrm{~s}$ to

give a thickness of

$\sim 2 \mathrm{~mm}$. The coated

glass slide was then

baked on a hot plate

at $115^{\circ} \mathrm{C}$ for $2 \mathrm{~min}$.

Then it was exposed

to UV light through

a high resolution (20 000 dpi) transparency mask (CAD/Art services, Inc.

Bandon, OR (CAD)) for 2.6s (1000 W metal halide lamp) and then developed in M351 solution(Rohm and Haas electronic materials (Rohm)) for $\sim 1$ min. Since 
S1813 is a negative photoresist, the development step resulted in removal of this layer from regions exposed to the UV light.

\subsubsection{Insulation of gold film}

At the end of the first UV exposure described above, S1813 layer covering the 16electrode pattern $(50 \mu \mathrm{m}$ widths $)$ and corresponding $2 \mathrm{X} 2 \mathrm{~mm}^{2}$ bonding pads was retained. $\mathrm{Au} / \mathrm{Ti}$ films were wet etched using an Au etching reagent (Aqua regia HNO3:Hcl, 1:4) purchased from Sigma-Aldrich for $\sim 5 \mathrm{~s}$. The etched sample was then shaken in PRS3000 (PRS 3000 from Avantor performance materials (Avantor)) stripping solution to strip away the S1813 layer covering the metalfilm pattern. SU8 2025 photoresist spin coated onto the device at $4000 \mathrm{rpm}$ for 1 min to give a thickness of $\sim 16 \mathrm{~mm}$. Then it was baked on a hot plate at $65^{\circ} \mathrm{C}$ for 3 $\min$ and then at $95^{\circ} \mathrm{C}$ for $5 \mathrm{~min}$.

\subsubsection{Opening of working electrodes}

A second mask was used to create $20 \mu \mathrm{m}$ diameter openings in the SU8 insulation that define working electrodes / cell trapping sites and allow electrical connections to bonding pads arranged around the circumference of the chip. The exposed sample was baked for 3 minutes at $75^{\circ} \mathrm{C}$ and 5 minutes at $95{ }^{\circ} \mathrm{C}$ and then left to cool for 30 minutes. The cooled sample was developed for 6-8 minutes using a small quantity of SU8 developer (MicroChem Corp, Newton, MA, USA (Microchem)) in slow perturbation mode in order to remove residual SU8 covering the wells. Since SU8 is a positive photoresist, areas unexposed to light were etched in the developing solution. The developed sample was then baked at $200{ }^{\circ} \mathrm{C}$ for 10 minutes in order to harden the film and seal cracks on the SU8 
layer. The sample was treated by exposure to air plasma (PDC-32G, Harrick Scientific Corp., Pleasantville, NY, USA (HarrickSci)) for 5 mins at medium RF power level. This step enabled the removal of any residual organic matter or SU8 layer residing on the electrodes.

\subsubsection{Packaging steps}

The glass chips containing electrode arrays need to be packaged to facilitate electrical connection to amplifiers and containment of the cell-containing solution placed on top of the array. A small printed circuit board was used to facilitate electrical connection of the electrodes to the potentiostats. Electrical connections between the bonding pads on the circumference of the electrode array chip and connection pads on the PCB were made with conductive silver epoxy. Since the working surface of the electrode array chip faces the PCB, a $7 \mathrm{~mm}$ diameter hole was fabricated on the PCB to allow access of cells and solution to the array in a "flip-chip" configuration. A 3D printed solution chamber was bonded to the glass chip using a cyanoacrylate adhesive to ensure that the cell bath solution does not contact the PCB nor electrical connections on the chip. Electrical connections between the PCB and the amplifier array "head-stage" were made using a header array soldered to the PCB. The entire assembly was then packaged in a 3D printed cassette enclosure.

\subsubsection{Cyclic voltammetry}

Cyclic voltammetry(CV) (Elgrishi et al., 2018; Mabbott, 1983) is the technique of ramping the holding potential of the electrode between two values in order to cyclically reduce and oxidize the analyte and thereby observe the resulting 
cathodic and anodic currents, respectively Cyclic voltammetry with a well-

behaved redox analyte such as ferricyanide is used to confirm the electrochemical

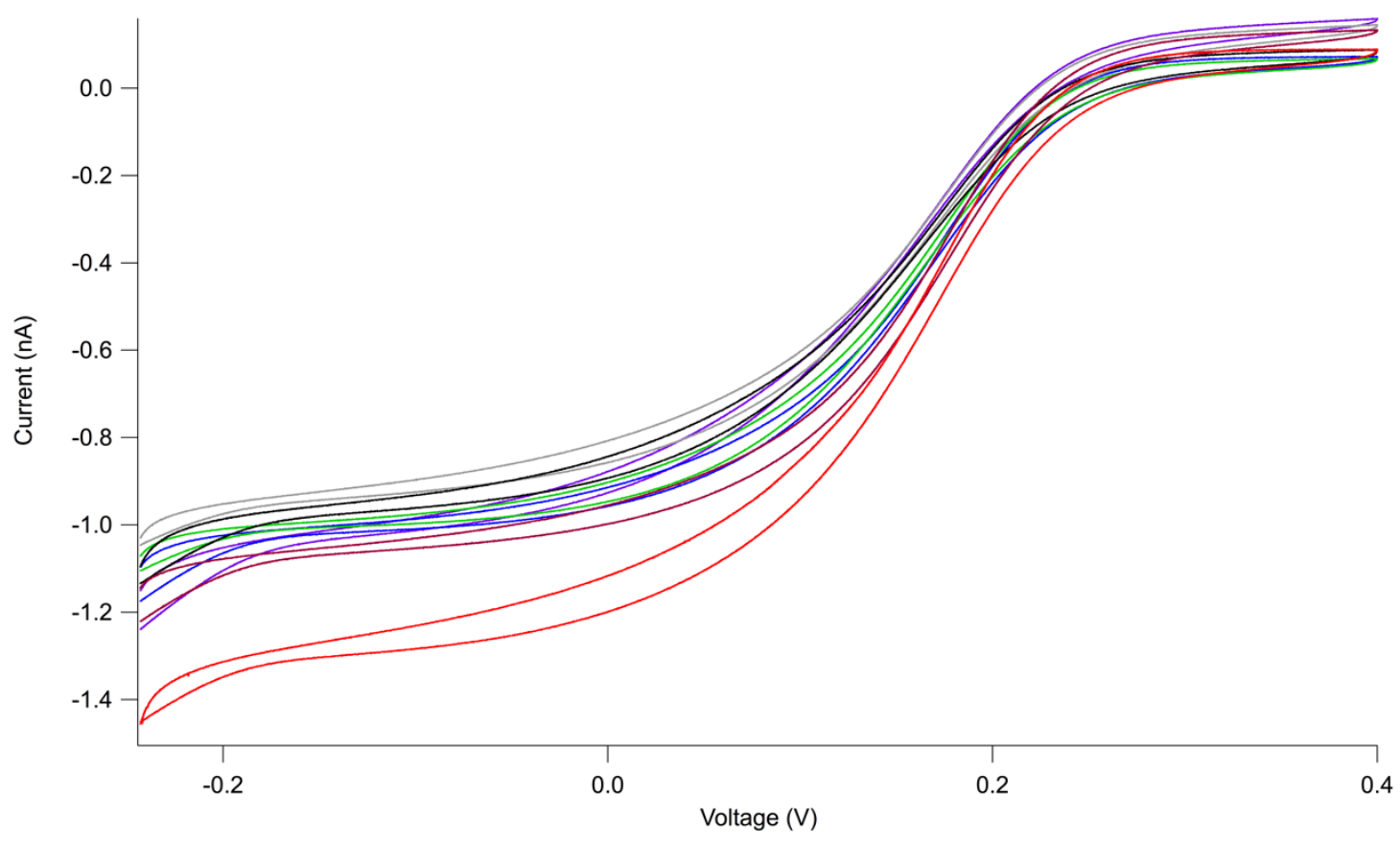

Fig. 2.2. Cyclic voltammograms

Shows the Cyclic voltammograms of a few electrodes using Ferricyanide as the test analyte $\left(1 \mathrm{mM} \mathrm{K}_{3} \mathrm{Fe}(\mathrm{CN})_{6}\right.$ in $0.1 \mathrm{M} \mathrm{KCl}, \mathrm{pH}=3$, scan rate of $\left.10 \mathrm{mV} / \mathrm{s}\right)$, demonstrating steep current changes in response to voltage ramps. Each color corresponds to a $\mathrm{CV}$ curve from an electrode in the device

sensitivity of fabricated electrodes.

For instance, an electrode partially covered with photoresist residue would exhibit a shallow current increase in response to the ramping voltage whereas an electrode with a clean surface exhibits steep current changes. Fig. 11. Shows CV plots for a few electrodes on a gold micro-electrode array device. 


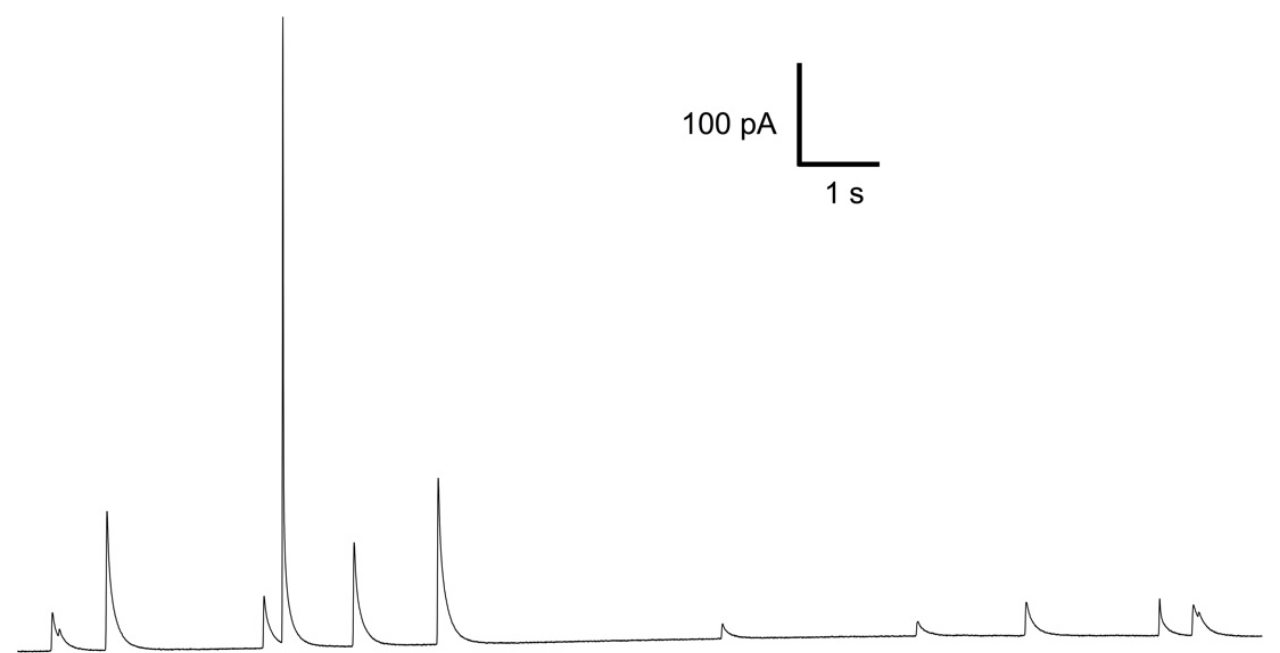

Fig. 2.3. Amperometric trace obtained from an electrode

Amperometric trace obtained from a bovine chromaffin cell using a gold microelectrode array device. Each spike is a result of a single-vesicle release event

\subsection{Experimental recordings}

Chromaffin cells were isolated from bovine adrenal glands and cultured as described previously (Yang et al., 2007). The cell bath solution consisted of (in $\mathrm{mM}): 150 \mathrm{NaCl}, 5 \mathrm{KCl}, 2 \mathrm{CaCl} 2,1.2 \mathrm{MgCl} 2,10 \mathrm{HEPES}$, and 11 glucose, $\mathrm{pH}$ 7.2. A solution with an elevated potassium concentration was used to depolarize cells and induce exocytosis (in $\mathrm{mM}$ ): $55 \mathrm{NaCl}, 100 \mathrm{KCl}, 5 \mathrm{CaCl} 2,2 \mathrm{MgCl} 2,10$ HEPES, and 10 glucose, titrated to $\mathrm{pH} 7.2$ with $\mathrm{KOH}$. Amperometric recordings from bovine chromaffin cells were made with either carbon fiber microelectrodes (ALA Scientific, East Farmingdale, NY, USA (van Kempen et al., 2011)(van Kempen et al., 2011)(van Kempen et al., 2011)(van Kempen et al., 2011)(van Kempen et al., 2011) (Liu et al., 2011) or self-made micro-fabricated Au electrode arrays (Chen et al., 2003; Kisler et al., 2012; Liu et al., 2011). Recordings were filtered at $3 \mathrm{kHz}$ and sampled at $10 \mathrm{k}$ samples $/ \mathrm{s}$. In most recordings, every 10 
points were averaged to result in a "decimated" sampling rate of $1 \mathrm{k}$ samples $/ \mathrm{s}$ and a $-3 \mathrm{~dB}$ bandwidth of $\sim 220 \mathrm{~Hz}$. The decimated sampling rate of $1 \mathrm{kHz}$ is sufficient to resolve fast events in chromaffin cells and results in faster computational speed. Recordings made from mouse chromaffin cells were kindly provided by the laboratory of Kevin Currie (Vanderbilt Univ.) and obtained as previously described (Jewell et al., 2011). Recordings were filtered at $2 \mathrm{kHz}$ and sampled at 10k samples/s.

\subsection{Matched filter detection of amperometric spikes $\uparrow$}

Linear regression is a familiar technique whereby a set of data points is fitted by a line with slope $a$ and intercept $b$ to minimize the sum of squared errors between the data and the fit Fig. 2.4.(A) A straightforward extension of this Least Squares approach is to fit a set of data $y(t)$ consisting of $N$ points with a template function $(f(t))$ to yield an amplitude $a$ and offset $b$ given by Fig. 2.4.(B):

$$
\hat{y}(t)=a f(t)+b(\text { Equation 1) }
$$

where $a$ and $b$ are the least-squares values given by:

$$
\begin{gathered}
a=\frac{\sum f(t) y(t)-\sum f(t) \sum y(t) / N}{\sum f^{2}(t)-\sum f(t) \sum f(t) / N}(\text { Equation 2) } \\
b=\sum y(t)-a \sum f(t) \text { (Equation 3) }
\end{gathered}
$$

The standard error of the least-squares fit is given by:

$$
S E=\sqrt{\frac{\sum(y(t)-\hat{y}(t))^{2}}{N-1}}(\text { Equation 4) }
$$

A criterion score for the fit is calculated as the ratio (Clements and Bekkers, 1997)

$$
\text { criterion score }=\text { a/SE (Equation 5) }
$$


and thus, is a measure of the "signal-to-noise" ratio of the fit, or how strongly the segment of the data is represented by the template. This is repeated by moving the template point-by-point along the data trace in order to produce a criterion score for every possible data segment. A spike is detected each time the criterion score exceeds a threshold, and the time when the criterion score reaches a peak value is the most likely starting time for the spike. It should be noted that even when the least-squares fit appears poor by visual inspection, it can produce a criterion score sufficiently large to detect the spike with high confidence.

\subsubsection{Template waveform +}

Amperometric spikes can often be adequately fit by a function consisting of an exponential rise with time constant $\tau_{r}$ followed by an exponential decline with a much slower time constant $\left(\tau_{d}\right)$ of the form:

$$
\begin{gathered}
f(t)=\left(1-\exp \left(-\frac{t}{\tau_{r}}\right)\right) * \exp \left(-\frac{t}{\tau_{d}}\right)(\text { Equation 6) } \\
; t>0
\end{gathered}
$$

The template contains a brief pedestal before the exponential rise (Clements and Bekkers, 1997), which helps produce a more accurate determination of the baseline b, and, more importantly, helps to ensure that a high criterion score (good fit) is only obtained when the template is precisely aligned in time with the spike found in the data.

A pedestal of duration $t_{p}$ is defined as:

$$
f(t)=0 ;-t_{p}<t<0
$$

\subsubsection{Selection of template waveforms +}


Four templates are sufficient to detect essentially all amperometric spikes in both bovine and mouse chromaffin cells (see Chapter 3). The rising and falling time constants were selected by looking for clustering of time constants found by fitting spikes from representative recordings. Template 1 has a slow rise $\left(\tau_{\mathrm{r}}=50\right.$ $\mathrm{ms}$ for bovine, $70 \mathrm{~ms}$ for mice) and slow decay ( $\tau_{\mathrm{d}}=150 \mathrm{~ms}$ for bovine, $350 \mathrm{~ms}$ for mice). Template 2 has a slow rise ( $\tau_{\mathrm{r}}=30 \mathrm{~ms}$ for bovine, $70 \mathrm{~ms}$ for mice) and an intermediate decay time ( $\tau_{\mathrm{d}}=80 \mathrm{~ms}$ for bovine, $180 \mathrm{~ms}$ for mice). Template 3 has a fast rise $\left(\tau_{\mathrm{r}}=3 \mathrm{~ms}\right.$ for bovine, $10 \mathrm{~ms}$ for mice) and fast decay $\left(\tau_{\mathrm{d}}=8 \mathrm{~ms}\right.$ for bovine, $20 \mathrm{~ms}$ for mice) and template 4 has a fast rise $\left(\tau_{\mathrm{r}}=3 \mathrm{~ms}\right.$ for bovine, $10 \mathrm{~ms}$ for mice) and a slow decay ( $\tau_{\mathrm{d}}=80 \mathrm{~ms}$ for both bovine and mice). All templates are normalized to unit peak amplitude and have $20 \mathrm{~ms}$ pedestals. For bovine chromaffin cells, templates are $200 \mathrm{~ms}$ in duration, whereas mice spike templates are either $250 \mathrm{~ms}$ or $380 \mathrm{~ms}$ in duration. Reducing the template duration to 200 ms did not adversely affect performance (data not shown).

\subsubsection{Comparison with other software $\uparrow$}

Software developed by Eugene Mosharov (Mosharov and Sulzer, 2005) was used as the default derivative-threshold (DT) approach to detecting spikes. This software requires user-specified smoothing frequency, background region and peak threshold. A spike-free region in the amperometric trace was input to be the background region. The spike detection program was run at thresholds ranging from 0-80 pA/ms. ClampFit (Molecular Devices, Sunnyvale, CA, USA) (ClampFit) (ClampFit) (ClampFit) (ClampFit) (ClampFit) a commercial spike detection application, uses templates selected by the user to detect spikes. The 
input templates applied were the same as those used for MF approach. The detection threshold was varied from 0 to 20 and the detection performance was measured at each threshold. MiniAnalysis (Synaptosoft) Fort Lee, NJ, USA, is another commercial spike detection application that uses a derivative threshold approach. This spike detection routine was run at thresholds ranging from 0 to 20 $\mathrm{pA} / \mathrm{ms}$.

\subsubsection{Quantification of detection performance using receiver operating characteristic plots $\uparrow$}

The performance of various spike-detection algorithms was measured using Receiver Operating Characteristic (ROC) plots. The two important indicators of performance are the ability to detect actual spikes (high sensitivity) while minimizing false positives (high specificity). Sensitivity is quantified by the detected fraction, defined as:

Detected Fraction $=($ Num. of true spikes detected $) /($ Total num. of actual spikes in the recording) (Equation 7)

Lack of specificity is quantified by the false-positive fraction, defined as:

\section{False-positive fraction $=($ Num. of false positives detected $) /($ Num. of true spikes detected + Num. of false positives detected) (Equation 8)}

As the threshold value for detection is increased, the false-positive fraction decreases (higher specificity), however, the detected fraction also decreases (lower sensitivity). Therefore, the tradeoff between specificity and sensitivity is 


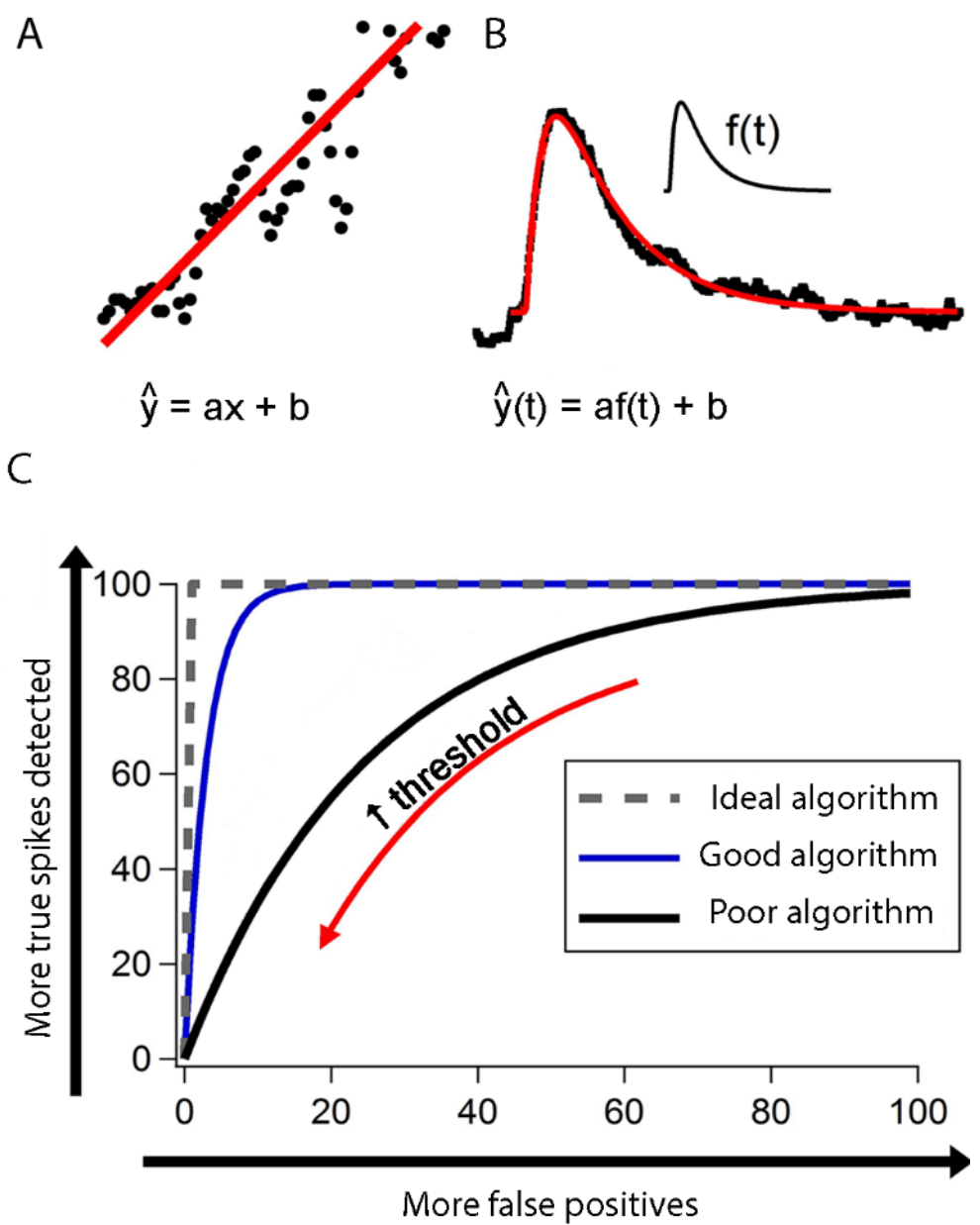

Fig. 2.4. Least squares can fit an amperometric spike with a template function and a receiving operator characteristic plot can quantify the performance of detection algorithms

(A) Least squares fit of a line (red) to sample data (black) to yield the slope $(a)$ and y intercept $(b)$. (B) Least-squares fit of a function $f(t)$ to an amperometric spike to yield the spike amplitude $(a)$ and offset/baseline (b). (C) A receiver operating characteristic is a plot of sensitivity vs specificity as the threshold for detection varies from low to high. A higher threshold tends to reduce false positives at the expense of missing events (lower sensitivity). An ideal detection algorithm (dotted gray) detects $100 \%$ of spikes with $0 \%$ false positives when a proper threshold is used; a poor performing test will resemble the solid black curve because detecting a large fraction of the events with a lower threshold invariably leads to many false positives; a good test will resemble the solid blue curve. 
quantified by plotting the detected fraction versus the false-positive fraction as the threshold value is increased from a low to a high value (Receiver Operating Characteristic, Fig. 2.4(C). An ideal detection algorithm will resemble the dashed curve in Fig. 2.4(C) and detect 100\% of events with $0 \%$ false positives (upper left corner of ROC) when an appropriate threshold is used. A spike detected by an algorithm is considered "true" if the rising edge is within a defined time tolerance of the rising edge inevitably accompany detection of a large fraction of events. In order to quantify the detected fraction and false-positive fraction, one must define the true condition, i.e., identify all the actual spikes recordings as the "gold standard" for identifying spikes. Cases where it is ambiguous whether a signal is a pre-spike foot or in the recording. We used manual analysis of an independent spike were determined manually. The tolerance was set to be the duration of the template. Algorithms were implemented using Igor Pro software (Wavemetrics, Lake Oswego, OR, USA).

\subsubsection{Estimation algorithm}

Fig. 2.5(A) presents a typical amperometric spike recorded from a bovine adrenal chromaffin cell and common parameters to be estimated such as full width half maximum duration (t50), peak amplitude (Imax) and area (Q). Pre-spike foot area (Qfoot) and duration (tfoot) are also indicated.

Fig. 2.5(B) depicts the overall process flow of the parameter-estimation approach. The first step in spike estimation is the identification of a spike. Our previously described matched-filtering algorithm uses four prototype spike templates that vary in time course. Each template is least-squares fit to all possible segments of 
A
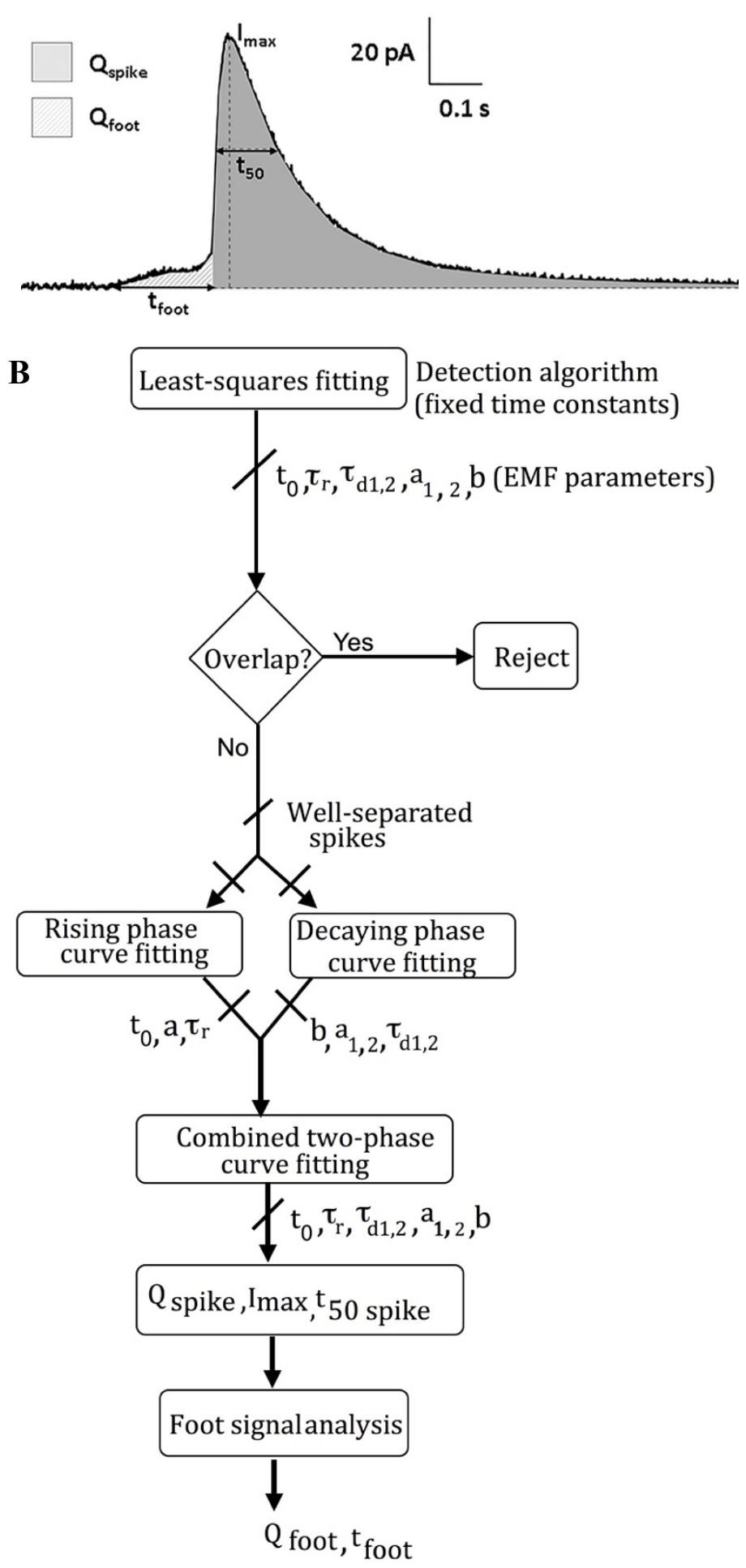

Fig. 2.5. Spike and pre-spike estimation algorithm

(A) An amperometric spike preceded by a pre-spike foot. Spike parameters Q, Imax, $t_{50}$ and foot parameters troot, $Q_{\text {foot }}$ are indicated.

(B) Overall schematic of estimation algorithm. EMF template parameters were seeded into an overlap rejection algorithm. Well-separated spikes were fit with a (i) rising phase function (ii) decaying phase function. Parameters from the rising and decaying phase fits seeded a combined function that fit both phases of spikes. Q, $I_{\max }$ and $t_{50}$ were computed for spikes. Prespike foot signals were estimated to calculate tfoot and Qfoot

data in a recording and the amplitude of the fit of each template is divided by the standard error of the fit to determine a criterion score at each time point. A spike is detected when the criterion score exceeds a threshold, and the template and start time (t0) with the highest criterion scores for the spike are identified. If the sum of two templates produces a

higher criterion score and the smaller template amplitude is at least $10 \%$ the size of the larger template amplitude, the event is classified as "double exponential" 
for parameter identification. Note that fits of templates to the data is by leastsquares, similar to linear regression, and is therefore a closed-form solution without iteration.

The matched-filter detection process yields a set of initial parameter values for the spike including start time (t0), rise time constant ( $\tau r)$, decay time constant(s) $(\tau \mathrm{d} 1,2)$, amplitudes of the exponential decay(s) (a1, a2) and baseline (b). Next, these template parameters are passed onto our overlap rejection algorithm described in detail in section 2.2.1. If adjacent spikes are found to be overlapping, they are rejected for estimation. If adjacent spikes are not overlapping, spike parameters are passed to a two-step curve fitting algorithm. In the first step two iterative curve fits are performed, (i) the rising phase, the interval between the start time and the peak, is fitted by an exponential seeded by detection parameters $\mathrm{t} 0$, $\tau \mathrm{r}$, amplitude (a1+a2) and baseline (b) (ii) the decaying phase of the spike, starting at its peak, is fit by a single or double-exponential decay seeded by determine the baseline and pre-spike foot signals are identified and analyzed to compute tfoot and Qfoot.

\subsubsection{Rejection of overlapping spikes using EMF template parameters}

When a high rate of release is recorded, it is common to have spikes overlapping in time (Wightman et al., 1991). Overlapping spikes are poor candidates for parametric analysis since their time courses and areas cannot readily be resolved. 
$\mathbf{A}$
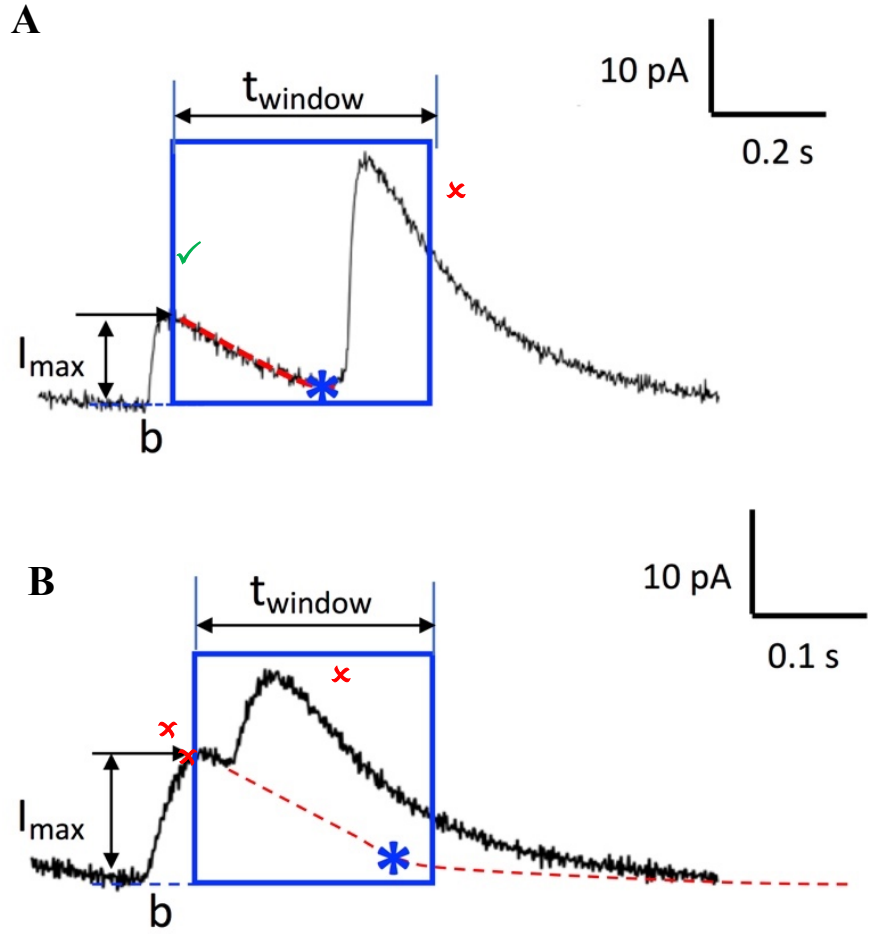

Fig. 2.6. Overlapping spikes rejection criteria

(A) A second spike occurred within a time window set by $t_{\text {window }}=\mathrm{I}_{\operatorname{Imax}}+\tau_{\mathrm{d}} * \ln \left(\mathrm{I}_{\max } / \mathrm{I}_{\min }\right)$ and was hence rejected. The first spike decayed to $3^{*} \tau_{\mathrm{d}}$ indicated by the blue star and was retained for further analysis.

(B) A second spike occurred within the time window set by $t_{\text {window }}=t_{\operatorname{Imax}}+\tau_{d} * \ln \left(I_{\max } / I_{\min }\right)$ and was hence rejected. The first spike did not decay to $3 * \tau_{\mathrm{d}}$ indicated by the blue star and was rejected.
The first step in spike

estimation is the

identification and rejection of

overlapping spikes.

Traditional approaches to

rejecting overlaps rely on

user intervention to define the acceptable extent of overlap,

and, in our experience, often

fail to identify two spikes

located close together in time.

Here we describe a user-

independent algorithm that

detects overlapping spikes

using two criteria.

Criterion 1: For every pair of successive spikes, the second spike (S2) is rejected for analysis if it occurs prior to when the decay of the first spike (S1) is predicted to fall below a minimum current (Imin, typically $1 \mathrm{pA}$ ). The rationale for this criterion is that the first spike has not decayed sufficiently to avoid disrupting the baseline for the second spike. The least-squares fit of the template(s) is used to predict the decay of S1. In the case of a single-exponential decay, the falling phase of a spike is approximately given by, 


$$
f(t)=\operatorname{Imax} \exp \left(-\frac{t}{\tau_{d}}\right)
$$

where, Imax is the peak amplitude determined from the amperometric spike. By setting this function to $I_{\min }$, the minimum acceptable interval until $\mathrm{S} 2$ is given by:

$$
t_{\text {window }}=\tau_{d} \ln \left(\frac{\operatorname{Imax}}{\operatorname{Imin}}\right)(\text { Equation 9) }
$$

For events classified as double exponential, the slower time constant $\tau \mathrm{d} 2$ was used to compute $t_{\text {window. }}$ An $I_{\min }$ of $1 \mathrm{pA}$ was found to be sufficient for rejecting overlaps. Fig. 2.6.(A) and Fig. 2.6.(B) show examples where the second spike is rejected because it falls within $t_{\text {window }}$ of the peak time of the first spike.

Criterion 2: The first spike is rejected for analysis if the second spike occurs less than three times the slower decay time constant $(3 \tau \mathrm{d} 2)$ from the time of the peak of the first spike. The rationale for this criterion is that a duration of three-time constants is sufficient to ensure that the spike time course can be fit with high confidence. In Fig. 2.6.(A) the first spike is accepted because the $3 \tau \mathrm{d} 2$ time point (blue asterisk) occurs before the second spike started. However, in Fig 2.6. (B) the first spike is rejected because another spike occurs before the first spike decays to $3 \tau \mathrm{d} 2$.

\subsubsection{Iterative curve fitting of spikes}

The next step in spike estimation is curve fitting to a spike model. The templates used to detect spikes are a function consisting of a fast-exponential rise followed by a slower exponential decay: 


$$
f(t)=\left(1-\exp \left(-\frac{t}{\tau_{r}}\right)\right) \exp \left(-\frac{t}{\tau_{d}}\right) \quad \text { (Equation 10) }
$$

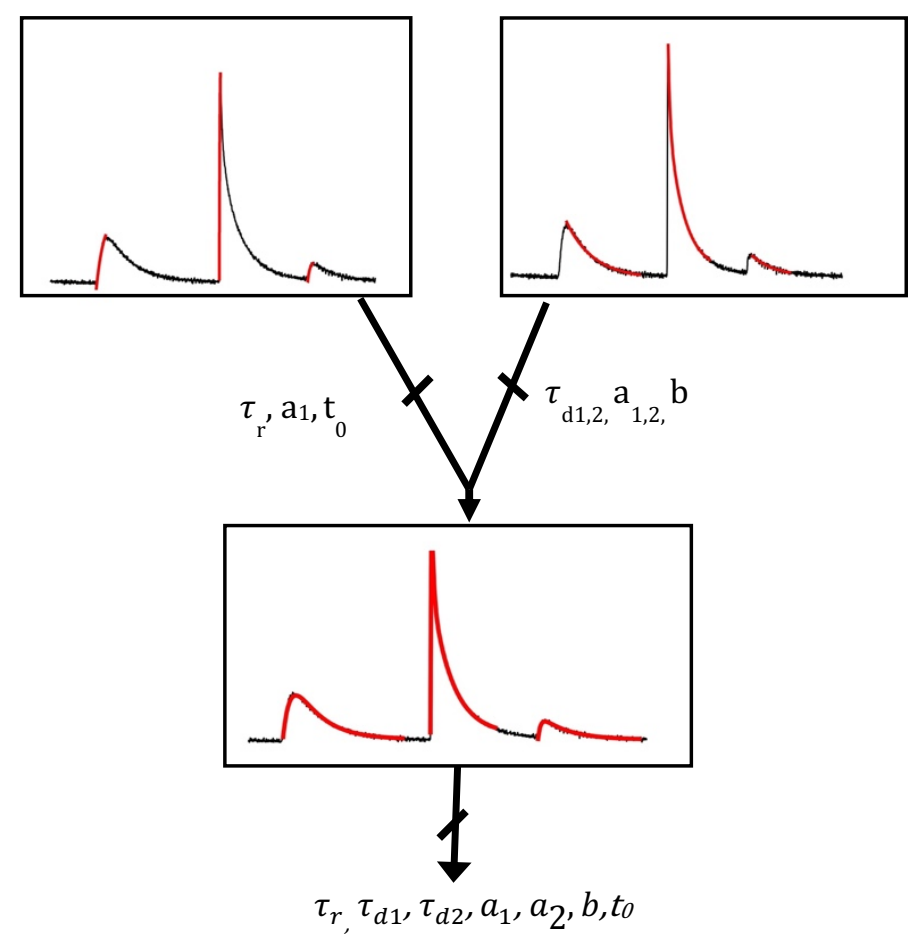

Fig. 2.7. EMF template parameters provide seed values for a two-step non-linear curve fitting routine

Top Left: Rising phases of well separated spikes are fit using the function $\mathrm{a}_{1} *\left(1-\exp ^{(-(\mathrm{t}-\mathrm{t} 0) / \mathrm{rr})}\right)+\mathrm{b}$, Right: Decaying phases of well separated spikes are fit using the function $a_{1} * \exp ^{(-(-t-10) / \mathrm{dd} 1)}$ $+\mathrm{a}_{2} * \exp ^{(-(\mathrm{t}-\mathrm{t} 0) / \mathrm{d} 2)}+\mathrm{b}$. Bottom Parameters obtained from fits in the Top Fig.s seed the function, (1-exp $\left.{ }^{(-(t-t)) / r r)}\right) *\left(a_{1} * \exp ^{(-(t-)}\right.$ $\left.\left.{ }^{\mathrm{t} 0}\right) / \mathrm{d} 11\right)+\mathrm{a}_{2} * \exp ^{(-(\mathrm{t}-\mathrm{t} 0) / \mathrm{d} d 2)}+\mathrm{b}$ to fit entire spikes

Where, $\tau_{\mathrm{r}}$ is the rise time constant and $\tau_{\mathrm{d}}$, is the decay time constants that are selected as representative of "typical" spike time courses. This least-squares fit of the template to the data $(y(t))$ is of the form,

$$
\hat{y}(t)=a f(t)+b \quad(\text { Equation 11) }
$$

Where the amplitude $a$ and baseline / offset $b$ are found using the (non-iterative) least-squares algorithm (Balaji Ramachandran and Gillis, 2018). Our extended 
matched filtering approach fits spikes to a sum of templates with different decaying time constants in the form:

$$
\hat{y}(t)=a_{1} f_{1}(t)+a_{2} f_{2}(t)+b ; t>0 \quad(\text { Equation 12) }
$$

Whereas least-square fits of templates to spikes enables excellent spike identification, adjustments of the time constants are necessary to enable estimation of spike parameters based on precise fits to the data. Since the function has a non-linear dependence on the time constants, an iterative curvefitting approach is needed, and appropriate convergence of the fit is highly sensitive to initial parameter estimates (seed values). Parameters obtained from the least-square template fits were used as seed values in an iterative curve fitting routine (Levenberg, 1944; Marquardt, 1963). The first step was to fit the rising phase of spikes to a function of the form,

$$
\hat{y}(t)_{\text {rise }}=a\left(1-\exp \left(-\frac{(t-t 0)}{\tau_{r}}\right)\right)+b(\text { Equation 13) }
$$

$; \mathrm{t}_{0}<\mathrm{t}<\mathrm{t}_{\text {peak }}$

Subsequently, the decaying phase of the spike is fit to a single or double exponential, depending on the classification. For a double-exponential fit the decaying phase is of the form

$$
\hat{y}(t)_{\text {decay }}=a_{1} \exp \left(-\frac{(t-t 0)}{\tau_{d 1}}\right)+a_{2} \exp \left(-\frac{(t-t 0)}{\tau_{d 2}}\right)+b(\text { Equation 14) }
$$

$; \mathrm{t}>\mathrm{t}_{\text {peak }}$

Examples of fits to the rising and decaying phases are shown in the top of Fig. 2.7. In the final fitting step, entire spikes are fit by seeding output parameters from the separate rising and decaying fits to the function described as (for the double-exponential case): 


$$
\hat{y}(t)=\left(1-\exp \left(-\frac{(t-t 0)}{\tau_{r}}\right)\right)\left(a_{1} \exp \left(-\frac{(t-t 0)}{\tau_{d 1}}\right)+a_{2} \exp \left(-\frac{(t-t 0)}{\tau_{d 2}}\right)\right)+b
$$

(Equation 15)

$$
; \mathrm{t}_{0}<\mathrm{t}<\mathrm{t}_{\text {peak }}+3 * \tau_{\mathrm{d} 2}
$$

Seed values for $\tau_{\mathrm{r}}$, and $\mathrm{t}_{0}$ are from the rising phase fit whereas $\mathrm{a}_{1,2}, \mathrm{~b}$, and $\tau_{\mathrm{d} 1,2}$ are from the decaying phase fit. Fig. 2.7. Bottom depicts typical examples where excellent fits to the entire spike are obtained.

After the fit, the entire time course, amplitude and area of each spike is reduced to either three parameters (single exponential decay: $\tau_{\mathrm{r}}, \mathrm{a}, \tau_{\mathrm{d}}$ ) or five parameters (double exponential decay: $\tau_{\mathrm{r}}, \mathrm{a}_{1}, \mathrm{a}_{2}, \tau_{\mathrm{d} 1}, \tau_{\mathrm{d} 2}$ ). These parameters are then used to calculate the traditional spike parameters including the area under the curve or charge $(\mathrm{Q})$, full width half maximum $\left(\mathrm{t}_{50}\right)$ and peak amplitude $\left(\mathrm{I}_{\max }\right)$. An idealized spike is reconstructed using time course parameters, and roots of the spike function for a $y$ value of $I_{\max } / 2$ are located on either side of the peak and the time difference between these roots is calculated as $t_{50}$. The peak of the idealized spike is computed as $I_{\max }$. The spike function is integrated to compute Q. In addition, arbitrary measures of the spike rising phase such as the $25 \%$ to $75 \%$ rise time can be calculated. 


\subsubsection{Baseline identification and pre-spike foot analysis}

The next step of the algorithm is to estimate parameters describing the pre-spike foot. Accurately identifying and analyzing pre-spike foot signals is dependent on the accurate determination of pre-spike baseline current. We devised an algorithm to compute pre-spike baseline current as an extension of our template-based matched filtering approach used for spike detection. As templates are leastsquares fit to each segment of data, a criterion score is created at each time point that is the ratio of the amplitude of the template fit divided by the standard error of the fit to the data segment

$$
\text { criterion score }=\frac{a}{S E} \quad(\text { Equation 16) }
$$

In the case of fits to a sum of two templates with fast and slow decay time constants, the criterion score is given by:

$$
\text { criterion score }=\frac{\left(a_{1}+a_{2}\right)}{S E} \quad(\text { Equation 17) }
$$

where a1 and a 2 are the least-squares amplitudes found for the two decaying phases. Peaks in the criterion score that exceed a threshold identify spikes during the detection phase. On the other hand, a criterion score of zero occurs when the data segment is flat, i.e., the amplitude of a spike fit is zero, indicating a potential baseline segment. Fig. 2.8. depicts a typical 
amperometric spike

with corresponding

criterion scores. Starting

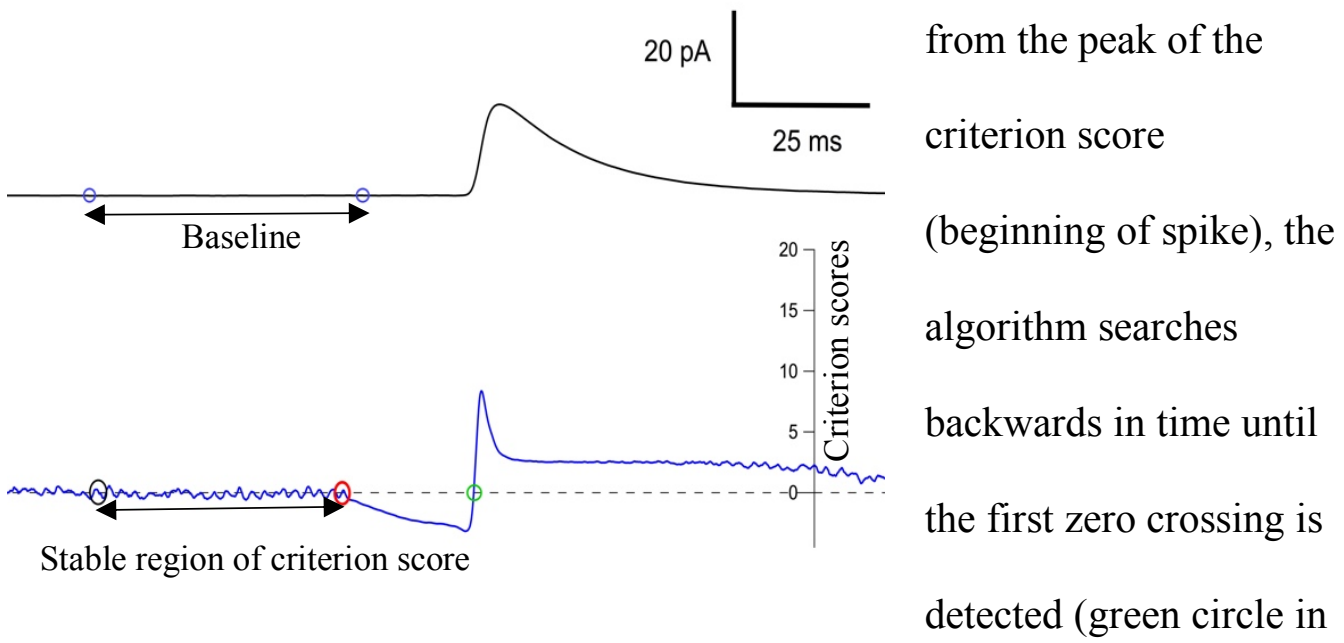

Fig. 2.8. Mf criterion scores can be used to determine baseline

Fig. 2.8.). This zero

Top shows an amperometric trace (black). Bottom shows the corresponding MF criterion score trace(blue). In the region preceding the criterion score peak (spike start time), first zero is shown by a green circle, positive zero crossing of the criterion score (second zero) is shown by the red circle. The criterion score is stable in the region enclosed by the red and black circles. The top trace shows that the baseline is computed in the stable region preceding the second zero, in between the corresponding two blue circles.

crossing occurs as the template and the data first begin to align. This is preceded in time by a negative criterion score

where the template decaying phase aligns with the spike rising phase, leading to a negative correlation. The algorithm continues to search backward in time until the second zero crossing of the criterion score occurs (red circle in Fig. 2.8), which normally indicates the region where the baseline is flat. In order to test for a stable baseline, the algorithm determines the standard deviation of the criterion score for a segment of data preceding the second zero crossing with a duration equal to that of the template (line segment in Fig. 2.8.). If the standard deviation of this 
segment is less than or equal to 0.05 , this segment of the amperometric data is averaged to give the baseline value. If the standard deviation is greater to 0.05 the segment is moved backward in time point by point until a stable baseline is identified. This procedure for determining the pre-spike baseline is repeated for each spike. The start time for a pre-spike foot is identified as when the signal rises two standard deviations above the baseline value (Mosharov and Sulzer, 2005; Zhao et al., 2013) with the current standard deviation measured from the baseline data segment. The end of the foot signal is defined as $\mathrm{t} 0$ determined from the iterative curve fit of the corresponding spike. This is the point where the exponential rise of the spike begins (maximum slope), and hence signifies the end of the foot signal.

† Reproduced from (Balaji Ramachandran and Gillis, 2018) 


\section{Chapter 3}

\section{A MATCHED-FILTER ALGORITHM TO DETECT AMPEROMETRIC SPIKES RESULTING FROM QUANTAL SECRETION †}

\subsection{Automated detection of amperometric spikes}

As discussed in Chapter 1 (section 1.4), manual detection of amperometric spikes is time-consuming and error prone. Hence there is a need for automation to reliably locate amperometric events. The major considerations in amperometric spike detection are (a) diverse amplitudes (b) variable time constants (c) a wavering baseline and (d) closely successive overlapping spikes are ambiguous to resolve. Existing approaches (Friedrich and Ashery, 2010; Mosharov and Sulzer, 2005; Segura et al., 2000) have several problems including bias against smaller/slower spikes, misidentification of two overlapping spikes as one and reliance on user-input parameters for filter settings. As described in Chapter 2 (section 2.3), we demonstrated a matchedfilter algorithm to detect spikes without using any traditional filters and minimal user-intervention. In brief, a library of spike templates is used to perform least-squares fitting on the data using variable time constants, a twothreshold approach is applied to the detection criterion scores to identify spikes, receiver operating characteristic (ROC) plots are used to quantify detection performance and spike parameters including offset time $\left(\mathrm{t}_{0}\right)$, rise time constant $\left(\tau_{\mathrm{r}}\right)$, decay time 
A

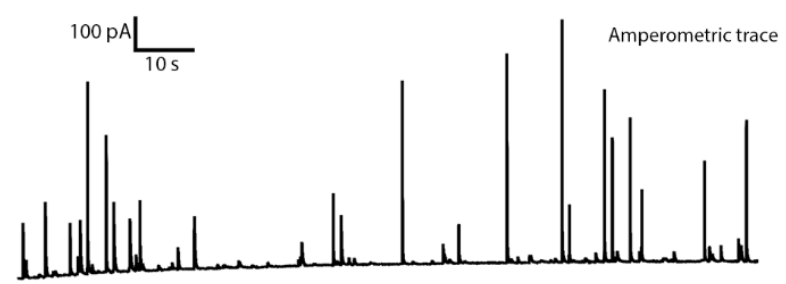

B

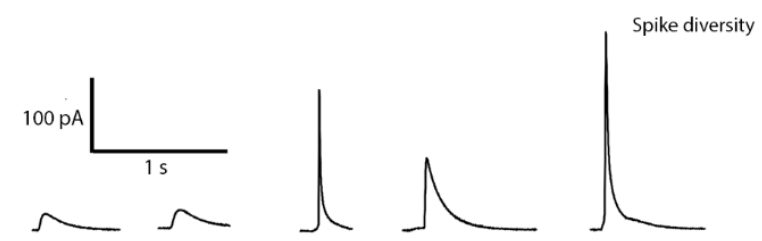

C

$100 \mathrm{pAl}$
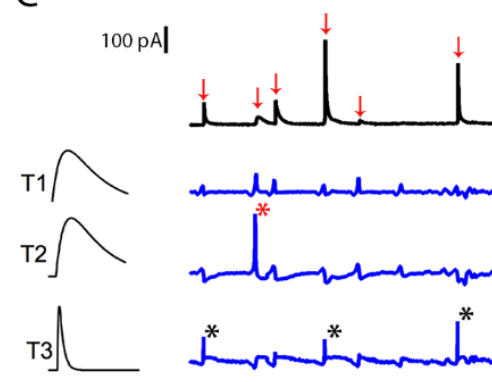

Amperometric trace
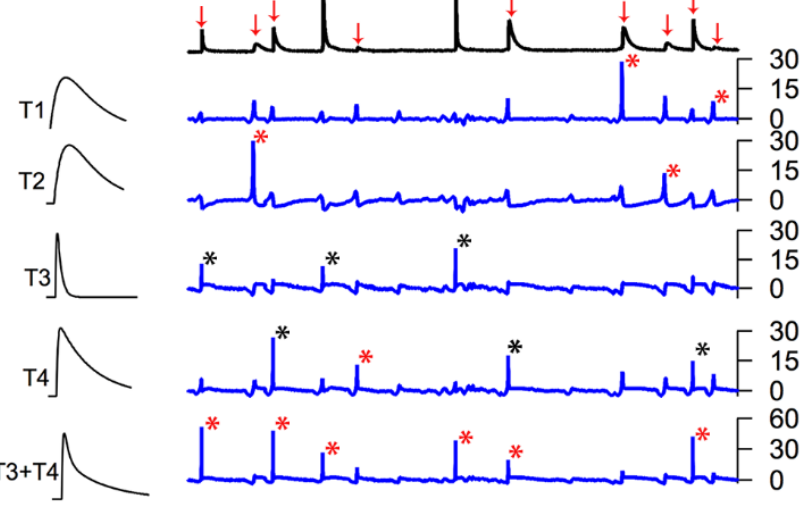

Fig. 3.1. The heterogeneity of spike time courses necessitates the use of multiple templates for detection

(A) A sample amperometric recording (B) Samples of amperometric spikes illustrating the heterogeneity of time courses and amplitudes. (C) Solid black trace: sample data segment with spikes. Blue traces: criterion scores for each of the templates depicted on the left. The red stars indicate the template with the highest criterion score for each spike. The black stars represent the highest-scoring template when using only single exponentially decaying templates, but where the sum of two templates $(\mathrm{T} 3+\mathrm{T} 4)$ approach resulted in a higher score (red stars). constants $\left(\tau_{\mathrm{d} 1}, \tau_{\mathrm{d} 2}\right)$, decay

amplitudes $\left(\mathrm{a}_{1}, \mathrm{a}_{2}\right)$, and

baselines (b) are extracted

for use as initial seed values

to iteratively fit and

estimate spikes in Chapter

4. The following sections

describe the results of the

matched-filter spike

detection approach.

\subsubsection{Multiple}

templates are required to

detect exocytotic spikes

with varying time courses

As illustrated in Fig. 3.1.A,

$\mathrm{B}$, exocytotic spikes in

chromaffin cells vary

considerably in time course,

therefore a single template

is insufficient to detect both

slow and fast spikes. We

therefore chose four templates that represent a range of spike dynamics found in chromaffin cells (Fig 3.1.C, left). Template 1 has a slow rise and slow decay, 
A

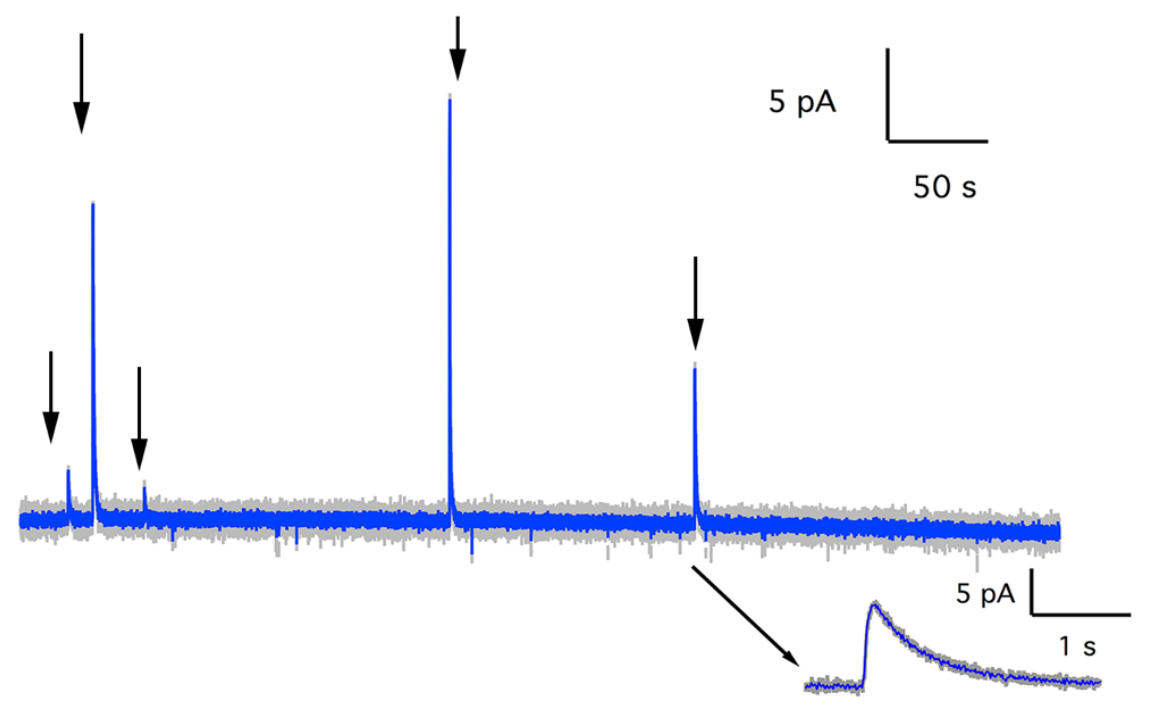

过

B

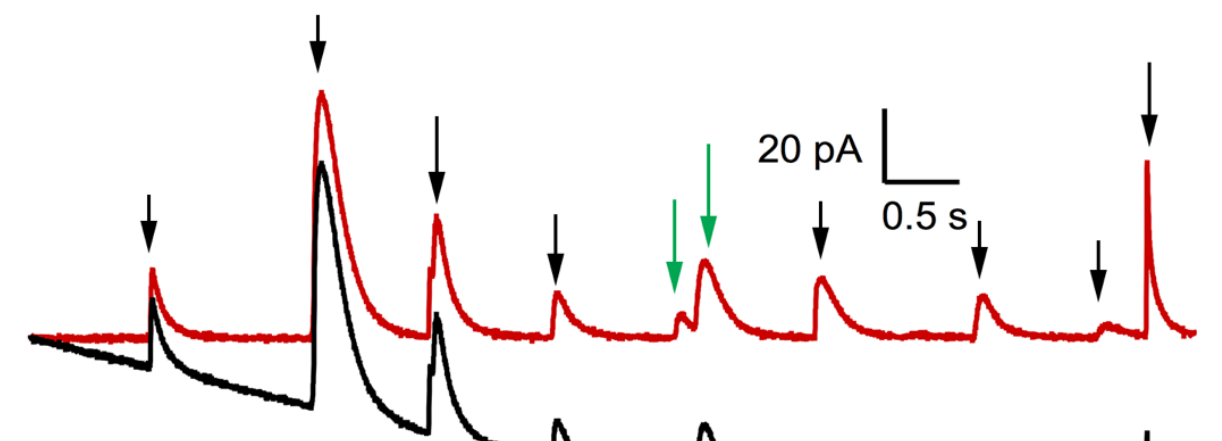

Fig. 3.2. Filtering and baseline drift do not affect detection performance

(A) Top: Amperometric trace sampled at $10 \mathrm{kHz}$ (gray) and decimated to 1 $\mathrm{kHz}$ (blue). Black arrows point to true spikes.

Bottom: Criterion scores corresponding to the unfiltered (dashed gray) and the decimated (dashed blue) data. Inset expanded single spike shows the original traces (top) and the criterion scores (bottom).

Red dotted line is the criterion threshold of 2.6.

(B) Top: Original amperometric trace (red) and trace with a baseline slope of -10pA/s (black). All the arrows point to true spikes. Green arrows point to overlapping spikes.

Bottom: Criterion scores for original trace (dashed red) and trace with $-10 \mathrm{pA} / \mathrm{s}$ baseline slope (dashed black). Blue dotted line is the criterion threshold of 2.6 . 
template 2 has a slow rise and intermediate decay time, template 3 has a fast rise and fast decay and template 4 has a fast rise and a slow decay. Finally, a sum of templates was used to fit spikes with double exponential decays. Fig 13C (blue traces) presents criterion scores for the four templates calculated from a sample trace. Note that, in contrast to detection of spontaneous post-synaptic events (Clements and Bekkers, 1997), no individual template produces criterion score peaks that enable resolution of all of the spikes. The red star indicates the template with the highest criterion score for each spike. Thus use of multiple templates is not only essential for exocytotic spike detection, but also leads to classification of spikes into broad categories defined by the best-match template (van Kempen et al., 2011).

\subsubsection{Use of two thresholds reduces false-positives}

A spike is detected when the criterion score exceeds a threshold, and the time when the score reaches a peak identifies the start time of the detected spike. The criterion score then declines as the template is moved past the start-time of the spike, subsequently rising again as it approaches the next spike. Therefore, the

algorithm needs to reset the search for a new spike upon a decline in the criterion score. The simplest approach is to reset the search when the criterion score drops below the same threshold used to detect the spike (Clements and Bekkers, 1997). However, this can lead to false positives. 
Fig. 20(A) shows an

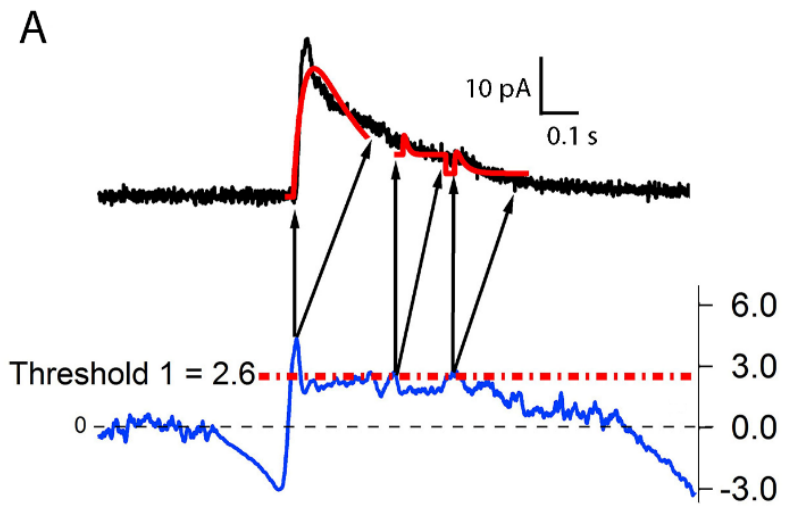

B
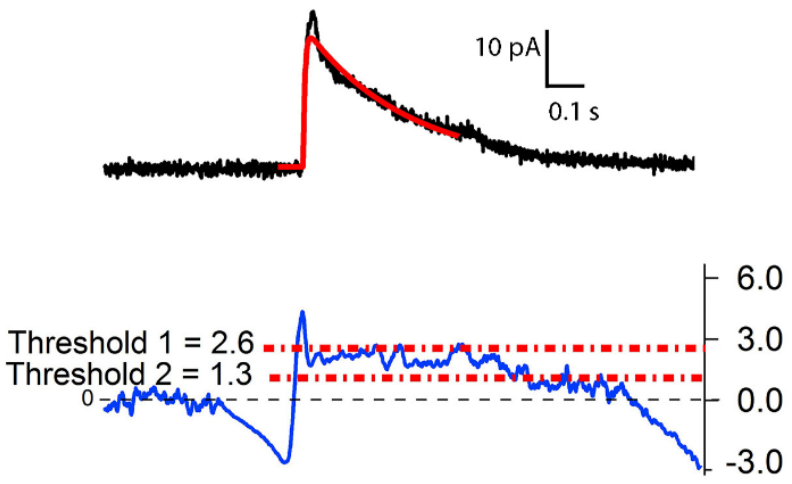

Fig. 3.3. A two-threshold approach reduces false positives

(A) A single threshold algorithm can produce false positives as the criterion score fluctuates above and below the threshold on the falling phase of a spike. (B) False positives are avoided if the criterion score must fall below a second, lower threshold before initiating a search for the next spike. Black: data trace. Red: least-squares fits determined at the peaks of the criterion scores Blue: Criterion scores example trace containing a

single spike, yet two

additional spurious spikes

are detected (red traces) as

the criterion score fluctuates

around the threshold value

as the template is moved

across the declining phase of

the exocytotic spike. In

order to reduce false

positives, we used two

thresholds. A higher

threshold is applied to detect

a spike, but then the criterion

score must decline below a

lower threshold before the

search for a new spike is

initiated. Fig. 20(B) shows

the same spike from Fig. 20(A) where use of the second lower threshold prevents the detection of spurious spikes because the criterion score does not drop below the lower threshold and then rise above the higher threshold until the next spike is 
encountered. We found that setting the lower threshold to $50 \%$ of the value of the higher threshold was sufficient to avoid false positives.

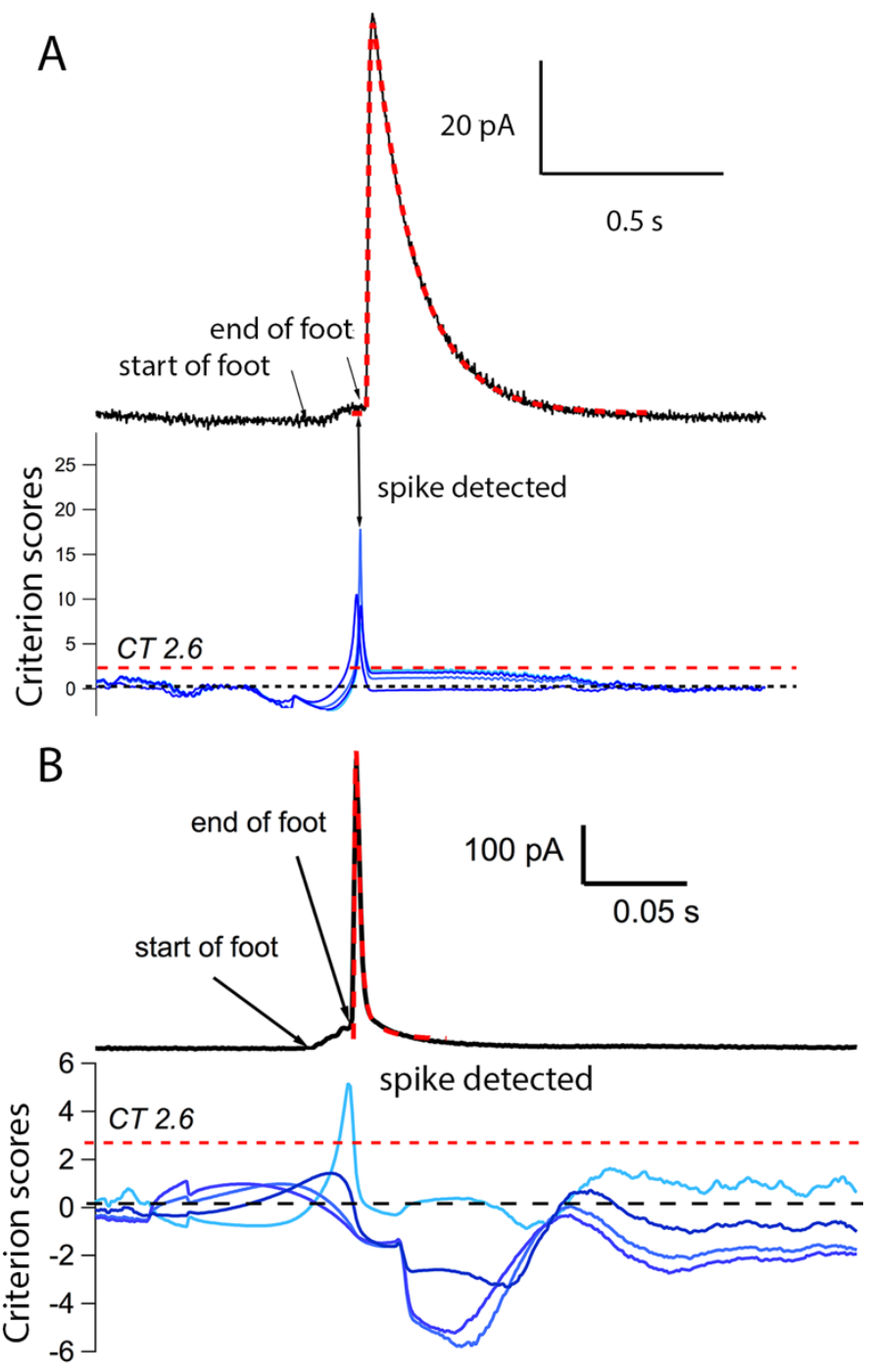

Fig. 3.4. Foot signals do not affect detection of spikes

(A) Top: Amperometric trace (black) preceded by a ramp + plateau type foot signal with the MF fit (red dotted). Bottom: Criterion scores of four templates (varying shades of blue). (B) Top: Amperometric trace (black) preceded by a ramp type foot signal with the MF fit (red dotted). Bottom: Criterion scores of four templates (varying shades
3.1.3. The

matched-filter approach

is not very sensitive to

filtering and can detect

spikes with a sloping

baseline.

The matched filter

approach uses hundreds of

recorded data values to

calculate each criterion

score and is therefore not

highly sensitive to random

noise fluctuations resulting

from insufficient filtering

of the data. Fig.

3.2.(A) presents

criterion scores for

template 3 applied to a

raw data trace compared to the same data trace after the 10:1 decimation filtering that we normally use. Note that each of the five amperometric spikes have clearly defined criterion-score peaks even for a $\sim 10$-fold increase in bandwidth 
above the minimum necessary to resolve fast spikes. Nevertheless, it is recommended that traces be filtered at a cutoff frequency no higher than necessary to preserve spike dynamics in order to minimize noise and potential false positives. In addition, using a lower sampling rate speeds the detection algorithm because fewer points are used in the calculation. The matched filter approach is also not very sensitive to a sloping baseline because, unlike amplitude-threshold algorithms, it does not rely on precise identification of baseline values. Fig. 3.2.(B) presents criterion scores for a data trace before and after adding a sloping offset to simulate an extreme case of a sloping baseline. Note this trace also shows an example where both spikes in an overlapping pair are clearly detected.

\subsubsection{The matched-filter approach detects amperometric spikes with pre-spike features.}

Spike events resulting from exocytosis are often preceded by small-amplitude features that result from efflux of transmitter through a narrow fusion pore (Alvarez de Toledo et al., 1993; Chow et al., 1992b). Fig. 3.4. presents examples demonstrating successful identification of spikes preceded by either plateauing (Fig. 3.4(A)) or ramping (Fig. 3.4(B)) pre-spike features. Note that the pre-spike features themselves are not incorrectly identified as "false positive" spikes because they do not have the characteristic shapes represented in the spike template library. 


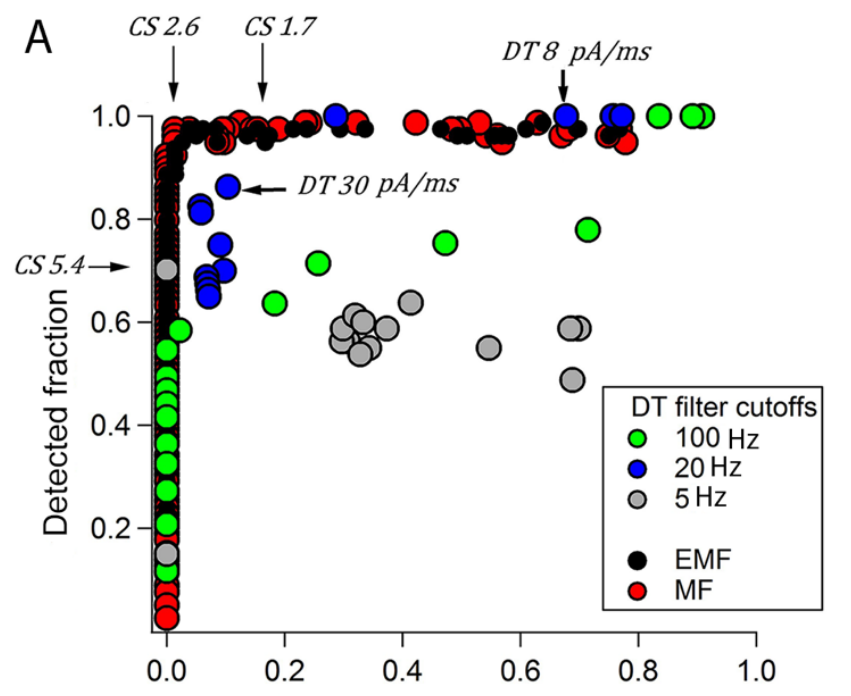

B

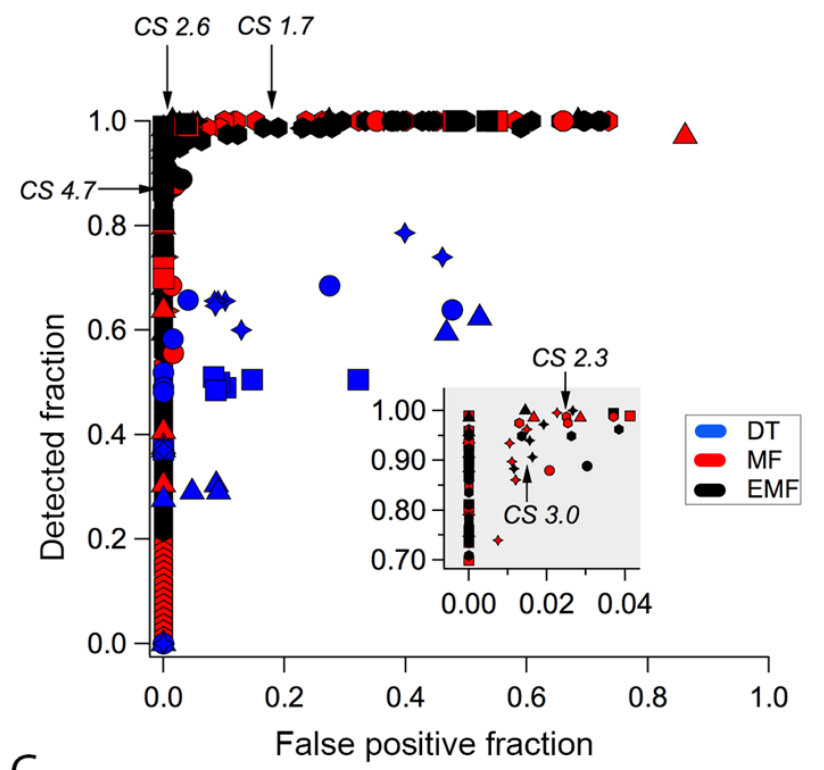

C
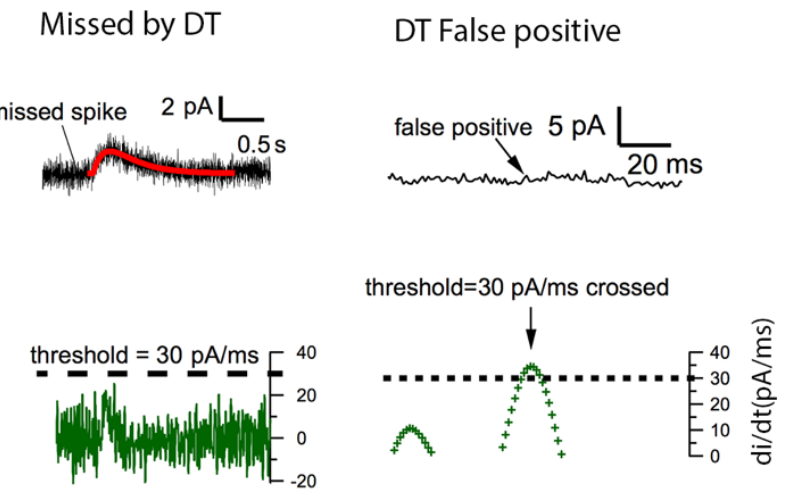

Fig. 3.5. The MF algorithm outperforms the DT approach in bovine chromaffin cell recordings

(A) ROC plot for a recording comparing the DT algorithm with several filter settings with the MF and EMF approaches. Several criterion scores (CS) are indicated by arrows on the plot. (B) ROC plot for five recordings comparing the performance of spike detection algorithms. Blue: DT, red: $M F$, black: $E M F$. Each data set is represented by a unique symbol. Inset: expanded view near the ideal corner. (C) Examples of missed events and false positives using the DT approach. Black traces: section of amperometric data showing missed spike (left) and false positive (right), Red trace: MF fit to spike detected by MF algorithm, green trace: current derivative (dI/dt)

However, if a pre-spike event

displays both a rising and falling

phase preceding a second larger

spike event, the first event may

be identified as a separate spike.

In these cases, it is often unclear

whether the first event is a foot

signal or a small, independent

spike. 
A

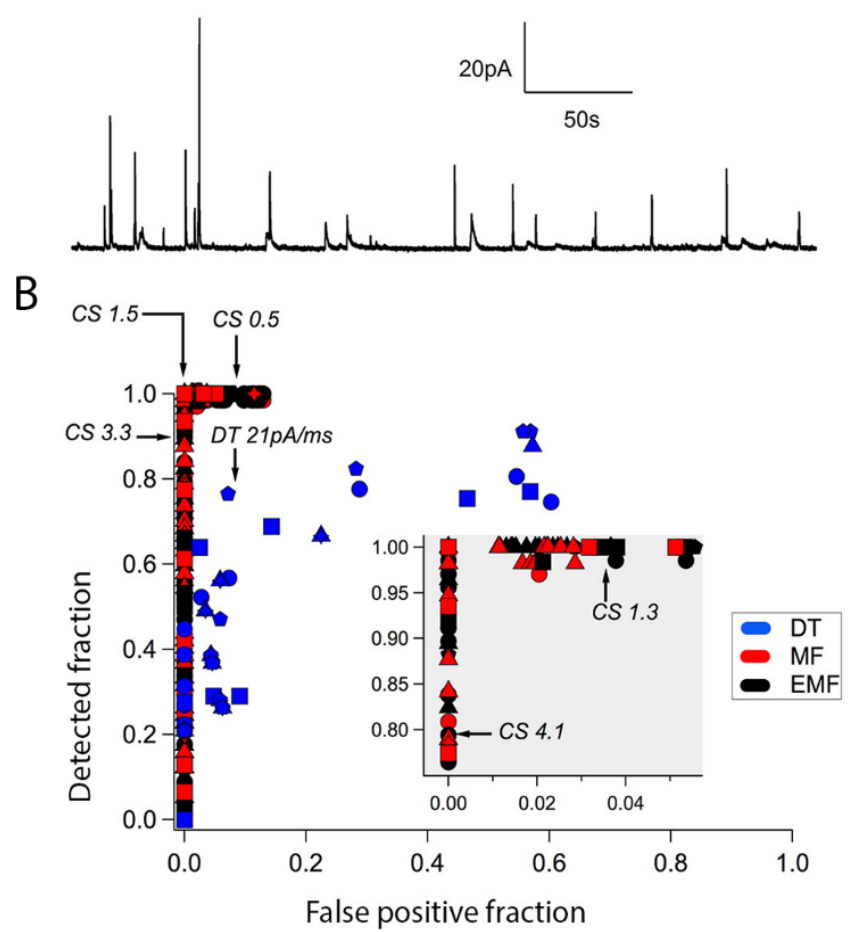

C

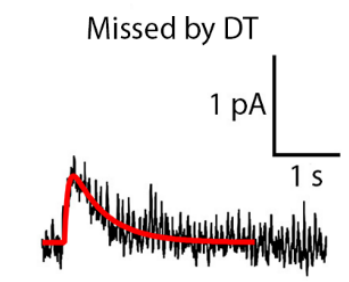

DT False positive
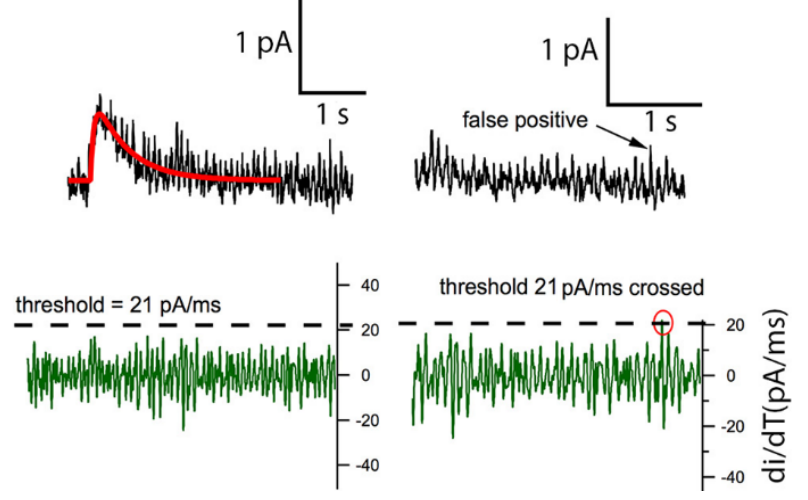

Fig. 3.6. The MF algorithm outperforms the DT approach in bovine chromaffin cell recordings

(A) Amperometric trace (B) Receiver Operating

Characteristic plots comparing the performance of spike detection algorithms for five recordings. Blue: DT, red: $M F$, black: EMF. Each data set is represented by a unique symbol. Inset: expanded view near the ideal corner. (C) Examples of missed events and false positives using the DT approach. Black traces: section of amperometric data showing a missed spike (left) and a false positive (right), Red trace: MF fit to spike detected by MF algorithm, green trace: current derivative (dI/dt).

\subsubsection{Extending}

\section{the matched-filtering}

approach to detect spikes

\author{
with a sum of two \\ templates
}

Some exocytotic spikes

decay in a double

exponential fashion, having a

first fast decay followed by a second slower decay (Segura

et al., 2000). We extended the matched-filter algorithm to produce least-square fits with a sum of two templates: $f_{1}(t)$ and $f_{2}(t)$. The fit is therefore of the form:

$$
\begin{aligned}
\hat{y}(t)=a_{1} f_{1}(t) & \\
& +a_{2} f_{2}(t) \\
& +b \\
& =\mathrm{X} * \beta
\end{aligned}
$$


where $\boldsymbol{X}$ is the template matrix given by:

$$
X=\left[f_{1}(t)\left|f_{2}(t)\right| \mathbf{1}\right]
$$

and $\beta$ is the parameter vector given by:

$$
\beta=\left[\begin{array}{c}
a_{1} \\
a_{2} \\
b
\end{array}\right]
$$

The Least Squares solution for the parameter vector is therefore given by:

$$
\hat{\beta}=\left[X^{T} X\right]^{-1} X^{T} y
$$

Thus, the LS fits to a sum of two templates are calculated for each possible starting point of a segment of data in the same manner that fits are calculated for each individual template. The criterion score for a double-template fit is calculated as:

$$
\text { criterion score }=\frac{\left(a_{1}+a_{2}\right)}{S E}
$$

Note that fitting data with a sum of two templates is preferable to creating a new template with a double-exponential decay, because it allows the relative amplitudes of the two exponential components to be freely adjusted to obtain a better fit to the spike. As noted by the red stars in Fig. 3.1(C), the double-template fit can often produce higher criterion scores (better fits) than use of a single template. The double-template fit was accepted as the best fit, and thus classifies the spike in the double-exponential category, only if 1) neither amplitude is 
negative, and 2) the smaller amplitude is at least $10 \%$ as large as the larger amplitude.

\subsubsection{The matched-filter approach outperforms the derivative- threshold approach}

Amperometric recordings from bovine and mouse chromaffin cells were analyzed to compare the Matched Filter (MF) and Extended Matched Filter (EMF, doubletemplate) approaches with the Derivative Threshold (DT) approach. The performance of these approaches for detecting spikes was measured using Receiver Operating Characteristic plots described in the Methods section. In brief, reducing the threshold for detection (criterion score for MF and EMF, derivative in $p A / m s$ for DT) results in detection of more spikes (thus higher sensitivity), but will eventually result in a greater number of false positives (thus lower specificity). An optimal algorithm using an ideal threshold will result in 100\% detection with $0 \%$ false positives, i.e., the upper left corner of an ROC plot. Fig. 3.5(A) presents a representative ROC plot for a recording from a bovine chromaffin cell containing 80 spikes. The performance of the MF (red) and EMF (black) approaches are similar and are contrasted to that of the DT approach with several different settings of low-pass filtering. The MF approach detects $100 \%$ of the spikes for $2 \%$ false positive fraction (criterion score $=2.6$ ). The performance of the DT approach depends on the filtering cutoff frequency (Fig. 3.5(A)). 


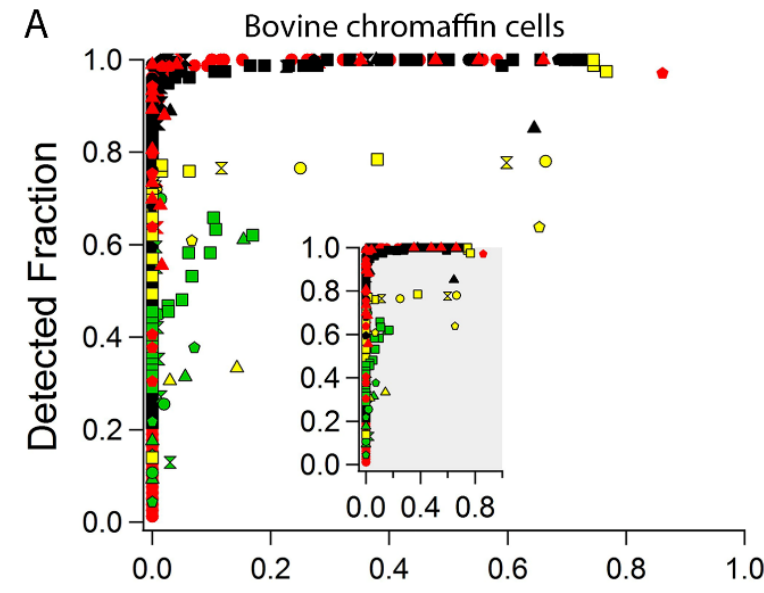

False positive fraction

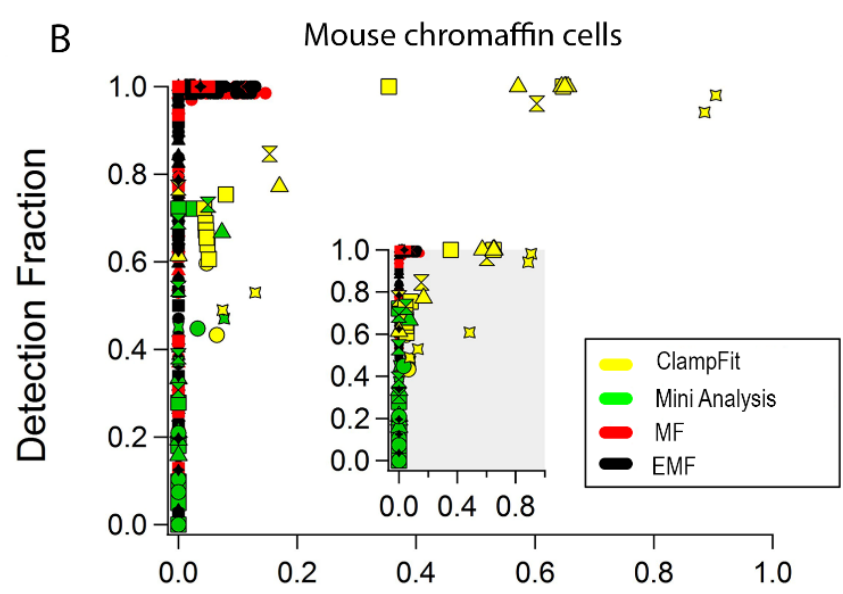

False positive fraction

C

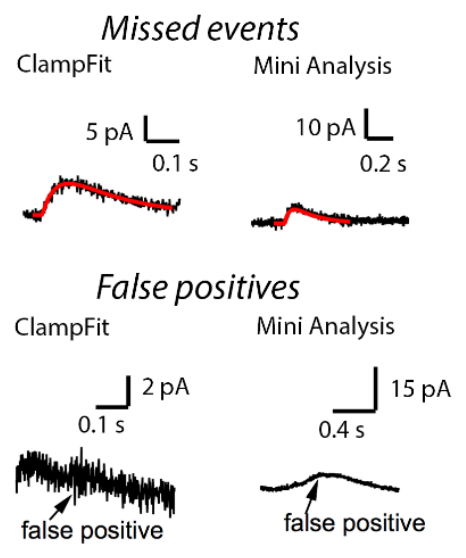

D

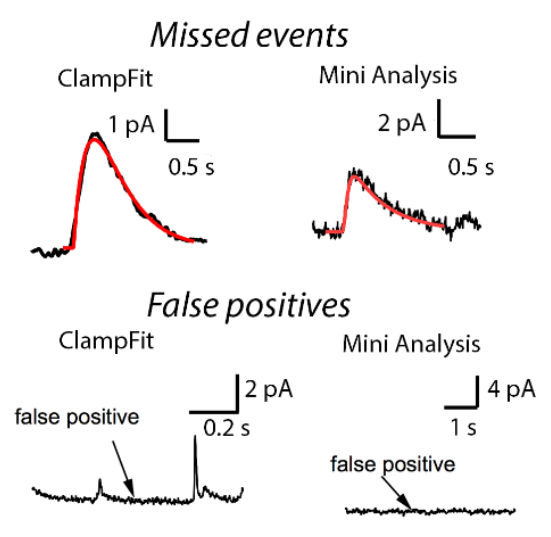

Fig. 3.7. Mf outperforms clampfit and minianalysis

For bovine (A) and mouse (B) chromaffin cells. Receiver Operating Characteristic plots comparing the performance of spike detection algorithms for five amperometric recordings. MF (red), EMF (black), ClampFit (yellow) and MiniAnalysis (green) are shown. Each data set is represented by a unique symbol Inset: Expanded view of ideal corner. Spikes missed, and false positives detected by ClampFit and MiniAnalysis are shown for bovine (C) and mouse (D) chromaffin cell data. Red trace: MF fit to spike detected by MF algorithm and mouse (D) chromaffin cell data. Red trace: MF fit to spike detected by MF algorithm

Nevertheless, even at the best cutoff frequency $(20 \mathrm{~Hz})$, DT detects only $60 \%$ of the spikes for a $2 \%$ false positive fraction (criterion score $=30 \mathrm{pA} / \mathrm{ms}$ ).

Amperometric events are missed with DT even when the threshold was lowered to the point where the false-positive fraction exceeds $10 \%$. Missed events tend to be smaller and slower, as shown in examples in Fig. 3.5.(C). On the other hand, 
rapid fluctuations in the signal are sometimes misclassified as spikes using DT. Fig. 3.5(B) presents ROC plots for five bovine chromaffin cell recordings that demonstrate similar characteristics. In mouse chromaffin cells (Fig. 3.6) MF and EMF could detect $100 \%$ of the spikes for $0 \%$ false positive detection (criterion score $=1.7)$ whereas DT could detect $60 \%$ of the spikes for the same false positive fraction (filter setting, DT=21 pA/s).

\subsubsection{The matched-filter approach outperforms commercial event- detection software}

A similar ROC analysis was employed to compare the performance of MF algorithm to commercial applications primarily intended for analysis of spontaneous post-synaptic events: MiniAnalysis (Synaptosoft, Fort Lee, NJ, USA) and ClampFit (Molecular Devices, Sunnyvale, CA, USA).

When the threshold for these methods is set to a value that results in $\sim 2 \%$ false positives, the MiniAnalysis and ClampFit applications only detect $45 \%$ (at threshold $=3$ ) and $70 \%$ (at threshold $=4$ ), respectively, of the spikes in bovine chromaffin cells (Fig. 3.7(A)). In contrast, the MF or EMF approaches are able to detect $100 \%$ of the spikes for $0 \%$ false positives (threshold $=2.6$ ). In mouse cells, MiniAnalysis and ClampFit detect 70\% (at threshold $=3$ ) and 75\% (at threshold $=$ 3) of the spikes for zero false positives (Fig. 3.7 (B)). Thus, the MF approach emerged as the superior method among all available detection applications. Examples of missed spikes and false positives for MiniAnalysis and ClampFit are depicted in Fig. 3.7(C, D). 
The conditions for detection such as filtering, and detection threshold for each software program were chosen to ensure that the detection performance was optimal. For example, the Derivative Threshold approach was tested at the optimal smoothing frequency; The same template library was used to apply ClampFit software as the MF algorithm. However, the experimenter did not have access to all controls in commercial software and this affects the scope of the ROC plot performance comparison.

\subsection{Discussion}

A MF approach was applied for the detection of exocytotic events in amperometric recordings. The performance of this method is superior to existing commercial and user-community applications, as demonstrated by ROC plots. The MF technique provides two main advantages. First, it eliminates the need for low pass filtering the data for noise reduction. DT approaches rely on data smoothing both before and after computing the derivative of the original recording and the best choice of filter setting depends on the signal-to-noise ratio of the recording (Gómez et al., 2002; Mosharov and Sulzer, 2005). Excessive low-pass filtering of data can cause the algorithm to overlook actual spikes whereas inadequate smoothing can increase false positive detection due to noisy transients (Fig. 3.4.). In addition, the setting of the derivative threshold that maximizes sensitivity and selectivity depends on the low-pass filter setting. Hence it is not straightforward to choose low pass filter settings. 
A second advantage of the MF technique is that detection bias is explicit in the choice of the templates to be included in the library. The MF method uses information from both the rising and falling phases of the spike to enable detection that is rationally biased by the choices made in constructing the template library. In contrast, the DT approach relies on a rapidly rising phase of the spike to enable detection, and therefore introduces a bias against detecting slowly rising spikes.

An alternative approach to detect exocytotic spikes is to subtract the time-varying baseline from the signal and then detect spikes that exceed amplitude thresholds (Friedrich and Ashery, 2010). However, tracking the time-varying baseline with pA precision requires that the baseline be stable over the averaging time window and relies on user input of the noise level. Also, in an amplitude-based algorithm small-amplitude flickers may be mistaken as spikes (Friedrich and Ashery, 2010) whereas our approach rejects such flickers because their time courses do not match the templates. Avoiding false positives inevitably makes amplitude-based algorithms more biased against small-amplitude events than template-based algorithms.

All reasonable efforts were made to reduce bias in comparing algorithms. Events were classified as "hits" or "misses" (false positives) using automated criteria described in Methods. User-adjustable parameters were selected for each algorithm that maximized performance. Nevertheless, there is the possibility that 
bias can be introduced by the user in the application of the various software packages.

Selection of an appropriate template duration is important for the algorithm to function appropriately. Use of a long-duration template makes it difficult to resolve spikes closely spaced in time whereas a brief template poses the risk of increasing the false positive fraction. We found that a template size of $\sim 200 \mathrm{~ms}$ is optimal for bovine and mouse chromaffin cell recordings. The short pedestal that precedes the template is also critical for high-performance detection, and we found a 20 ms pedestal works well.

Template libraries need to be chosen for the specific biological preparation and the type of event one wants to detect. We selected the time constants for the rising and falling phases for bovine versus mouse chromaffin cells after looking for clusters of time constants in exponential fits to sample data sets. Development of semi-automated methods for creating template libraries would be a useful extension of the approach but is beyond the scope of this study.

The identification of the template that produces the highest criterion score (Fig. 3.1(C) can be used to classify events into categories that describe the nature of the fusion event (e.g., fast versus slow opening of the fusion pore). Addition of new templates may be useful to identify and classify other types of fusion events such as kiss and run (stand-alone feet). 
It should be noted that the Least Squares fitting that is central to this approach is computationally efficient because it is a closed-form solution, unlike iterative curve fitting which is necessary to solve for fitting parameters that are not linearly related to the signal, such as the exponential time constants.

This work concentrates on computationally efficient detection of spikes; however, this is usually followed by estimation of relevant spike parameters such as charge (area), duration (FWHM) and the duration and charge of the "foot signal" that often precedes the spike. One approach to quantify a spike is to fit it with an exponential time course similar to the templates we used but allow the time constants to vary during an iterative fitting process. The MF approach is well suited to provide "seed" values from the LS fit template with the highest criterion score for iterative curve fitting, including the start time, amplitude and time constants.

The EMF approach to fit data to a sum of two templates did not perform substantially better to detect spikes than the simpler approach based on a single template. Nevertheless, this approach performed equal to MF approach for detection and also provides excellent seed values enable efficient fitting of spikes with double-exponential decays during the parameter estimation phase (data not shown). 


\subsection{Conclusions}

Our implementation of the MF algorithm is a highly specific and sensitive amperometric spike detection approach. MF outperforms the classic approach of Derivative Threshold (DT) as well as other commercial and user-community applications such as ClampFit and MiniAnalysis. We thank Dr. Kevin Currie at Vanderbilt for providing amperometric recordings from mouse chromaffin cells.

† Reproduced from (Balaji Ramachandran and Gillis, 2018) from Section 3.1.1 onwards 


\title{
Chapter 4
}

\section{ESTIMATING AMPEROMETRIC SPIKE PARAMETERS RESULTING FROM QUANTAL EXOCYTOSIS USING CURVE FITTING SEEDED BY A MATCHED-FILTER}

\begin{abstract}
ALGORITHM

\subsection{Need for spike and pre-spike foot signal estimation}

Single-cell amperometry is a powerful technique for studying exocytosis. In this method, electrochemical microelectrodes placed directly adjacent to the cell membrane measure neurotransmitter released from individual vesicles as "spikes" or bursts of amperometric current as the transmitter is oxidized on the surfact of the electrode (Wightman et al., 1991). Close examination of these events reveals that slow release of transmitter through a nanometer-scale fusion pore produces a pre-spike foot signal (Chow et al., 1992a; de Toledo et al., 1993a) that is followed by a rapid rising phase as the fusion pore expands. The spike decays in a single or double-exponential manner as the contents of the vesicle or emptied or as release is terminated upon reclosure of the release pathway. Therefore, the temporal dynamics of amperometric spikes can be used to understand the release process from individual vesicles in great detail and to determine how the release process is modified by drugs, second messengers, and perturbation of proteins that participate in the fusion and release process (Borges et al., 2006) for a review).
\end{abstract}

The first step in amperometric recording analysis is spike detection, which is followed by estimation of parameters. In our earlier work, we described a matched filter (MF) template-based spike-detection algorithm that detected $\sim 97 \%$ of manually identified spikes for a mere $\sim 2 \%$ false positive rate (Balaji Ramachandran and Gillis, 2018). In 
brief, this algorithm uses a least-squares approach to fit prototypical spike templates consisting of a fast exponential rise and a slower exponential decay to each segment of data in a recording. The amplitude of the fit of each template is divided by the standard error of the fit to determine a criterion score at each possible data segment. A spike is detected when the criterion score exceeds a threshold, and the template with the highest criterion score for the spike is selected as a first-pass representation of the spike. We also described an extension of the matched-filter algorithm that finds the least-squares fit of data segments to the sum of two templates as a better representation of spikes that contain double-exponential decays. The superior performance of the detection algorithms compared to existing approaches was demonstrated using receiver operating characteristic plots.

The next step is estimation of spike parameters such as peak amplitude $I_{\max }$, and charge $\mathrm{Q}$ (Jankowski et al., 1993; Schroeder et al., 1992). $\mathrm{I}_{\max }$ indicates the maximal transmitter eflux rate and Q indicates the total amount of transmitter released. The kinetics of transmitter release have been described using the slope of a line fitted to two arbitrary points on the rising phase as a measure of release speed and the full-width-half-maximal time $\left(t_{50}\right)$ as a measure of release duration. Whereas these two parameters provide basic information about release kinetics, they do not fully describe the spike time course, which is often multi-exponential (Wang et al., 2001a). Fitting a function consisting of an exponential rise and a sum of one or two exponential decays can, in principle, fully describe the kinetics of typical spikes, but fitting exponential time constants is complicated in that the time course is a non-linear function of the time constants, therefore an iterative fitting routine must be applied. Iterative curve fitting is highly sensitive to seed values (initial parameter estimates), therefore a priori estimates of parameters are required to produce reliable fits to spikes. Here we describe an approach 
to use our previously described template-based match-filtering algorithm to provide seed values for the start time, amplitude and time constants to enable reliable curve fitting to spikes that vary considerably in time course.

Estimating parameters from spikes that overlap in time is problematic because they do not decay back to baseline and it is ambiguous to locate their start and end points. Previous algorithms sometimes fail to identify overlapping spikes, either incorrectly identifying two closely occuring spikes as a single spike and consequent skew estimates of spike parameters, or the mis-identifying the first of two overlapping spikes as a foot signal. Here we describe a user-independent automated overlap-identification algorithm that uses information about the time constant of decay from our matched-filter detection algorithm to reject spikes that are spaced too close in time to allow reliable parameter estimation.

Pre-spike foot signals provide information about the amount of neurotransmitter released through a narrow fusion pore prior to expansion and also the lifetime of the fusion pore. Determining the pre-spike baseline is critical for accurately identifying the starting time

of the pre-spike foot. Here we present a novel extension of our template-based detection algorithm that looks backwards from identified spikes to identify the flat baseline immediately preceding the pre-spike feature. The following sections describe the results of the estimation algorithm

\subsection{A two-criterion approach based on template fits successfully determines if successive spikes are overlapping}

Detecting and rejecting overlapping spikes is a necessary first step to make accurate estimates of spike parameters. Our recently described template-based detection algorithm 

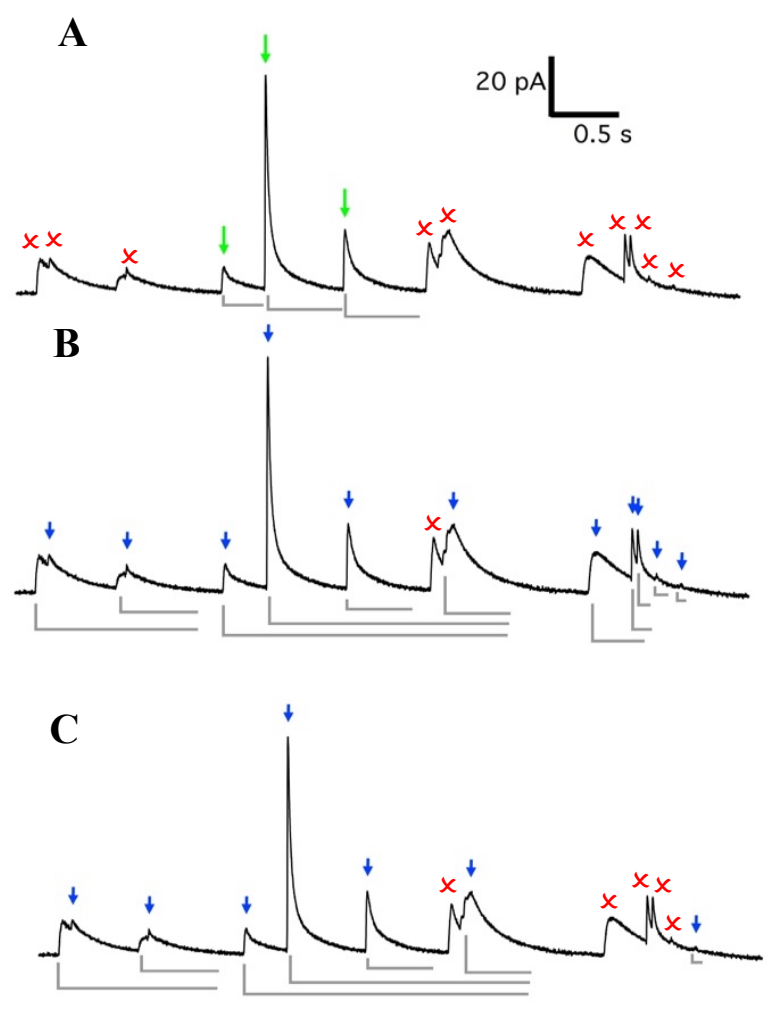

Fig. 4.1. A two-criteria approach successfully rejects overlapping spikes for parameter analyses

(A) Amperometric trace with many overlapping spikes. Green arrows indicate the three sufficiently separated spikes according to the dual-criteria algorithm whereas the red Xs indicate detected spikes that failed the test. The gray bars indicate the spike intervals determined by the algorithm. The end time of the spike is taken as three times the slower time constant determined following iterative fits to equation 12. (B) The same segment of amperometric trace with blue arrows indicating spikes accepted using a commonly used algorithm with an overlap threshold of $50 \%$. Note from the gray bars that the spike end times are misidentified by the algorithm. (C) Same as (B) using an overlap threshold of $0.5 \%$. generates estimates of

the decay time

constant(s) based on the

templates that produce

the highest criterion

score. In order to detect

overlapping spikes, the

decay time constant (or

slower time constant in

the case of spikes that

decay with two

exponentials), together

with the peak

amplitude $I_{\max }$, are used

to set the time window

within which a second

spike will be rejected

(Criterion 1 defined in

section 3.2.1). A second

spike is rejected if it

occurs within this time window because the first spike has not decayed sufficiently to

avoid disrupting the baseline of the second spike. 4.1(A) demonstrates that application of Criterion 1 allows retention of well separated for analysis whereas overlapping spikes are rejected. 4.1(B), (C) show that the a commonly used overlap rejection algorithm (Mosharov and Sulzer, 2005) fails to reliably reject overlapping spikes. In the Mosharov algorithm, the user sets the extent of allowable overlap, defined as the percentage ratio of 


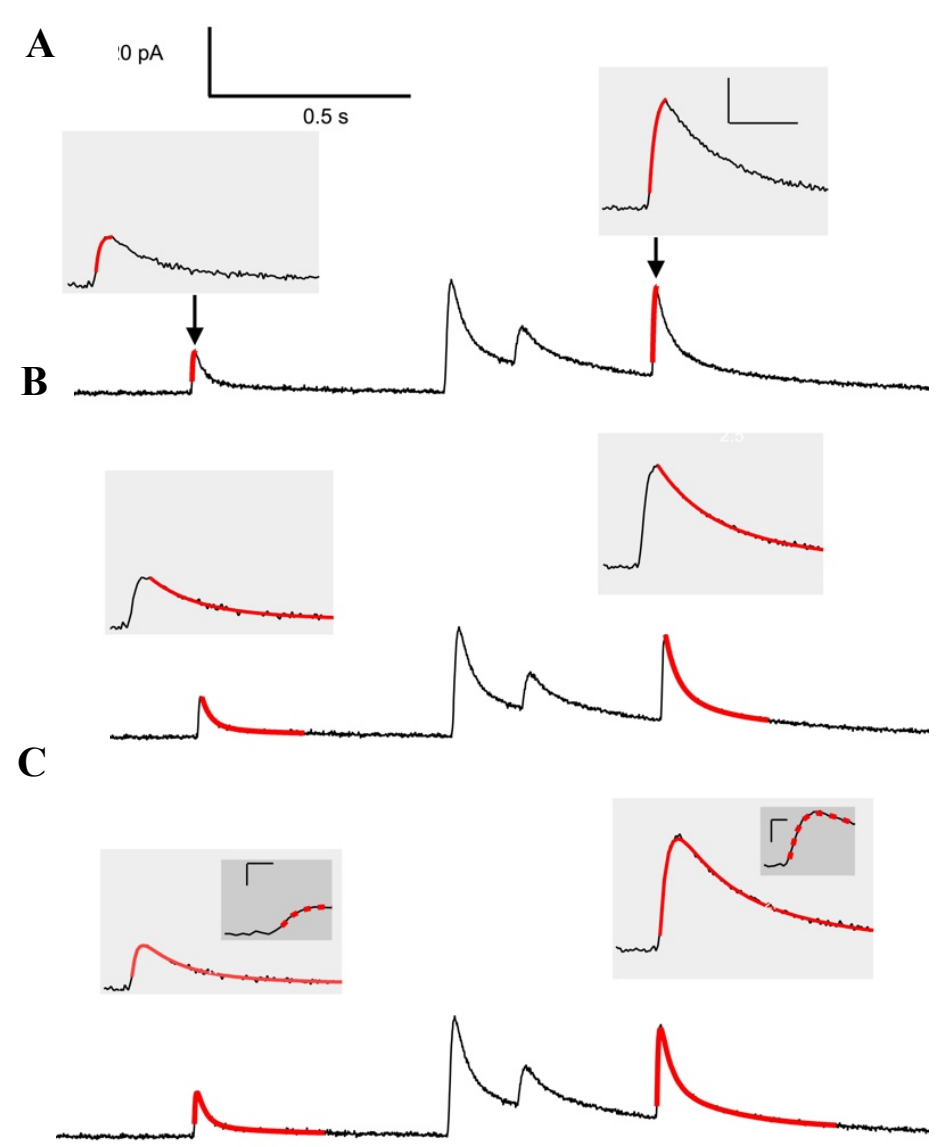

Fig. 4.2. A sequential fitting process results in excellent fits of equation 12 to well-separated spikes

(A) Red traces are fits to the rising phases of well-separated spikes (indicated by arrows). (B) Next, fits are made to the falling phase of spikes (red traces). (C) Finally, fits of equation 12 to the entire time course are made using seed parameters resulting from the fits to the rising and decaying phases. Insets demonstrate fits on an expanded time scale. Scale bars in light gray insets are 20 $\mathrm{pA}$ and $60 \mathrm{~ms}$ and scale bars are $10 \mathrm{pA}$ and $5 \mathrm{~ms}$ for dark gray the minima between

adjacent spikes and the

maximal height of the

taller spike. Spikes

accepted using an overlap

setting of $50 \%$ (a typical

level, (Mosharov and

Sulzer, 2005)) are shown

in Fig. 20(B), whereas

4.1(C) show that clearly

overlapping spikes are

accepted even for an

overlap setting as low as

$0.5 \%$. The inability to

reject overlaps

primarily results from

misidentification of the

ending time of a spike

(4.1(B), (C) gray bars)

whereas our algorithm, following iterative fitting, correctly identifies the end time of spikes (4.1(A)). An alternative approach to separate overlapping spikes described by Friedrich and Ashery (Friedrich and Ashery, 2010) is to smooth the data and then apply thresholds on minima to maxima ratios. Such an approach requires significant userintervention and filtering of data whereas our approach does not require filtering nor user-intervention. 


\subsection{Matched filtering template parameters can reliably seed an iterative curve fit of the entire spike time course by equation 12.}

Curve-fitting is an desirable means to extract spike parameters because it smooths random fluctations found in the raw data. Current approaches typically fit a line to an arbitrary segment of the rising phase (e.g., 25\% - 75\% $\mathrm{I}_{\max }$,(Mosharov and Sulzer, 2005)) as a measure of the rate that a fusion pore dilates to allow rapid release. In contrast, our algorithm fits an exponentially rising function to the rising phase of the spike, which provides a good fit to many spikes with less sensitivity to the selection of start and end points of a linear fit. On the other hand, iterative curve fitting is highly sensitive to the accuracy of initial seed values, however, the template fits that are obtained during matched filtering detection can aid in seeding an iterative fitting process. Our approach first fits the rising and decaying phases separately to improve the quality of seed values provided to the combined spike fit function, which contains up to seven free parameters (equation 12). 4.2(A), (B) and (C) show the fitting of the rising phase, decaying phase and entire spike, respectively, which produces excellent fits to typical spikes determined by visual inspection.

\subsection{Iterative curve fits provide accurate parameters for computing $Q$, Imax and t50}

Iterative curve fitting provides values for each spike $\left(\tau_{r}, \tau_{d 1,2,} a_{1,2}\right)$ for each spike which are used to compute traditional measures of spike amplitude and time course such as Q (charge), $\mathrm{t}_{50}$ (half-maximal width) and $\mathrm{I}_{\max }$ (peak amplitude). Data from bovine( Fig. 4.3) and mouse chromaffin cells (Fig. 4.4) were analyzed using both the new matched filter algorithm and that of Mosharov and Seltzer (Mosharov and Sulzer, 2005) to allow comparison of spike parameters. These were then 

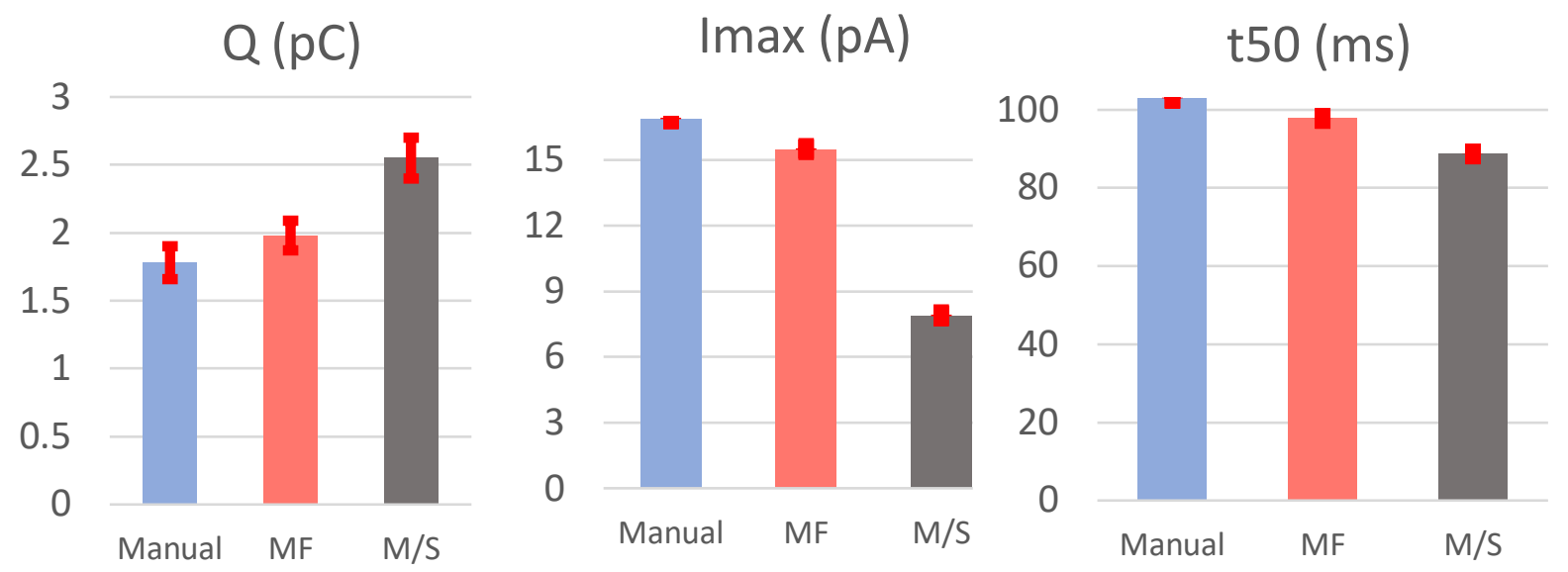

Fig. 4.4. Means of (a) $Q$, (b) $I_{\max }$ and (c) t50 calculated using MF, Manual and M/S (mosharov and sulzer, 2005) methods for amperometric recordings from bovine chromaffin cells
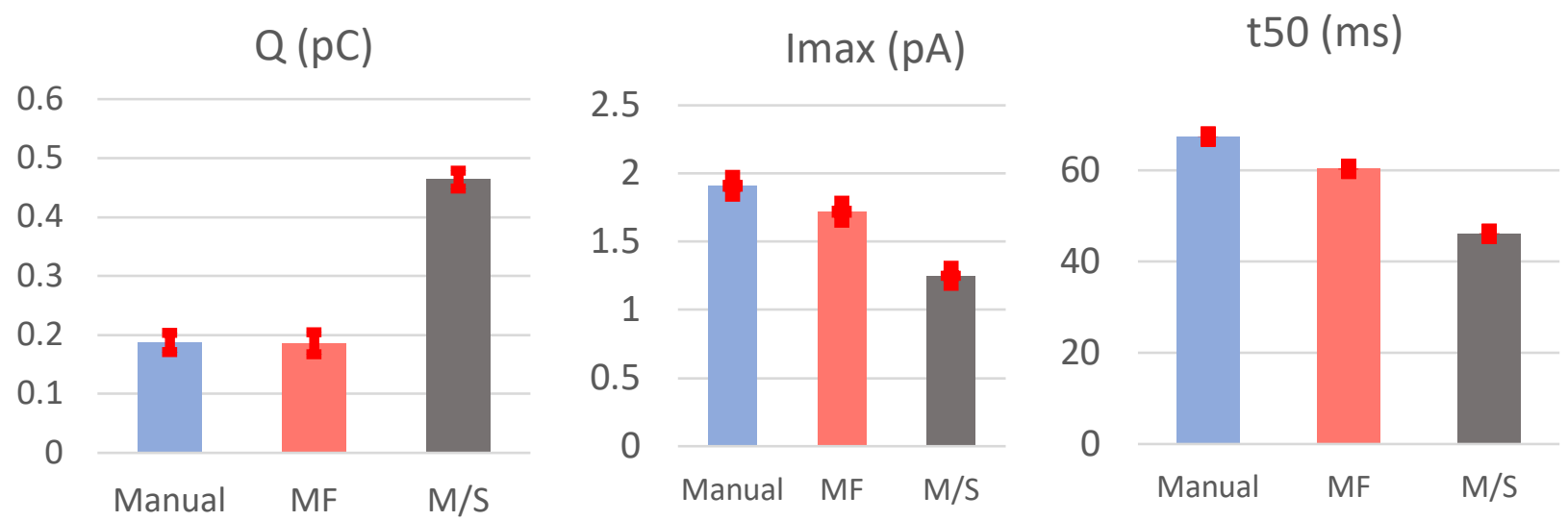

Fig. 4.3. Means of (a) $Q$, (b) $I_{\max }$ and (c) $t_{50}$ calculated using MF, Manual and M/S (mosharov and sulzer, 2005) methods for amperometric recordings from mouse chromaffin cells

compared with manual parameter estimates performed blindly with regard to the results of the other analyses. Median values obtained from the fully automated MF algorithm are generally within $\sim 10 \%$ of values obtained through laborious manual analyses (Table 4.1.) for both bovine and mouse chromaffin cell recordings, whereas the $\mathrm{M} / \mathrm{S}$ algorithm generally give poorer results. 4.5 presents histograms of the values for bovine (left column) and mouse (right column) chromaffin cells. It is typical to present histograms of Q raised to the one third 
Table 4.1. Medians of $\mathbf{Q}, \mathbf{I}_{\max }$, and $\mathbf{t}_{\mathbf{5 0}}$ computed for bovine and mouse chromaffin cell recordings using the new matched-filter based algorithm (MF), manual analysis, and the algorithm from (Mosharov and Sulzer, 2005) (M/S).

\begin{tabular}{|c|c|c|c|c|c|c|}
\hline & \multicolumn{6}{|c|}{ Medians } \\
\hline \multirow{3}{*}{$\begin{array}{l}\text { Statistic } \\
\text { Method }\end{array}$} & \multicolumn{3}{|c|}{ Bovine } & \multicolumn{3}{|c|}{ Mice } \\
\hline & MF & Manual & $\mathbf{M} / \mathbf{S}$ & MF & Manual & $\mathbf{M} / \mathbf{S}$ \\
\hline & $(\mathrm{n}=358)$ & $(\mathrm{n}=352)$ & $(\mathrm{n}=444)$ & $(\mathrm{n}=212)$ & $(\mathrm{n}=219)$ & $(\mathrm{n}=294)$ \\
\hline$Q(p C)$ & 1.27 & 1.21 & 1.40 & 0.0891 & 0.110 & 0.0742 \\
\hline$I_{\max }(\mathbf{p A})$ & 14.8 & 14.6 & 8.95 & 1.59 & 1.74 & 1.23 \\
\hline$t_{50}(\mathrm{~ms})$ & 108 & 100 & 97.7 & 60.0 & 64.1 & 42.1 \\
\hline
\end{tabular}
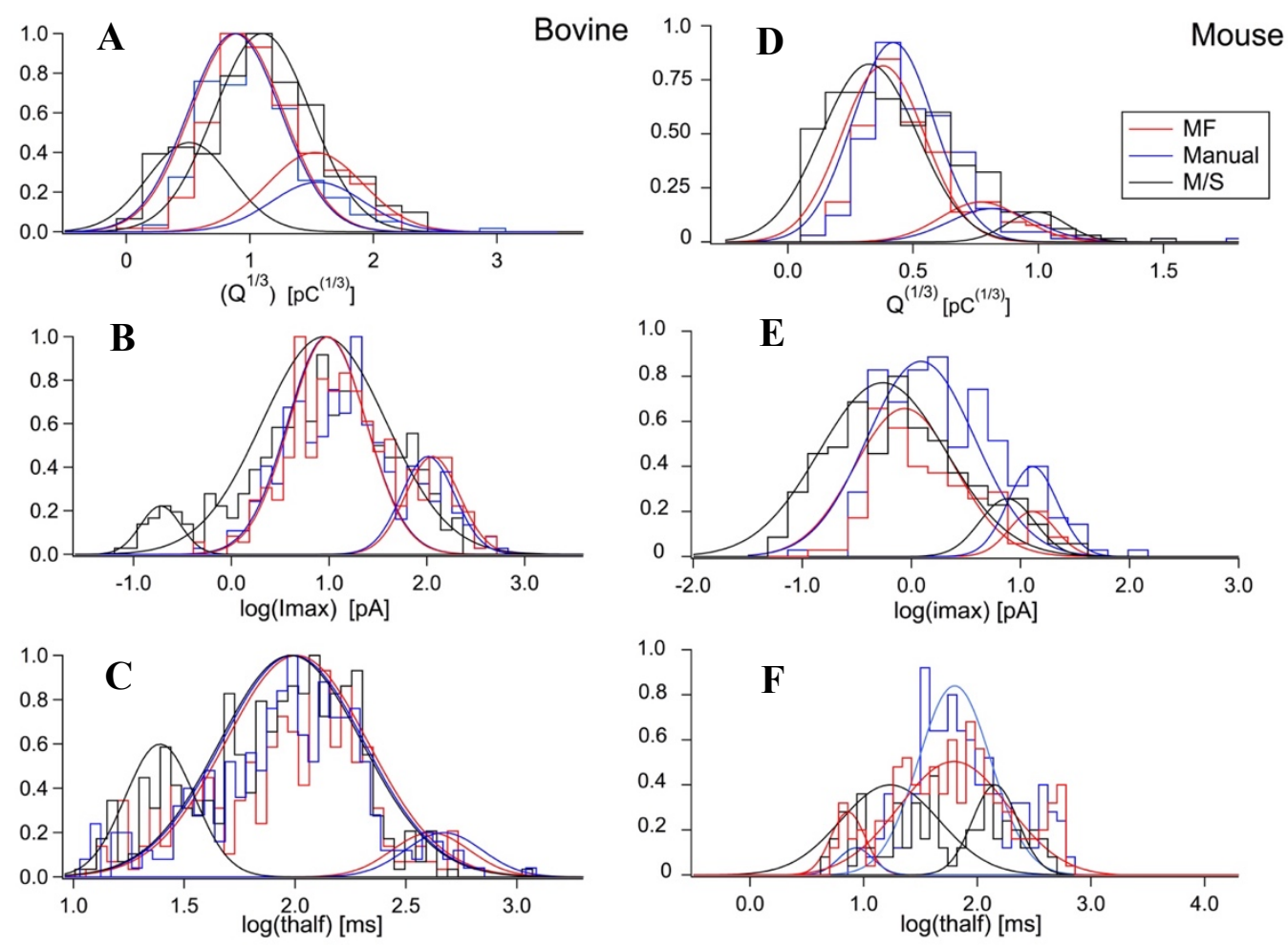

Fig. 4.5. Histograms of spike parameters indicate the excellent performance of the new MF parameter-estimation algorithm

Amperometric data from five bovine chromaffin cells (352 spikes, left column) and five mouse chromaffin cells (219 spikes, right column) were analyzed. In each histogram data from manual analysis are blue, the new MF algorithm is in red, and the M/S algorithm is black. Curves represent double Gaussian fits to the data. (A), (D) Histograms of $\mathrm{Q}^{1 / 3}$. (B), (E) Histograms of log transformed $I_{\max }$ values. (C), (F) Histograms of log transformed $t_{50}$ values. 
power (4.5(A), (D)) because charge is proportional to vesicle volume and vesicle radius typically has a Gaussian distribution or consists of two Gaussians representing two populations of vesicles (Jankowski et al., 1993; Schroeder et al., 1996). Log transformed parameters $t_{50}$ and $\mathrm{I}_{\max }$ also exhibit double Gaussian distributions (van Kempen et al., 2011). Gaussian fits for the fully automated MF algorithm are in good agreement with those obtained using manual analysis. Table 4.2. presents the Gaussian fit centers $\mu_{1,2}$ and spreads $\sigma_{1,2}$ for $\mathrm{Q}, \mathrm{I}_{\max }$ and $\mathrm{t}_{50}$ demonstrating good agreement between the MF algorithm and manual fits.

Table 4.2. Comparison of centers $(\mu 1,2)$ and spreads $(\sigma 1,2)$ of bi-modal gaussian distributions

\begin{tabular}{|c|c|c|c|c|c|c|c|c|c|c|c|c|}
\hline \multirow{3}{*}{$\begin{array}{l}\text { Statistic } \\
\text { Method }\end{array}$} & \multicolumn{12}{|c|}{ Bi-modal Gaussian fit parameters } \\
\hline & \multicolumn{6}{|c|}{ Bovine chromaffin cells } & \multicolumn{6}{|c|}{ Mouse chromaffin cells } \\
\hline & MF & Manual & $\mathrm{M} / \mathrm{S}$ & MF & Manual & $\mathrm{M} / \mathrm{S}$ & MF & Manual & $\mathrm{M} / \mathrm{S}$ & MF & Manual & $\mathrm{M} / \mathrm{S}$ \\
\hline$Q(p C)$ & 0.718 & 0.674 & 0.132 & 0.148 & 0.149 & 0.111 & 51.6 & 74.4 & 32.4 & 12.9 & 12.2 & 23.6 \\
\hline$I_{\max }(\mathbf{p A})$ & 9.47 & 9.65 & 0.189 & 3.65 & 3.71 & 1.95 & 0.866 & 1.22 & 0.545 & 4.47 & 4.98 & 6.62 \\
\hline \multirow[t]{2}{*}{$\mathbf{t}_{50}(\mathrm{~ms})$} & 468 & 408 & 95.5 & 1.76 & 1.76 & 2.86 & 62.1 & 62.8 & 143 & 4.79 & 4.21 & 1.92 \\
\hline & \multicolumn{3}{|c|}{$\mu_{2}$} & \multicolumn{3}{|c|}{$\sigma_{2}$} & \multicolumn{3}{|c|}{$\mu_{2}$} & \multicolumn{3}{|c|}{$\sigma_{2}$} \\
\hline Method & MF & Manual & $\mathrm{M} / \mathrm{S}$ & $\mathrm{MF}$ & Manual & $\mathrm{M} / \mathrm{S}$ & $\mathrm{MF}$ & Manual & $\mathrm{M} / \mathrm{S}$ & $\mathrm{MF}$ & Manual & $\mathrm{M} / \mathrm{S}$ \\
\hline $\mathrm{Q}(\mathbf{p C})$ & 3.51 & 3.52 & 1.33 & 0.161 & 0.166 & 0.166 & 457 & 542 & 950 & 12.1 & 14.3 & 2.23 \\
\hline $\mathbf{I}_{\max }(\mathbf{p A})$ & 114 & 103 & 8.91 & 2.45 & 2.43 & 84.5 & 12.8 & 12.8 & 7.54 & 1.99 & 2.09 & 2.25 \\
\hline $\mathbf{t}_{50}(\mathrm{~ms})$ & 94.3 & 97.7 & 24.7 & 3.92 & 2.86 & 1.62 & 7.26 & 8.51 & 18.1 & 1.63 & 1.63 & 3.25 \\
\hline
\end{tabular}




\subsection{Use of the criterion score from matched filtering can accurately identify pre-spike baseline for foot-signal analysis}

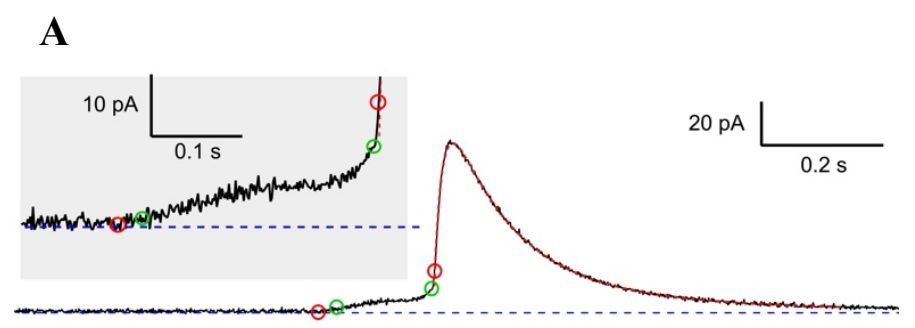

In the matched-filtering

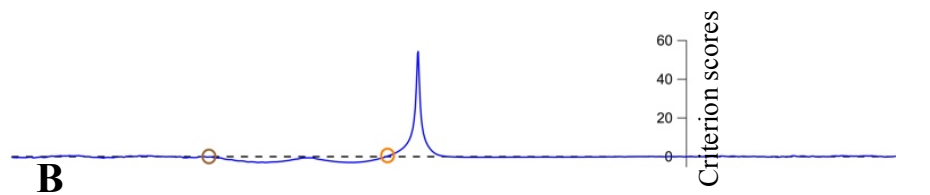

based foot detection

approach, the positive

criterion zero crossing

was used to determine

the baseline. Then the

first point that exceeded

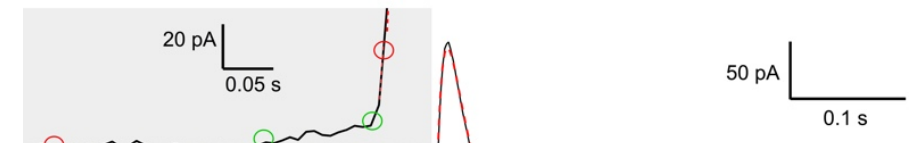

two standard deviations

above the baseline was

identified as the start of

the foot signal and the

end of the foot signal is

determined from $t_{0}$ of

the iterative curve fit to

the spike. Fig. 4.6

presents several types of

foot signals with the

green circles denoting

Fig. 4.6. The matched-filter based algorithm can accurately measure pre-spike foot signals with varying time courses

I he start and end times determined trom the MF algorithm are indicated as green circles and the red circles indicate start and end times for the $\mathrm{M} / \mathrm{S}$ algorithm. (A) Plateau-type foot signal (B) Ramp type foot signal (C) Sigmoidal foot signal. The blue traces are criterion scores. The orange circles denote positive zero crossing of the criterion score. The brown circles denote the beginning of the stable region of the criterion score used to determine the baseline. 
Table 4.3. presents the median values of foot charge and duration computed by the MF, manual and Mosharov and Sulzer approaches for amperometric recordings from five bovine chromaffin cells. Fig. 4.7 shows the mean values for foot parameters. The fully automated MF-based approach gives better agreement with manual measurements of the foot signal charge and duration than the M/S approach.

\subsection{Discussion}

The template-based matched filtering detection algorithm we recently described accurately detects spikes with few false positives (Balaji Ramachandran and Gillis,

2018). Here we show that the approach also generates initial parameter estimates useful

Qfoot $[\mathrm{pC}]$

120

100

80

60

40

20

0

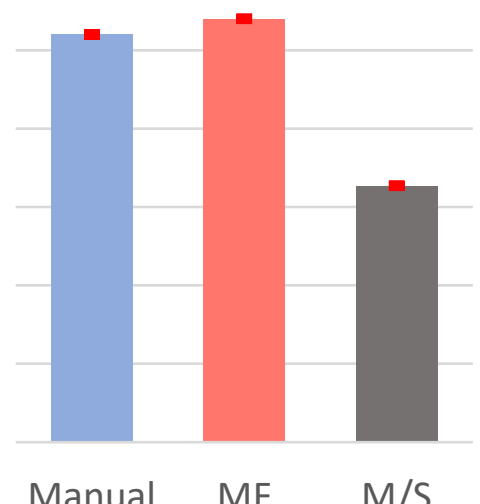

tfoot $[\mathrm{ms}]$

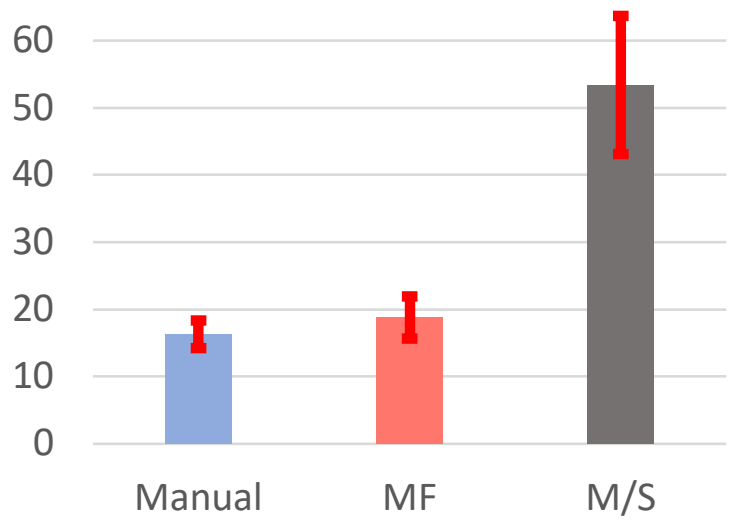

Fig. 4.7. Foot parameters of charge $\left(Q_{\text {foot }}\right)$ and duration $\left(t_{\text {foot }}\right)$ are shown as calculated by manual, $\mathrm{mf}$ and $\mathrm{m} / \mathrm{s}$ methods

information about the spike time course.

to detect overlapping spikes and seed iterative curve fitting to extract complete 
Table 4.3. Medians of foot parameters $t_{\text {foot }}$ and $Q_{\text {foot }}$ computed for bovine chromaffin cell recordings

\begin{tabular}{|l|lll|}
\hline & \multicolumn{3}{|c|}{ Bovine chromaffin cells } \\
\hline Statistic & \multicolumn{3}{|c|}{ Median } \\
Method & MF & Manual & M/S \\
& $(\mathrm{n}=128)$ & $(\mathrm{n}=105)$ & $(\mathrm{n}=333)$ \\
& 53.0 & 62.9 & 84.5 \\
Qfoot $_{\text {fC }}$ & 53.9 & 9.00 & 14.2 \\
$\mathrm{t}_{\text {foot }}(\mathrm{ms})$ & 6.50 & & \\
\hline
\end{tabular}

Overlapping spikes were reliably detected and eliminated from analyses using a dualcriteria approach. Note that this approach applies separate criteria for the first and second spike in a pair where the principle concern of accepting the first spike is that it has sufficient time to decay so that accurate decay time constants can be fit to the data. On the other hand, a more stringent criterion is applied to accept the second spike in a pair because the first spike must nearly completely decay to avoid disrupting the baseline for the second spike and allow foot analysis to proceed. Also note that the detection phase of analysis identifies essentially all spikes to allow accurate estimation of the frequency of exocytosis events near the electrode (Balaji Ramachandran and Gillis, 2018). However, only a subset of spikes can proceed to the analysis phase when events are detected at a high frequency because of overlap. This potentially introduces a bias in spike analysis if spike parameters are different at high release rates.

Our approach fits equation 12 to spikes to describe the complete amplitude and time course (not including the pre-spike foot) with either three (single exponential) or five (double exponential) parameters. This parameterization is a more complete description of typical spikes that parameters such as $\mathrm{I}_{\max }, \mathrm{Q}$, and $\mathrm{t}_{50}$. For example, two spikes may have the same $t_{50}$ but substantially different double-exponential decays. Thus, the parametrization by equation 12 can support different post-analyses of spike time course according to the wishes of the user. 
We find that equation 12 provides excellent fits to typical spikes in bovine and mouse chromaffin cells, but some events, e.g., "stand-alone feet" [ (Wang et al., 2003)] are not adequately described by equation 12 . In principle, templates can be created for other event waveforms and different fit functions can be devised to allow analysis of these events to proceed in a similar fashion to what is described in this work.

Whereas template fits during the detection phase provide reasonable seed values to allow iterative fitting of equation 12 to the data, we found that first fitting the rising and falling phases separately produces better results by allowing refinement of seed parameters before fitting equation 12 to the data. Visually good fits were obtained and the finely resolved parameter values are used to compute Q, t50 and Imax. Parameter histograms have centers and spreads within $12 \%$ and $4 \%$ of manually computed parameter histograms with an overall performance significantly better than the $\mathrm{M} / \mathrm{S}$ algorithms.

Finally, we show that the matched filtering criterion score-based baseline detection algorithm resulted in mean and median values of foot signal charge and duration within $3 \%$ and $11 \%$ of manually computed values. Developing algorithms to reliably determine baseline values within a fraction of a Pico-amp is more difficult than it might appear from visual examination of records. Use of the criterion score for this is novel, and has the advantage that scores are unit-less, so the method does not require prior knowledge of the noise standard deviation to find a stable region of the signal.

\subsection{Conclusions}

Our matched filtering-based spike and foot estimation algorithm effectively determines spike parameters in a fully automated manner and outperforms previous approaches. 


\section{Chapter 5}

\section{CONCLUSIONS AND FUTURE DIRECTIONS}

\subsection{Conclusions}

The goal of this study was to develop a technique to automatically detect and estimate spike and pre-spike signals in amperometric recordings. Through such automated analysis, amperometric events can be completely described in a few critical parameters. Since amperometric spikes are diverse in amplitudes and time courses, it is important that a detection algorithm accounts for this diversity. Existing algorithms either apply a derivative-threshold (Borges et al., 2008; Mosharov and Sulzer, 2005), template search approach (ClampFit) or amplitude threshold (Friedrich and Ashery, 2010) to detect spikes. Such approaches have disadvantages including the need for user-intervened data smoothing, bias against smaller/slower spikes and spurious detection. This dissertation showed that a template-library based "matched filter"(Balaji Ramachandran and Gillis, 2018) algorithm was efficient in amperometric spike detection, detecting $>97 \%$ of true spikes for a minimal false positive rate of $2 \%$. In order to estimate spikes, existing algorithms incorrectly estimate two overlapping spikes as one, rely on arbitrarily determined line-fits to the rising phase and auto-guessed decay phase fits leading to inaccurate parameter estimates. We demonstrated that parameters extracted from the MF detection were accurate enough to seed a non-linear curve fitting spike function that resulted in "good fits" to detected spikes. The good fits were then used to calculate critical spike parameters of $Q, t_{50}$ and $I_{\max }$. Histograms of these parameters compared favorably against manually computer parameter 
histograms. The next step was pre-spike foot signal analysis. Accurate pre-spike baseline estimation is important for foot signal analysis. While existing algorithms relied on steady-state searches on filtered amperometric traces to determine baseline, the MF algorithm used the criterion score to estimate prespike baseline. Once the baseline was determined, an amplitude threshold was applied in order to locate pre-spike foot signal start times and MF non-linear curve fits to spikes were used to set the foot signal end times.

\subsection{Spike detection}

Existing spike detection approaches such as derivative threshold (Borges et al., 2008; Gómez et al., 2002; Mosharov and Sulzer, 2005; Segura et al., 2000) rely heavily on low pass filtering of the amperometric recordings. As discussed in

Chapter 4, filtering has disadvantages including loss of small signals in smoothing or increase in false positives due to inadequate smoothing. Such a tradeoff situation demands intricate filter setting decisions to be made by the user of the algorithm. The MF detection approach does not require any low pass filtering since it relies on locating regions of maximum signal to noise ratio (criterion score) rather than mere signal maxima. Derivative threshold approach lays emphasis on the fastest rising region of the spike, tending to ignore the equally important decaying phase, leading to a bias against smaller and slower spikes. In contrast, the MF approach uses spike template functions that emphasize the decaying phase of the spike as much as the rising phase, in order to detect spikes. Manual curve fitting of all true spikes with an exponential rise and decay function was used to design the template library, consisting of 4 templates, that was used in 
the MF detection approach. The application of two thresholds, a higher threshold to capture criterion score maxima and a lower threshold to commence a next search for maxima ensured the rejection of spurious small signals. This is in contrast with existing template based approaches like ClampFit (ClampFit) which merely average user-identified spikes to form templates and apply a single threshold on a detection criterion to identify spikes. Detection applications using baseline subtraction and amplitude thresholds (Friedrich and Ashery, 2010) fail due to the wavering nature of amperometric baseline and also detect spurious small-amplitude flickers as spikes. Plotting receiver operating characteristics was a rigorous method to measure the sensitivity vs specificity of detection algorithms. At criterion score thresholds between 2 and 3,>95\% of spikes were detected for $<5 \%$ false positives, by the MF algorithm which also outperformed existing commercial and freeware applications.

\subsection{Estimation of spike parameters}

In order to estimate amperometric spikes, we first identified and reject overlapping spikes. Derivative threshold (Mosharov and Sulzer, 2005) based algorithm relies on the user to decide the extent of overlapping spikes to be allowed. Such user-settings are extensive and not straightforward, often leading to incorrect identification of two overlapping spikes as one, or the identification of the first of two overlapping spikes as a foot-signal. This dissertation demonstrated a two-condition based overlap rejection algorithm that rejected the second of adjacent spikes it occurred prior to the decay of the first spike to a minimum amplitude and then rejected the first spike if it did not decay to 3 times the decay 
time constant extracted from the MF-template parameters. Such an approach ensured that overlapping spikes could be rejected without user-intervention. Traditional DT based algorithm performed the estimation of spikes by line fitting to arbitrary segments of rising phases of spikes and auto-guessed non-linear curve fitting to the decaying phase.

This dissertation showed that an exponential-rise and exponential decay template could accurately fit spikes based on initial seed values provided by MF template parameters. By employing a two-step fitting approach of first fitting the rising and decaying phases of the spike with separate non-linear exponential functions, and then fitting the complete spike function based on seed values from the first step, the MF estimation approach ensured that output spike parameters improved in accuracy with each fitting step. Using non-linear curve fit spike information like rise time constant $\left(\tau_{\mathrm{r}}\right)$, decay time constants $\left(\tau_{\mathrm{d} 1,2}\right)$, amplitudes $\left(\mathrm{a}_{1,2}\right)$, baseline $(\mathrm{b})$, key spike parameters of $t_{50}, I_{\max }$ and Q were calculated. Histograms of parameters showed bi-modal Gaussian populations that compare more favorably against manual computations than existing derivative threshold-based estimation algorithms (Mosharov and Sulzer, 2005).

\subsection{Detection and estimation of pre-spike foot signals}

This study showed that accurate detection of spike start times and baseline current at the MF criterion score peaks and zeros respectively, enabled detection of foot signals. The beginning points of pre-spike foot signals were detected by walking backwards from spike start times to locate the criterion score "zero" or the baseline current. The end points of the foot signals were located at the intersection 
of the baseline with the rising phase of extrapolated fits to the spikes. The foot duration $t_{\text {foot }}$ and charge $\mathrm{Q}_{\text {foot }}$ were then calculated from the second order polynomial fits to the foot regions. The foot duration corresponds to the lifetime of the narrow fusion pore before it expands and the foot charge corresponds to the small amount of neurotransmitter leaking slowly out of the narrow pore(Wightman et al., 1991).Hence the detection and estimation of amperometric signals, namely spikes and foot signals, was implemented in this study.

\subsection{Future directions}

\subsubsection{Classifying pre-spike foot signals}

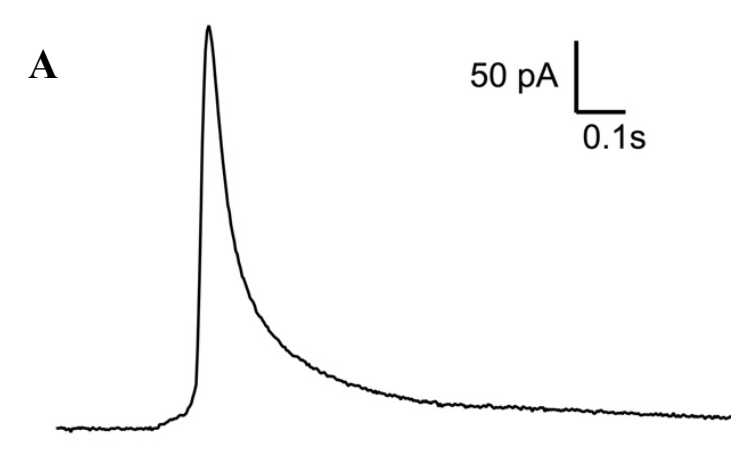

Amatore et al (Amatore et al., 2009)

classify foot signals into two types, based on their shapes, namely (a)

ramp and (b) ramp + plateau and

hypothesize that the fusion pore diameter controls the foot signal

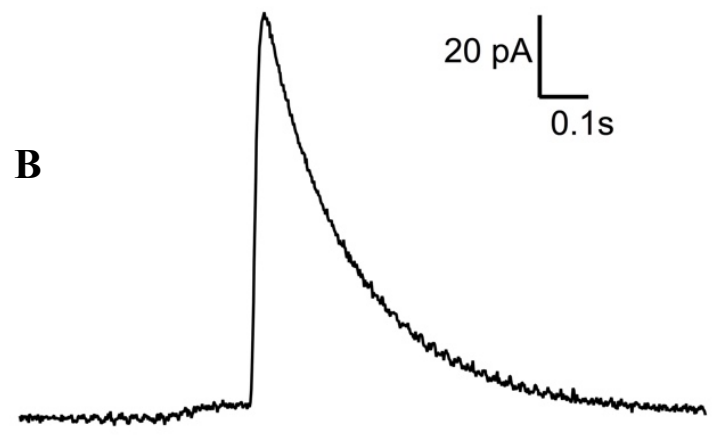

Fig. 5.1. Classifying foot signals

(A) Ramp type foot preceding a spike (B) Ramp + Plateau type foot preceding a spike found in amperometric recordings made from a bovine chromaffin cell shape. The MF based foot analysis

algorithm can detect both types of

foot signals irrespective of their

shapes. Automated classification of

foot signals will enable to understand

if the fusion pore dwelled at a narrow

diameter (plateau shape) prior to 
expansion. Such classification could be done by looking for the presence or absence of a steady state (plateau) in the amplitude of the foot signal region.

\subsubsection{Post-spike foot signals}

Mellander et al (Mellander et al., 2012) hypothesize that the occurrence of abrupt plateaus at the end of PC12 spikes is a type of regulation that occurs within a transmission event. Such phenomenon was described as "extended kiss-and-run"

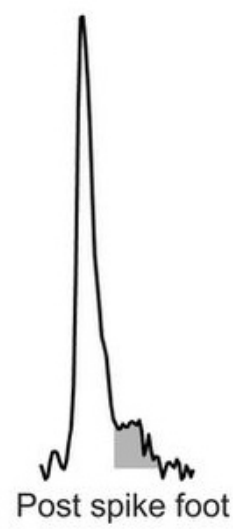

Fig. 5.2. Post-spike foot signal

Reproduced from Mellander et al (Mellander et al., 2012) with copyright permission from the Nature Publishing Group.

exocytosis observed as "post spike foot signals". The detection and analysis of post-spike foot signals will provide understanding about the temporal characteristics of exocytosis regulation, particularly when it occurs during transmission. Spikes decay to $99 \%$ of their peak value within 3 decay time constants. First a criterion score "zero", or baseline in the region of the trace beyond 3 decay time constants from a spike peak must be located. Next in the region between the peak of the spike and the criterion score zero, if a steady state amplitude plateau is found, a post-spike foot signal is said to be detected. The 
steady state can be found by comparing adjacent segments to assess if they are within one standard deviation of each other.

\subsubsection{Stand-alone foot signals}

Some groups report almost rectangular shaped stand-alone foot signals recorded by amperometry in PC12 cells ((van Kempen et al., 2011; Wang et al., 2003) and mice chromaffin cells(van Kempen et al., 2011). These signals are hypothesized to be due to fusion pores that reach a steady diameter and close without further

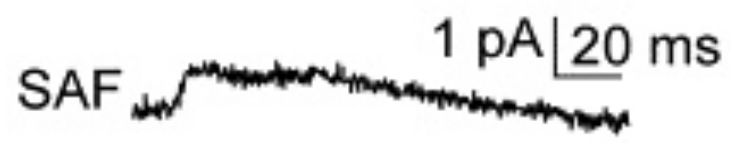

Fig. 5.3. Stand-alone-foot (SAF)

Signal recorded from a chromaffin cell. Reproduced from (van Kempen et al., 2011)

expansion. Since their rise time constants are similar to those of amperometric spikes, and amplitudes are small ( 2 pA) (van Kempen et al., 2011), an amplitude cut-off on identified spikes can be used to isolate potential stand-alone foot signals following which testing for rectangular shapes must be done. Other approaches could involve setting a lower and upper cutoff range on criterion scores and then testing detected events for SAF rectangular shape.

\subsubsection{Automated template library design}

MF templates were designed using information obtained from manual detection, curve fitting and estimation of spikes. Such manual analysis is time consuming. There is hence a requirement of automated spike template design. In order to design a template library, a few templates that represent amperometric spikes of 
an entire species should be identified. The MF algorithm detects "spikes" by looking for exceptional matches between user-designed templates and the amperometric recording. A "matched filter" clustering algorithm can be used to compare user-identified "spikes" against each other and group them into clusters based on how well they match each other. Although there are several distance metrics (Li et al., 2011) including Euclidean and Manhattan distances to determine similarity, in this case the MF criterion score can be used as the distance metric for clustering since the criterion score metric was successful in MF detection. The cluster means would serve as templates to be included in an automated template library. This method will provide a more appropriate distance metric and minimal user-involvement compared to traditional clustering algorithms such as K-means (Lloyd, 1982).

\subsubsection{DT -MF hybrid spike detection approach}

The major advantage of the DT algorithm (Mosharov and Sulzer, 2005) is that it is fast. The MF algorithm compares templates to data segments in a point-bypoint fashion that maybe computationally expensive in real-time analysis. By first applying a quick DT algorithm at a low threshold, to identify potential spike regions and then applying MF approach to only those regions, the speed of detection can be increased. Thus, a combined DT-MF approach is necessary for optimizing the advantages of speed in DT and accuracy in the MF approach.

In summary, the MF detection approach was efficient in spike detection and provided good initial guess for non-linear curve fitting and spike parameter estimation. The accuracy of MF spike detection helped in identifying foot signals 
in the pre-spike regions. The classification of pre-spike foot signals based on temporal dynamics, estimation of post-spike and stand-alone foot signals and the design of an automated template library are future directions. The future directions of this study should impact our understanding of amperometric signals and consequently of the physiological mechanism of fusion and cargo release. 


\section{REFERENCES}

Compound versus multigranular exocytosis in peritoneal mast cells. The Journal of General Physiology, 1990; 95: 397-409.

Temporal coincidence between synaptic vesicle fusion and quantal secretion of acetylcholine. The Journal of Cell Biology, 1985; 101: 1386-99.

Alberts B BD, Lewis J, et al. . Transport from the Trans Golgi Network to the Cell Surface: Exocytosis. Molecular Biology of the Cell. Garland Science; 1994.: New York:, 2002.

Albillos A, Dernick G, Horstmann H, Almers W, de Toledo GA, Lindau M. The exocytotic event in chromaffin cells revealed by patch amperometry. Nature, 1997; 389: 509.

Alvarez de Toledo G, Fernandez-Chacon R, Fernandez JM. Release of secretory products during transient vesicle fusion. Nature, 1993; 363: 554-8.

Amatore C, Arbault S, Bonifas I, Bouret Y, Erard M, Ewing AG, Sombers LA. Correlation between Vesicle Quantal Size and Fusion Pore Release in Chromaffin Cell Exocytosis. Biophysical Journal; 88: 4411-20.

Amatore C, Arbault S, Bonifas I, Guille M. Quantitative investigations of amperometric spike feet suggest different controlling factors of the fusion pore in exocytosis at chromaffin cells. Biophysical Chemistry, 2009; 143: 124-31.

Amatore C, Arbault S, Bonifas I, Guille M, Lemaître F, Verchier Y. Relationship between amperometric pre-spike feet and secretion granule composition in Chromaffin cells: An overview. Biophysical Chemistry, 2007; 129: 181-9.

Amatore C, Arbault S, Chen Y, Crozatier C, Lemaître F, Verchier Y. Coupling of Electrochemistry and Fluorescence Microscopy at Indium Tin Oxide Microelectrodes for the Analysis of Single Exocytotic Events. Angewandte Chemie International Edition, 2006; 45: 4000-3. 
Andrew FD, Gregor D, Vicente V, Ming GY, Conrad DJ, Harold GC, Manfred L. An electrochemical detector array to study cell biology on the nanoscale. Nanotechnology, 2002; 13: 285.

Aoki KR. Botulinum Toxin: A Successful Therapeutic Protein. Current Medicinal Chemistry, 2004; 11: 3085-92.

Avantor.

Balaji Ramachandran S, Gillis KD. A matched-filter algorithm to detect amperometric spikes resulting from quantal secretion. Journal of Neuroscience Methods, 2018; 293: 338-46.

Banks P, Helle K. The release of protein from the stimulated adrenal medulla. Biochemical Journal, 1965; 97: 40C-1C.

Barizuddin S, Liu X, Mathai JC, Hossain M, Gillis KD, Gangopadhyay S. Automated Targeting of Cells to Electrochemical Electrodes Using a Surface Chemistry Approach for the Measurement of Quantal Exocytosis. ACS Chemical Neuroscience, 2010; 1: 590-7.

Bell MR. IEEE Transactions on Information Theory, 1993a.

Bell MR. Information theory and radar waveform design. IEEE Transactions on Information Theory, 1993b; 39: 1578-97.

Biermann T, Bonsch D Fau - Reulbach U, Reulbach U Fau - Kornhuber J, Kornhuber J Fau - Bleich S, Bleich S. Dopamine and N-methyl-D-aspartate receptor expression in peripheral blood of patients undergoing alcohol withdrawal.

Birks R, Huxley HE, Katz B. The fine structure of the neuromuscular junction of the frog. The Journal of Physiology, 1960; 150: 134-44.6.

Bokvist K, Holmqvist M, Gromada J, Rorsman P. Compound exocytosis in voltage-clamped mouse pancreatic $\beta$-cells revealed by carbon fibre amperometry. Pflügers Archiv, 2000; 439: 634-45. 
Borges R, Camacho M, Gillis KD. Measuring secretion in chromaffin cells using electrophysiological and electrochemical methods. Acta Physiologica, 2008; 192: 173-84.

Borges R, Machado J D, Betancor G, Camacho M. Pharmacological Regulation of the Late Steps of Exocytosis. Annals of the New York Academy of Sciences, 2006; 971: 184-92.

Brisinda G, Bentivoglio AR, Maria G, Albanese A. Treatment with botulinum neurotoxin of gastrointestinal smooth muscles and sphincters spasms. Movement Disorders, 2004; 19: S146-S56.

CAD.

Ceccarelli B, Hurlbut WP, Mauro A. TURNOVER OF TRANSMITTER AND SYNAPTIC VESICLES AT THE FROG NEUROMUSCULAR JUNCTION. The Journal of Cell Biology, 1973; 57: 499.

Chang C-W, Chiang C-W, Jackson MB. Fusion pores and their control of neurotransmitter and hormone release. The Journal of General Physiology, 2017.

Chen P, Xu B, Tokranova N, Feng X, Castracane J, Gillis KD. Amperometric Detection of Quantal Catecholamine Secretion from Individual Cells on Micromachined Silicon Chips. Analytical Chemistry, 2003; 75: 518-24.

Chow RH, Ludolf Von Rüden. Electrochemical detection of secretion from single cells. Single-channel recording. Springer, New York,: pp 245-75.

Chow RH, von Ruden L, Neher E. Delay in vesicle fusion revealed by electrochemical monitoring of single secretory events in adrenal chromaffin cells. Nature, 1992a; 356: 60-3.

Chow RH, von Ruden L, Neher E. Delay in vesicle fusion revealed by electrochemical monitoring of single secretory events in adrenal chromaffin cells. Nature, 1992b; 356: 60-3.

ClampFit. ClampFit 10. Molecular devices. 
Clements JD, Bekkers JM. Detection of spontaneous synaptic events with an optimally scaled template. Biophysical Journal, 1997; 73: 220-9.

Colliver TL, Hess EJ, Pothos EN, Sulzer D, Ewing AG. Quantitative and Statistical Analysis of the Shape of Amperometric Spikes Recorded from Two Populations of Cells. Journal of Neurochemistry, 2000; 74: 1086-97.

Constable JRL, Graham ME, Morgan A, Burgoyne RD. Amisyn Regulates Exocytosis and Fusion Pore Stability by Both Syntaxin-dependent and Syntaxinindependent Mechanisms. Journal of Biological Chemistry, 2005; 280: 31615-23.

Davidsson P, Gottfries J, Bogdanovic N, Ekman R, Karlsson I, Gottfries C-G, Blennow K. The synaptic-vesicle-specific proteins rab3a and synaptophysin are reduced in thalamus and related cortical brain regions in schizophrenic brains. Schizophrenia Research; 40: 23-9.

Davis AF, Bai J, Fasshauer D, Wolowick MJ, Lewis JL, Chapman ER. Kinetics of Synaptotagmin Responses to $\mathrm{Ca} 2+$ and Assembly with the Core SNARE Complex onto Membranes. Neuron, 1999; 24: 363-76.

de Duve C. The Lysosome Concept. in Ciba Foundation Symposium Lysosomes (eds A. V. S. de Reuck and M. P. Cameron), J doi: 10.1002/9780470715314.ch1. John Wiley \& Sons, Ltd, . : Chichester, UK, 1963.

de Toledo GA, Fernandez-Chacon R, Fernandez JM. Release of secretory products during transient vesicle fusion. Nature, 1993a; 363: 554-8.

de Toledo GA, Fernández-Chacón R, Fernández JM. Release of secretory products during transient vesicle fusion. Nature, 1993b; 363: 554.

de Wit H, Walter AM, Milosevic I, Gulyás-Kovács A, Riedel D, Sørensen JB, Verhage M. Synaptotagmin-1 Docks Secretory Vesicles to Syntaxin-1/SNAP-25 Acceptor Complexes. Cell; 138: 935-46.

Doherty GJ, McMahon HT. Mechanisms of Endocytosis. Annual Review of Biochemistry, 2009; 78: 857-902. 
Elgrishi N, Rountree KJ, McCarthy BD, Rountree ES, Eisenhart TT, Dempsey JL. A Practical Beginner's Guide to Cyclic Voltammetry. Journal of Chemical Education, 2018; 95: 197-206.

Esquenazi A, Mayer N. Botulinum toxin for the management of muscle overactivity and spasticity after stroke.

Evanko D. Primer: spying on exocytosis with amperometry. Nat Meth, 2005; 2: $650-$.

Fatt, Katz. Spontaneous subthreshold activity at motor nerve endings. 1952.

Fatt P, Katz B. An analysis of the end-plate potential recorded with an intracellular electrode. The Journal of Physiology, 1951; 115: 320-70.

Fesce R, Grohovaz F, Valtorta F, Meldolesi J. Neurotransmitter release: fusion or 'kiss-and-run'? Trends in Cell Biology, 1994; 4: 1-4.

Finnegan JM, Wightman RM. Correlation of Real-time Catecholamine Release and Cytosolic $\mathrm{Ca} 2+$ at Single Bovine Chromaffin Cells. Journal of Biological Chemistry, 1995; 270: 5353-9.

Frampton JE, Easthope SE. Botulinum toxin A (Botox Cosmetic): a review of its use in the treatment of glabellar frown lines.

Friedrich R, Ashery U. From spike to graph - A complete automated single-spike analysis. Journal of Neuroscience Methods, 2010; 193: 271-80.

Gao Y, Bhattacharya S, Chen X, Barizuddin S, Gangopadhyay S, Gillis KD. A microfluidic cell trap device for automated measurement of quantal catecholamine release from cells. Lab on a Chip, 2009; 9: 3442-6.

Gao Y, Chen X, Gupta S, Gillis KD, Gangopadhyay S. Magnetron sputtered diamond-like carbon microelectrodes for on-chip measurement of quantal catecholamine release from cells. Biomedical Microdevices, 2008; 10: 623. 
Gillis KD, Liu XA, Marcantoni A, Carabelli V. Electrochemical measurement of quantal exocytosis using microchips. Pflügers Archiv - European Journal of Physiology, 2017.

Gómez JF, Brioso MA, Machado JD, SÁNchez JL, Borges R. New Approaches for Analysis of Amperometrical Recordings. Annals of the New York Academy of Sciences, 2002; 971: 647-54.

HarrickSci.

Hastoy B, Clark A, Rorsman P, Lang J. Fusion pore in exocytosis: More than an exit gate? A $\beta$-cell perspective. Cell Calcium, 2017; 68: 45-61.

Heuser JE, Reese TS. EVIDENCE FOR RECYCLING OF SYNAPTIC VESICLE MEMBRANE DURING TRANSMITTER RELEASE AT THE FROG NEUROMUSCULAR JUNCTION. The Journal of Cell Biology, 1973; 57: 31544.

Heuser JE, Reese TS, Dennis MJ, Jan Y, Jan L, Evans L. Synaptic vesicle exocytosis captured by quick freezing and correlated with quantal transmitter release. The Journal of Cell Biology, 1979; 81: 275.

Horvath E, Kovacs K. Misplaced exocytosis: Distinct ultrastructural feature in some pituitary adenomas, 1974.

Ichikawa A. FINE STRUCTURAL CHANGES IN RESPONSE TO HORMONAL STIMULATION OF THE PERFUSED CANINE PANCREAS. The Journal of Cell Biology, 1965; 24: 369-85.

Imig C, Min S-W, Krinner S, Arancillo M, Rosenmund C, Südhof Thomas C, Rhee J, Brose N, Cooper Benjamin H. The Morphological and Molecular Nature of Synaptic Vesicle Priming at Presynaptic Active Zones. Neuron; 84: 416-31.

Jahn R, Fasshauer D. Molecular machines governing exocytosis of synaptic vesicles. Nature, 2012; 490: 201-7.

Jankowski JA, Schroeder TJ, Ciolkowski EL, Wightman RM. Temporal characteristics of quantal secretion of catecholamines from adrenal medullary cells. Journal of Biological Chemistry, 1993; 268: 14694-700. 
Jewell JL, Oh E, Thurmond DC. Exocytosis mechanisms underlying insulin release and glucose uptake: conserved roles for Munc18c and syntaxin 4. American Journal of Physiology - Regulatory, Integrative and Comparative Physiology, 2010; 298: R517-R31.

Jewell ML, Breyer RM, Currie KPM. Regulation of Calcium Channels and Exocytosis in Mouse Adrenal Chromaffin Cells by Prostaglandin EP3 Receptors. Molecular Pharmacology, 2011; 79: 987-96.

Jung JH, Szule JA, Marshall RM, McMahan UJ. Variable priming of a docked synaptic vesicle. Proceedings of the National Academy of Sciences of the United States of America, 2016; 113: E1098-E107.

Kim S, McNames J. Automatic spike detection based on adaptive template matching for extracellular neural recordings. Journal of Neuroscience Methods, 2007; 165: 165-74.

Kiran R, Rousseau L, Lissorgues G, Scorsone E, Bongrain A, Yvert B, Picaud S, Mailley P, Bergonzo P. Multichannel Boron Doped Nanocrystalline Diamond Ultramicroelectrode Arrays: Design, Fabrication and Characterization. Sensors, $2012 ; 12$.

Kirshner N, Sage HJ, Smith WJ, Kirshner AG. Release of Catecholamines and Specific Protein from Adrenal Glands. Science, 1966; 154: 529-31.

Kisler K, Kim BN, Liu X, Berberian K, Fang Q, Mathai CJ, Gangopadhyay S, Gillis KD, Lindau M. Transparent Electrode Materials for Simultaneous Amperometric Detection of Exocytosis and Fluorescence Microscopy. Journal of biomaterials and nanobiotechnology, 2012; 3: 243-53.

Klyachko VA, Jackson MB. Capacitance steps and fusion pores of small and large-dense-core vesicles in nerve terminals. Nature, 2002; 418: 89.

Laurell.

Leszczyszyn DJ, Jankowski Ja Fau - Viveros OH, Viveros Oh Fau - Diliberto EJ, Jr., Diliberto Ej Jr Fau - Near JA, Near Ja Fau - Wightman RM, Wightman RM. Nicotinic receptor-mediated catecholamine secretion from individual chromaffin cells. Chemical evidence for exocytosis. 1990. 
Levenberg K. A method for the solution of certain non-linear problems in least squares. Quart. Appl. Math., 1944; V 2: 164--8

Li Z, Ding Q, Zhang W. A Comparative Study of Different Distances for Similarity Estimation. In Chen R, editor. Intelligent Computing and Information Science: International Conference, ICICIS 2011, Chongqing, China, January 8-9, 2011. Proceedings, Part I. Springer Berlin Heidelberg: Berlin, Heidelberg, 2011: 483-8.

Liu X, Barizuddin S, Shin W, Mathai CJ, Gangopadhyay S, Gillis KD. A microwell device for targeting single cells to electrochemical microelectrodes for high-throughput amperometric detection of quantal exocytosis. Analytical chemistry, 2011; 83: 2445-51.

Liu Y, Schirra C, Edelmann L, Matti U, Rhee J, Hof D, Bruns D, Brose N, Rieger $\mathrm{H}$, Stevens DR, Rettig J. Two distinct secretory vesicle-priming steps in adrenal chromaffin cells. The Journal of Cell Biology, 2010; 190: 1067-77.

Lloyd S. Least squares quantization in PCM. IEEE Transactions on Information Theory, 1982; 28: 129-37.

Mabbott GA. An introduction to cyclic voltammetry. Journal of Chemical Education, 1983; 60: 697.

Macpherson JV. A practical guide to using boron doped diamond in electrochemical research. Physical Chemistry Chemical Physics, 2015; 17: 293549.

Man KNM, Imig C, Walter AM, Pinheiro PS, Stevens DR, Rettig J, Sørensen JB, Cooper BH, Brose N, Wojcik SM. Identification of a Munc13-sensitive step in chromaffin cell large dense-core vesicle exocytosis. eLife, 2015; 4: e10635.

Marquardt D. An Algorithm for Least-Squares Estimation of Nonlinear Parameters. Journal of the Society for Industrial and Applied Mathematics, 1963; 11: 431-41.

Martin RS, Gawron AJ, Fogarty BA, Regan FB, Dempsey E, Lunte SM. Carbon paste-based electrochemical detectors for microchip capillary electrophoresis/electrochemistry. Analyst, 2001; 126: 277-80. 
Mellander LJ, Trouillon R, Svensson MI, Ewing AG. Amperometric post spike feet reveal most exocytosis is via extended kiss-and-run fusion. Scientific Reports, 2012; 2: 907.

Mesbah M, Khlif M, Boashash B, Colditz P. Newborn EEG seizure detection using optimized time-frequency matched filter. 2007 9th International Symposium on Signal Processing and Its Applications, 2007: 1-4.

Microchem.

Mohrmann R, de Wit H, Connell E, Pinheiro PS, Leese C, Bruns D, Davletov B, Verhage M, Sørensen JB. Synaptotagmin Interaction with SNAP-25 Governs Vesicle Docking, Priming, and Fusion Triggering. The Journal of Neuroscience, 2013; 33: 14417.

Mosharov EV, Sulzer D. Analysis of exocytotic events recorded by amperometry. Nat Meth, 2005; 2: 651-8.

Nakamura H, Nagai K, Yoshida M. Application of Independent Component Analysis and Expansion Matching Filter for Automatic Detection of Motor Unit Action Potential Trains. 2005 IEEE Engineering in Medicine and Biology 27th Annual Conference, 2005: 5013-6.

Oheim M, Loerke D, Stühmer W, Chow RH. The last few milliseconds in the life of a secretory granule. European Biophysics Journal, 1998; 27: 83-98.

Perrais D, Kleppe IC, Taraska JW, Almers W. Recapture after exocytosis causes differential retention of protein in granules of bovine chromaffin cells. The Journal of Physiology, 2004; 560: 413-28.

Pickett JA, Edwardson JM. Compound Exocytosis: Mechanisms and Functional Significance. Traffic, 2006; 7: 109-16.

Poor VH. An Introduction to Signal Detection and Estimation: Edition 2. Springer Science \& Business Media, 2013.

Rizo J, Chen X, Araç D. Unraveling the mechanisms of synaptotagmin and SNARE function in neurotransmitter release. Trends in Cell Biology; 16: 339-50. 
Rizo J, Rosenmund C. Synaptic vesicle fusion. Nature structural \& molecular biology, 2008; 15: 665-74.

Rohm H.

Rossier Joël S, Schwarz A, Reymond F, Ferrigno R, Bianchi F, Girault Hubert H. Microchannel networks for electrophoretic separations. ELECTROPHORESIS, 1999; 20: 727-31.

Ruiz-Nuño A, Mayorgas I, Hernández-Guijo JM, Olivares R, Garc1, x, a AG, Gand1, x, a L. Antimigraine dotarizine blocks P/Q Ca2+ channels and exocytosis in a voltage-dependent manner in chromaffin cells. European Journal of Pharmacology, 2003; 481: 41-50.

Scepek S, Lindau M. Focal exocytosis by eosinophils--compound exocytosis and cumulative fusion. The EMBO Journal, 1993; 12: 1811-7.

Schroeder TJ, Borges R, Finnegan JM, Pihel K, Amatore C, Wightman RM. Temporally resolved, independent stages of individual exocytotic secretion events. Biophysical Journal, 1996; 70: 1061-8.

Schroeder TJ, Jankowski JA, Kawagoe KT, Wightman RM, Lefrou C, Amatore C. Analysis of diffusional broadening of vesicular packets of catecholamines released from biological cells during exocytosis. Analytical Chemistry, 1992; 64: $3077-83$.

Segura F, Brioso MA, Gómez JF, Machado JD, Borges R. Automatic analysis for amperometrical recordings of exocytosis. Journal of Neuroscience Methods, 2000; 103: 151-6.

Shearer PM. Global seismic event detection using a matched filter on long-period seismograms. Journal of Geophysical Research: Solid Earth, 1994; 99: 13713-25.

Stamoulis C, Chang BS. Application of Matched-Filtering to Extract EEG Features and Decouple Signal Contributions from Multiple Seizure Foci in Brain Malformations. International IEEE/EMBS Conference on Neural Engineering : [proceedings]. International IEEE EMBS Conference on Neural Engineering, 2009; 2009: 514-7. 
Südhof TC. The synaptic vesicle cycle: a cascade of protein-protein interactions. Nature, 1995; 375: 645.

Synaptosoft. MiniAnalysis.

Szymanska AF, Doty M, Scannell KV, Nenadic Z. A supervised multi-sensor matched filter for the detection of extracellular action potentials. 2014 36th Annual International Conference of the IEEE Engineering in Medicine and Biology Society, 2014: 5996-9.

Taraska JW, Perrais D, Ohara-Imaizumi M, Nagamatsu S, Almers W. Secretory granules are recaptured largely intact after stimulated exocytosis in cultured endocrine cells. Proceedings of the National Academy of Sciences, 2003; 100: 2070 .

Thureson-Klein A Fau - Klein RL, Klein Rl Fau - Zhu PC, Zhu PC. Exocytosis from large dense cored vesicles as a mechanism for neuropeptide release in the peripheral and central nervous system.

Tobin V, Leng G, Ludwig M. The involvement of actin, calcium channels and exocytosis proteins in somato-dendritic oxytocin and vasopressin release. Frontiers in Physiology, 2012; 3: 261.

Toonen RFG, Verhage M. Munc18-1 in secretion: lonely Munc joins SNARE team and takes control. Trends in Neurosciences, 2007; 30: 564-72.

Travis ER, Wightman RM. SPATIO-TEMPORAL RESOLUTION OF EXOCYTOSIS FROM INDIVIDUAL CELLS. Annual Review of Biophysics and Biomolecular Structure, 1998; 27: 77-103.

van Kempen GTH, vanderLeest HT, van den Berg RJ, Eilers P, Westerink RHS. Three Distinct Modes of Exocytosis Revealed by Amperometry in Neuroendocrine Cells. Biophysical Journal, 2011; 100: 968-77.

Verhage M, Sørensen Jakob B. Vesicle Docking in Regulated Exocytosis. Traffic, 2008; 9: 1414-24.

Voets T, Neher E, Moser T. Mechanisms Underlying Phasic and Sustained Secretion in Chromaffin Cells from Mouse Adrenal Slices. Neuron; 23: 607-15. 
Walter AM, Pinheiro PS, Verhage M, Sørensen JB. A Sequential Vesicle Pool Model with a Single Release Sensor and a Ca2+-Dependent Priming Catalyst Effectively Explains Ca2+-Dependent Properties of Neurosecretion. PLOS Computational Biology, 2013; 9: e1003362.

Wang C-T, Grishanin R, Earles CA, Chang PY, Thomas FJM, Chapman ER, Jackson MB. Synaptotagmin Modulation of Fusion Pore Kinetics in Regulated Exocytosis of Dense-Core Vesicles. Science, 2001a; 294: 1111-5.

Wang C-T, Lu J-C, Bai J, Chang PY, Martin TFJ, Chapman ER, Jackson MB. Different domains of synaptotagmin control the choice between kiss-and-run and full fusion. Nature, 2003; 424: 943.

Wang J, Chatrathi MP, Tian B. Microseparation Chips for Performing Multienzymatic Dehydrogenase/Oxidase Assays: Simultaneous Electrochemical Measurement of Ethanol and Glucose. Analytical Chemistry, 2001b; 73: 1296300.

Wang J, Pumera M. Dual Conductivity/Amperometric Detection System for Microchip Capillary Electrophoresis. Analytical Chemistry, 2002; 74: 5919-23.

Westerink R, Rook M, Beekwilder J, J Wadman W. Dual role of calbindin-D 28K in vesicular catecholamine release from mouse chromaffin cells, 2006.

Wightman RM. Monitoring Molecules: Insights and Progress. ACS Chemical Neuroscience, 2015; 6: 5-7.

Wightman RM, Jankowski JA, Kennedy RT, Kawagoe KT, Schroeder TJ, Leszczyszyn DJ, Near JA, Diliberto EJ, Viveros OH. Temporally resolved catecholamine spikes correspond to single vesicle release from individual chromaffin cells. Proceedings of the National Academy of Sciences of the United States of America, 1991; 88: 10754-8.

Winkler H, Sietzen M, Schober M. The Life Cycle of Catecholamine - storing Vesiclesa. Annals of the New York Academy of Sciences, 2006; 493: 3-19.

Yang Y, Craig TJ, Chen X, Ciufo LF, Takahashi M, Morgan A, Gillis KD. Phosphomimetic Mutation of Ser-187 of SNAP-25 Increases both Syntaxin Binding and Highly $\mathrm{Ca}(2+)$-sensitive Exocytosis. The Journal of General Physiology, 2007; 129: 233-44. 
Zachek MK, Hermans A, Wightman RM, McCarty GS. Electrochemical Dopamine Detection: Comparing Gold and Carbon Fiber Microelectrodes using Background Subtracted Fast Scan Cyclic Voltammetry. Journal of electroanalytical chemistry (Lausanne, Switzerland), 2008; 614: 113-20.

Zhao Y, Fang Q, Herbst AD, Berberian KN, Almers W, Lindau M. Rapid structural change in synaptosomal-associated protein 25 (SNAP25) precedes the fusion of single vesicles with the plasma membrane in live chromaffin cells. Proceedings of the National Academy of Sciences, 2013; 110: 14249-54.

Zhou Q, Lai Y, Bacaj T, Zhao M, Lyubimov AY, Uervirojnangkoorn M, Zeldin OB, Brewster AS, Sauter NK, Cohen AE, Soltis SM, Alonso-Mori R, Chollet M, Lemke HT, Pfuetzner RA, Choi UB, Weis WI, Diao J, Südhof TC, Brunger AT. Architecture of the synaptotagmin-SNARE machinery for neuronal exocytosis. Nature, 2015; 525: 62. 


\section{VITA}

Supriya Balaji Ramachandran received a Bachelor's degree in Electrical and

Electronics Engineering from Anna University, India. She then worked for "Tata

Consultancy Services Ltd." and "Teach for India" before starting graduate school. 